\title{
AUTOMATIC DETECTION OF PERIODIC LIMB MOVEMENTS IN SLEEP (PLMS), AND ANALYSIS OF THEIR RELATIONSHIP TO HEART RATE SIGNALS
}

by

\author{
Sharadha Kolappan \\ B.Eng., Ryerson University, 2016 \\ B.Sc., University of Toronto, 2011
}

A thesis

presented to Ryerson University

in partial fulfillment of the requirements for the degree of Master of Applied Science in the program of

Biomedical Engineering

Toronto, Ontario, Canada, 2019

(C) Sharadha Kolappan, 2019 


\section{Author's Declaration}

I hereby declare that I am the sole author of this thesis. This is a true copy of the thesis, including any required final revisions, as accepted by my examiners.

I authorize Ryerson University to lend this thesis to other institutions or individuals for the purpose of scholarly research.

I further authorize Ryerson University to reproduce this thesis by photocopying or by other means, in total or in part, at the request of other institutions or individuals for the purpose of scholarly research.

I understand that my thesis may be made electronically available to the public. 


\begin{abstract}
AUTOMATIC DETECTION OF PERIODIC LIMB MOVEMENTS IN SLEEP (PLMS), AND ANALYSIS OF THEIR RELATIONSHIP TO HEART RATE SIGNALS
\end{abstract}

\author{
Sharadha Kolappan, 2019 \\ Master of Applied Science \\ Biomedical Engineering \\ Ryerson University
}

Periodic Limb Movement in Sleep (PLMS) are a sleep-related disorder of the limbs that increasingly more research has begun to associate with severe Cardiovascular Diseases (CVD). With that said, Polysomnography (PSG), followed by manual scoring, is the conventional approach being used to monitor the disorder. However, patient inconvenience, and the high costs associated with PSG, has probed the need for alternative screening tools to be developed. Moreover, due to the cumbersome and time-consuming nature of manually scoring for PLMS, more studies have begun to look into automated means of detecting PLMS. Hence, while one of the goals of the current thesis was to use the latest clinical specifications to develop an automated Periodic Limb Movement (PLM) detector, the other goal was to look into alternative signals to monitor PLMS.

With that said, in the current thesis, an automated PLM detector was developed and tested on two datasets. In fact, the results were promising in that, correlation coefficients of 0.78 and 0.8 , and absolute differences not greater than 9 and 6 (not including the extreme outliers) respectively, were found when comparing the clinical PLM scores with that of the automated algorithm's PLM scores. Moreover, not only did the automated PLM detector compute PLM scores, it also provided us with PLM segmentation information, i.e., localization of PLM with respect to time.

On the other hand, with regards to finding alternative signals to monitor PLMS, the etiology of PLMS was used in order to validate the use of relatively easily acquirable signals, such as Heart Rate (HR) signals, to monitor the condition. Moreover, core features were extracted from the HR signals and the PLM segmentation information from the developed PLM detector was used in order to perform individuaized classification between PLM and non-PLM segments (per subject). Although the results were promising in that, the percent of correctly identifying a given segment as PLM or non-PLM, using the HR features, across most of the subjects, i.e., especially those with PLM Index $\geq 15$, were around and well above the $70 \%$ range, due to the possibility of other factors interfering with HR during sleep, a more immediate application of the observed PLMS vs HR distinction was, to be able to monitor the autonomic health of an individual, given their PLM information. Specifically, the latter was anticipated to be useful for studies looking into the relationship between PLMS and HR, and thus CVD, or more significantly, those looking into preventing CVD by treating PLM. 


\section{Acknowledgment}

First and foremost, I would like to thank Dr. Sri Krishnan for not only providing me with guidance throughout my research, but also for being an inspirational role model that I highly look up to.

Also, I would like to thank my fellow colleagues from the Signal Analysis Research (SAR) group for their company and valuable feedback throughout my time in the lab: Yashodhan Athavale, Abdelrahman Abdou, Giordano Arezza, Dharmendra Gurve, Sourav Mukhopadhyay, Michael Nigro, Jeevan Pant, Fayez Qureshi, Alice Rueda, Wenjun Yang, and Michael Zara.

I would also like to take this opportunity to thank my professors Dr. Eric Harley, Dr. Kristiina Mai, and Dr. Karthi Umapathy, for guiding me through my courses at Ryerson University.

Last but not least, I would like to thank my parents and brother for being a huge backbone of support, without whom, I wouldn't have been able to successfully accomplish my goals. 


\section{Contents}

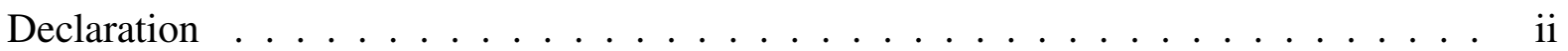

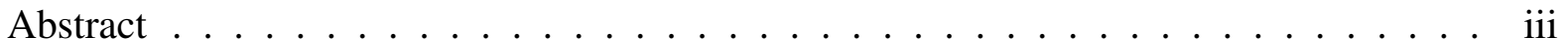

Acknowledgement $\ldots \ldots \ldots \ldots \ldots \ldots \ldots \ldots \ldots$ iv

List of Figures . . . . . . . . . . . . . . . . . . vii

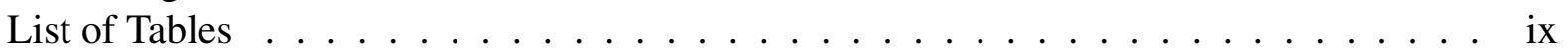

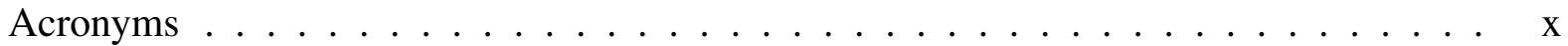

1 Introduction 1

1.1 Motivation . . . . . . . . . . . . . . . . . . . . 1

1.1.1 What are PLMS? . . . . . . . . . . . . . . . . . . 1

1.1.2 Why is it important to detect PLMS? . . . . . . . . . . . . . . 1

1.1.3 How are PLMS currently detected? . . . . . . . . . . . . . . . 2

1.1.4 Issues associated with the current PLMS detection method . . . . . . . 2

1.2 Objectives and Organization of thesis $\ldots \ldots \ldots \ldots$

2 Background $7 \ldots$

2.1 The Autonomic System . . . . . . . . . . . . . . . . . . . . 7

2.2 Etiology of PLMS . . . . . . . . . . . . . . . . . . . . . . . 12

2.3 PLM - HRV Relationship . . . . . . . . . . . . . . . . . . . . . 17

2.4 Summary . . . . . . . . . . . . . . . . . . . . . . . . 18

3 Automated PLM Identification and Scoring 19

3.1 Literature Survey . . . . . . . . . . . . . . . . . . . . . . . . . . . . 19

3.2 Method - Pre-processing, PLM Identification, Segmentation and scoring . . . . . 23

3.2 .1 EMG pre-processing . . . . . . . . . . . . . . . . 23

3.2.2 LM (Leg Movement) Activity Identification . . . . . . . . . . . . . . . 23

3.2.3 Mono-lateral PLM Identification and Segmentation . . . . . . . . . . . . 27

3.2.4 Bi-lateral PLM Identification and Segmentation . . . . . . . . . . . . . . 30

3.3 Results and Discussion - Analysis . . . . . . . . . . . . . . 33

3.3.1 Comparison of Clinical vs. Algorithm Scores . . . . . . . . . . . . . 33

3.3.2 Analysis of PLM vs. Sleep Stages . . . . . . . . . . . . . . . . . 45

3.3.3 Analysis of LM and IMI Duration of PLM per Sleep Stage . . . . . . . . . 49

3.4 Summary . . . . . . . . . . . . . . . . . . . . . . . . 59 
4 Analysis of PLMS in relation to HR Signals $\quad 60$

$4.1 \quad$ ECG Pre-processing . . . . . . . . . . . . . . . . . . . . . 61

4.1.1 R Peak detection and HR Signal acquisition . . . . . . . . . . . . . . . 61

4.1.2 ECG Signal shortcomings . . . . . . . . . . . . . . . . . . . 65

4.1.3 Overcoming the shortcomings associated with ECG Signals . . . . . . 67

4.2 Feature Extraction and PLMS Classification using HR Signals . . . . . . . . . 70

4.2.1 Extracting core features from HR signals . . . . . . . . . . . . 70

4.2.2 Individualized 'PLM and Sleep Labelling' of data . . . . . . . . . . . . . 73

4.2.3 Classifier and Performance metrics _. . . . . . . . . . . . . . 80

4.2.4 Classification results on Normal (PLM Index $<15$ ) and Abnormal groups (PLM Index $\geq 15$ ) across the 3 scenarios $\ldots \ldots \ldots$. . . . . . . 84

4.3 Summary . . . . . . . . . . . . . . . . . . . . . . . . . 95

5 Conclusion $\quad 96$

5.1 Summary, Limitations, and Future Work . . . . . . . . . . . . . . . . . 96

5.2 Conference Proceeding from the thesis . . . . . . . . . . . . . . 101

$\begin{array}{ll}\text { Bibliography } & 102\end{array}$ 


\section{List of Figures}

1.1 Organisation of the Thesis $\ldots \ldots \ldots \ldots \ldots \ldots$

2.1 Sympathetic Innervation, by BruceBlaus (2014) [14], with the connections to the heart highlighted. . . . . . . . . . . . . . . . . . . . 9

2.2 Parasympathetic Innervation, by BruceBlaus (2014) [14], with the connections to the heart highlighted. . . . . . . . . . . . . . . . . . . . 10

2.3 The sequence of events that are thought to be involved in the PLM mechanism. Re-drawn based on [15] (permission granted) . . . . . . . . . . . . . 13

3.1 Developed PLM Detection Algorithm based on [7] $\ldots \ldots \ldots 22$

3.2 EMG pre-processing steps on a 5 min EMG segment from a subject . . . . . . . 24

3.3 Baseline and LM Onset/Offset thresholds on a 5 min EMG segment from a subject 25

3.4 Implementation of Ferri et al.'s [8] PLM detection process on a 5 min EMG segment from a subject . . . . . . . . . . . . . . . . 26

3.5 PLM identified on a 5 min EMG segment from a subject . . . . . . . . . . . . 27

3.6 Example of one and less than half a portion of a PLM segment taken from a subject - Implementation of PLM specifications summarized by [7] for mono-lateral limbs 29

3.7 Example of 2 LM from opposite limbs from a subject - Implementation of PLM specification summarized by $[7]$ for bi-lateral limbs . . . . . . . . . . . . . 32

3.8 Occurrence of PLM segments identified mono-laterally and bi-laterally for a subject during one PSG session. . . . . . . . . . . . . . . . . 33

3.9 Dataset A - Clinical vs. Algorithm scores for 28 (out of 56) subjects (AHI $\geq 5$ ) . . 36

3.10 Dataset B - Clinical vs. Algorithm scores for 11 (out of 38) subjects (AHI $\geq 5$ ) . . 37

3.11 Dataset A - Clinical vs. Algorithm scores for 28 (out of 56) subjects (AHI $<5$ ) . . 39

3.12 Dataset B - Clinical vs. Algorithm scores for 27 (out of 38) subjects (AHI $<5$ ) . . 40

3.13 Dataset B - Clinical vs. Algorithm scores for 25 (out of 38) subjects $(\mathrm{AHI}<5$ ) not including scores from 2 subjects (outliers) . . . . . . . . . . . . . . . 41

3.14 Dataset A - Bland Altman plot for 28 (out of 56) subjects $(\mathrm{AHI}<5) \ldots \ldots$

3.15 Dataset B - Bland Altman plot for 27 (out of 38) subjects $(\mathrm{AHI}<5) \ldots 45$

3.16 Dataset B - Bland Altman plot for 25 (out of 38) subjects $(\mathrm{AHI}<5$ ) not including scores from 2 subjects (outliers) . . . . . . . . . . . . . . . . 46

3.17 Dataset A - PLM Incidence with respect to the different sleep states . . . . . . . 4 48

3.18 Dataset B - PLM Incidence with respect to the different sleep states . . . . . . . . 49 
3.19 Dataset A - LM duration distribution of PLM occurring during the awake state . . 51

3.20 Dataset B - LM duration distribution of PLM occurring during the awake state . . 52

3.21 Dataset A - LM duration distribution of PLM occurring during other sleep states . 53

3.22 Dataset B - LM duration distribution of PLM occurring during other sleep states . 54

3.23 Dataset A - IMI duration distribution of PLM occurring during the awake state . . 55

3.24 Dataset B - IMI duration distribution of PLM occurring during the awake state . . 55

3.25 Dataset A - IMI duration distribution of PLM occurring during other sleep states . 57

3.26 Dataset B - IMI duration distribution of PLM occurring during other sleep states . 58

4.1 Overview of Chapter $4 \ldots \ldots \ldots$. . . . . . . . . . . . . . 62

4.2 Raw ECG shown. R peaks detected using Pan Tompkins. . . . . . . . . . . . . . 63

4.3 HR - corresponding to a longer $(5 \mathrm{~min}$ ) portion of the ECG signal shown in Figure $4.2 \ldots \ldots \ldots \ldots \ldots \ldots \ldots$

4.4 Ectopic beats picked up along with QRS resulting in occasional high HR. . . . . . 64

4.5 HR - corresponding to a longer (5 min) portion of the ECG signal shown in Figure

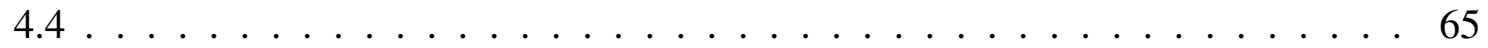

4.6 ECG - Long T wave along with QRS detected as QRS using Pan Tompkins. . . . . 66

4.7 HR - corresponding to a longer $(5 \mathrm{~min}$ ) portion of the ECG signal shown in Figure

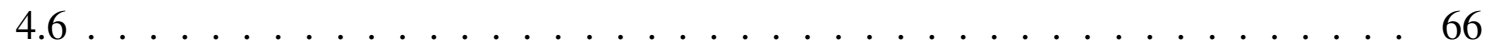

4.8 ECG - Possible movement noise erroneously picked up as QRS using Pan Tompkins. 67

4.9 HR - corresponding to a longer $(5 \mathrm{~min})$ portion of the ECG signal shown in Figure

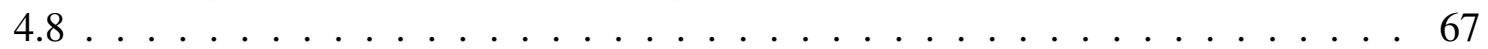

4.10 Possible instrumentation noise erroneously picked up as QRS using Pan Tompkins. 68

4.11 Example of a clean HR signal obtained from the entire duration of a PSG session . 69

4.12 Example of a non-clean HR signal obtained from the entire duration of a PSG session 70

4.13 Implementation of HT on a sample HR signal . . . . . . . . . . . . . . . . 72

4.14 The core features, i.e., LFia $a_{\text {median }}$ and HFia $a_{\text {median }}$, extracted from a sample HR signal, obtained from the entire duration of a PSG session . . . . . . . . . . 73

4.15 The application of normal and abnormal (PLM) labels across the core features extracted from a sample HR signal, obtained from the entire duration of a PSG

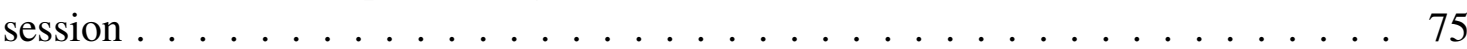

4.16 The application of sleep weightage across the core features extracted from a sample HR signal, obtained from the entire duration of a PSG session $\ldots \ldots$. . . . . 76

4.17 Sample Abnormal Subject $(\mathrm{PLM} \geq 15) \ldots \ldots \ldots$

4.18 Sample Normal Subject $(\mathrm{PLM}<15) \ldots \ldots$. . . . . . . . . . . 79

4.19 Implementation of bagged tree classifier on an Abnormal Subject . . . . . . . . . 82

4.20 Dataset A - Classification Results on Normal and Abnormal groups of Subjects . . 86

4.21 Dataset B - Classification Results on Normal and Abnormal groups of Subjects . 87

4.22 Sample 'Normal' Subject with Sleep Apnea (and Respiratory-related PLM) . . . . 94 


\section{List of Tables}

3.1 Recent studies automating PLM detection using software . . . . . . . . . . . 20

3.2 Demographics of Dataset $\mathrm{A}: \mathrm{N}=56(\mathrm{M}=32$ and $\mathrm{F}=24) \ldots \ldots$

3.3 Demographics of Dataset B: $\mathrm{N}=38(\mathrm{M}=20, \mathrm{~F}=17$, Undefined $=1) \quad \ldots 35$

4.1 MCC Scores of Dataset A for the 2 Groups of Subjects across the 3 Case Scenarios 88

4.2 MCC Scores of Dataset B for the 2 Groups of Subjects across the 3 Case Scenarios 89 


\section{Acronyms}

AHI Apnea-Hypopnea Index.

ANS Autonomic Nervous System.

bCLM bi-lateral Candidate Limb Movement.

CA Classification Accuracy.

CLM Candidate Limb Movement.

CNS Central Nervous System.

CVD Cardiovascular Diseases.

DWT Discrete Wavelet Transform.

ECG Electrocardiography.

EEG Electroencephalography.

EMG Electromyography.

EOG Electrooculography.

HR Heart Rate.

HT Hilbert Transform.

IMI Inter-Movement Interval.

IML Intermediolateral.

LM Leg Movement.

MCC Matthews Correlation Coefficient. 
mCLM mono-lateral Candidate Limb Movement.

PLM Periodic Limb Movement.

PLMD Periodic Limb Movement Disorder.

PLMI Periodic Limb Movement Index.

PLMS Periodic Limb Movement in Sleep.

PLMW Periodic Limb Movement during Wake.

PNS Peripheral Nervous System.

PSG Polysomnography.

RBD REM Sleep Behavior Disorder.

RLS Restless Leg Syndrome.

SA Sleep Apnea.

SAN Sino-Atrial Node.

SDI Sleep Disorder Index.

SoNS Somatic Nervous System. 


\section{Chapter 1}

\section{Introduction}

\subsection{Motivation}

\subsubsection{What are PLMS?}

PLMS, predominantly found in the aging population, are a common sleep-related movement disorder that is, more often than not, associated with the lower limbs of the body [1]. According to the International Classification of Sleep Disorders - Third Edition (ICD-3), by the American Academy of Sleep Medicine [1], PLMS involves "periodic episodes of repetitive limb movements that occurs during sleep" [1]. In fact, PLMS typically occur involuntarily such that, individuals experiencing it are normally not aware of it [1].

\subsubsection{Why is it important to detect PLMS?}

That being said, as reviewed in [2], [3], [4], more research is beginning to relate PLMS with the development of stroke and heart diseases. One of the dominant theories behind the latter is that the incidence of PLMS, over prolonged periods of time, could lead to the development of cardiovascular conditions such as stroke. This, is supposedly, due to a heightened effect of the sympathetic system commonly found to be associated with either of the conditions [2], [3], [4]. Nonetheless,

given that stroke and heart diseases themselves are some of the leading causes of death in the world, 
i.e., both of them globally accounting for as much as 15 million deaths in the year 2015 alone [5], contemplating more on a sleep-related movement disorder such as PLMS could be quite impactful.

\subsubsection{How are PLMS currently detected?}

At present, the gold standard test for detecting PLMS, or any other sleep disorder for that matter, is a sleep study test [6]. The former is more formally known as PSG [6]. Electromyography (EMG), Electrocardiography (ECG), Electroencephalography (EEG), and Electrooculography (EOG) are some of the main parameters typically acquired during a sleep study test. In essence, PSG obtains signals from various parts of the body, namely, the limbs, heart, brain, and eyes respectively [6]. Of relevance here, it is of interest to mention that it is the EMG portion of PSG, i.e., the EMG measurements obtained from the tibialis anterior leg muscles in particular, that is primarily used in order to detect the limb movements associated with PLMS [1]. Specifically, a person is diagnosed with PLMS if he or she exhibits a sequence of at least four consecutive limb movements of specific durations, each occurring at specific intervals of time [7]. In fact, an index is further used to indicate the severity, hence abnormal levels of PLMS (incidence), in a person. These are discussed in much detail in the upcoming chapters, i.e., chapter 3 to be exact.

\subsubsection{Issues associated with the current PLMS detection method}

PSG, as an overall diagnostic tool, has certain practical limitations associated with it. To start with, aside from the measurements mentioned earlier, PSG involves the measurement of other param-

eters such as oxygen saturation levels, incidence of snoring, and thoracic movements [6]. As a result, one common drawback related with PSG is patient discomfort [2]. In other words, from a convenience standpoint alone, the instrumentation associated with making the various measurements on a given individual reduces the applicability of using PSG as a whole or, decreases the ability of obtaining parameters reflecting the 'true' sleep patterns of the individual being analyzed.

That being said, not only is a trained technician needed to mount the various electrodes for 
making the various measurements, but a trained technician is also needed to detect and score, as dictated by established standards, the various sleep events occurring during an individual's sleep session. In fact, implementing the latter could be a tedious and time-consuming task. For example, one can only imagine the effort and time that a sleep technician would have to endure to detect a phenomenon like PLMS alone, for typically 6 to 8 hours of data per individual.

Along the same lines, another major drawback associated with a sleep study test is, the expense associated with it [8]. For example, even in a developed country like Canada, i.e., within the province of Ontario specifically, a sleep study test can cost around $\$ 750$ at the very least [8]. In fact, at some sleep study centers, prices can even go as high as 1900 Canadian dollars [8]. Thus, clearly the expense associated with PSG is yet another factor that could affect its applicability as a primary tool for sleep disorder diagnosis.

Thus, as a whole, using PSG, for all of the mentioned reasons, to detect PLMS in this case, is not a convenient and cost-effective option. Note that, given that PLMS have been associated with cardiovascular diseases (for e.g., [4]), monitoring (and hindering) their progression over time for instance, is a significant topic at hand. However, for the reasons mentioned above, using PSG for the long-term monitoring (and management) of PLMS in particular, is less ideal.

\subsection{Objectives and Organization of thesis}

All that being said, shown in Figure 1.1 is the outline of the organization of the current thesis.

To start with, it is of interest, in the current thesis, to be able to find alternative signals, i.e., other than the EMG from PSG for example, to detect PLMS. By doing so, the idea here was to be able to alleviate the need to be wired uncomfortably to so many instruments just to monitor the condition. Specifically, it is expected that the latter would surpass the need for an affected individual especially, to undergo a full-blown sleep study test in subsequent sessions to monitor the progression (or management) of the condition in a long-term setting. That being said, in the current thesis, the etiology of PLMS was used in order to validate the choice of the alternative signal chosen to be used to monitor PLMS. In this case, HR signals, obtained from ECG, which is an easily attainable 


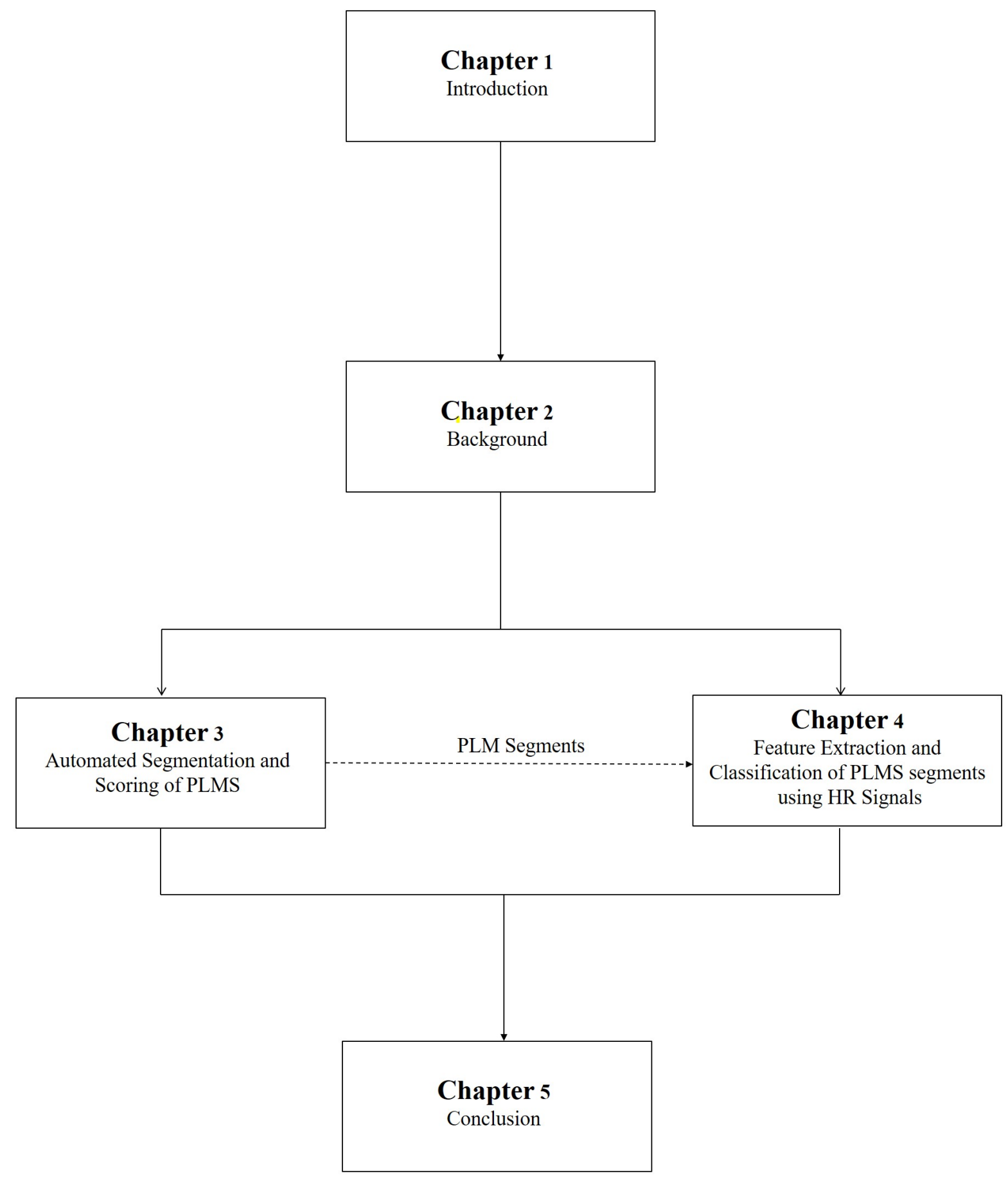

Figure 1.1: Organisation of the Thesis 
signal (through ECG patches [9] for example), is explored in depth in chapter 2. Specifically, the relationship of the HR signal with respect to PLMS is explored from a physiological standpoint. Additionally, a brief literature survey conducted on studies analyzing the relationship of the two, from an empirical standpoint, is provided.

On a different note, in order to alleviate the time-consuming and cumbersome nature of detecting and scoring for PLMS, one of the objectives of the current thesis was to be able to automate the detection of PLMS from PSG. Note though, as would be briefly reviewed later, that attempts to automate the detection of PLMS have already been, and continues to be made. However, given that visual and manual scoring still remains to be the gold standard method enforced in many hospitals for detecting PLM [2], a step-by-step attempt at effectively automating the detection of PLMS, based on latest standards [7], is shown here. Specifically, the latter is provided in chapter 3 of the current thesis. In fact, the performance of the developed software tool on actual datasets is also provided (in chapter 3). Notwithstanding, using the PLM detected using the developed algorithm, frequency distributions of the incidence of PLM across the different stages of sleep, and changes in their characteristics, i.e., like Leg Movement (LM) duration for example, across the different sleep stages, etc., are additionally provided. Specifically, while the former is expected to affirm the sleep stage in which we are more likely to be able to detect PLM, the latter is expected to provide us with some much needed empirical insights into the changes in the characteristics of PLM across the different sleep stages.

All that being said, in order to demonstrate the possibility of monitoring PLMS using alternative signals, the knowledge of the occurrences of PLM across the PSG session (obtained from chapter 3) per subject was used. Specifically, the latter was used along with features extracted from the HR signal per subject to map a personalized [10] 'PLM versus non-PLM' feature space for the subject being analyzed. To be exact, the choice of HR features used, the challenges that were encountered when extracting the latter from long-term ECG signals, the steps that were taken to overcome these challenges, and finally, the results form classifying between the PLM and nonPLM regions of a subject using the chosen features from HR signals is discussed in chapter 4 . In essence, the idea here was to be able to explore the possibility of learning from past PSG sessions, 
the feature space occupied by HR features corresponding to PLM and non-PLM, and being able to use the latter to predict whether new instances of HR values extracted from a subject would belong to that of the PLM or non-PLM regions of the subject. Note that, this process is rather unique given that a personalized map of the HR-based features with respect to PLM is created for each subject being tested. Above all, it is expected that the latter would provide one with the means of monitoring PLMS using easily obtainable signals such as HR.

Likewise, by mapping the regions of PLM vs non-PLM regions using the HR signals obtained per subject, it was also of interest in the current study to be able to examine whether, given the PLM information, the HR features closely reflecting the autonomic health of the individual can be monitored over time. In other words, provided that the link between PLM and CVD mentioned earlier exists, perhaps one can monitor the changes in HR parameters in response to treatment of PLM, for example. In fact, as reviewed in (for e.g., [4]), certain studies have attempted to decipher whether treating PLM can directly reduce the risk of CVD, but the results have remained controversial [11], [12]. Hence, the idea here is to be able to provide more viable markers for such studies analyzing the effect of PLM on HR, and hence, on CVD. 


\section{Chapter 2}

\section{Background}

Before discussing the etiology of PLMS and its relationship to HR signals, it is significant here to introduce the autonomic system and the terminology associated with the latter. To start with, the nervous system is made up of the Central Nervous System (CNS) and Peripheral Nervous System (PNS) [13]. While the CNS is made up of the brain and spinal cord, the PNS is comprised of nerves and ganglia (group of cell bodies) that branch out of the CNS. In fact, the PNS can further be divided into the Autonomic Nervous System (ANS) and Somatic Nervous System (SoNS). Briefly, while the SoNS typically exerts both voluntary and involuntary control, i.e., over the skeletal muscles and spinal reflexes in the body, respectively, the ANS is responsible for the involuntary control of the viscera of the body [13]. That being said, of relevance here, described in detail below (in Section 2.1), is the ANS.

\subsection{The Autonomic System}

The ANS [13] is mainly comprised of three divisions, namely, the sympathetic, parasympathetic, and enteric divisions. Since the enteric division is predominantly involved with the control of the digestive processes in the body, in the current thesis, it is not delved into any further. That being said, the other two divisions, i.e., the sympathetic and parasympathetic divisions, are commonly referred to as the 'fight-or-flight' and 'rest and digest' system, respectively. As the names suggest, 
while the former typically prepares the body for an imminent threat, the latter is predominant during rest [13]. Shown in Figure 2.1 and Figure 2.2 respectively, are the innervations (illustrated using [14]) associated with the sympathetic and parasympathetic systems in relation to the central nervous system, i.e., the brain and the spinal cord. Note how, as can be inferred from the figure, numerous organs can simultaneously be activated or, de-activated, by both the systems, depending on the situation at hand. Moreover, for most cases, the effect of either of the systems on the body are opposite to each other, i.e., such that, taking the example of the effect of either of the systems on the heart for example, while one system increases the HR, the other decreases the HR. However, this is not necessarily always the case. For instance, with respect to the salivary glands for example, the result of both the sympathetic and parasympathetic systems is to increase salivary production [13].

Nevertheless, in order to better understand the mechanism of how the ANS functions, an example of the cascade of bodily events that take place in a fear-evoking situation, such as that evoked when encountering a bear for example, is described. Typically, as hinted above, it is the sympathetic branch of the ANS that is activated upon encountering a fear-evoking situation. In essence, in order to prepare the body to run away from the situation, or stay and fight the fearful encounter, certain bodily events would occur. For instance, an increased HR, an increased respiration rate, an inhibition of the digestive system, an inhibition of the reproductive system, etc., are just a few examples of the bodily changes that take place [13] in response to the fear-evoking situation which, in this case, is the encounter with the bear. In short, the idea here would be to shut down the organs and systems that are not important at that instant in time, but rather, to use the bodily resources efficiently, i.e., by turning on the organs and systems that could be helpful for the individual to handle the situation at hand.

That being said, as mentioned earlier, not all the organs are activated by the ANS at all times. In other words, the tissues or organs stimulated or inhibited at any given moment depends on the stimulus at hand. For example, a scenario in which not all the organs are necessarily stimulated is when the body is trying to maintain homeostasis, i.e., a condition of 'stability' in the body [13]. For example, if at any given moment, high blood pressure is sensed in the walls of the aorta for example 


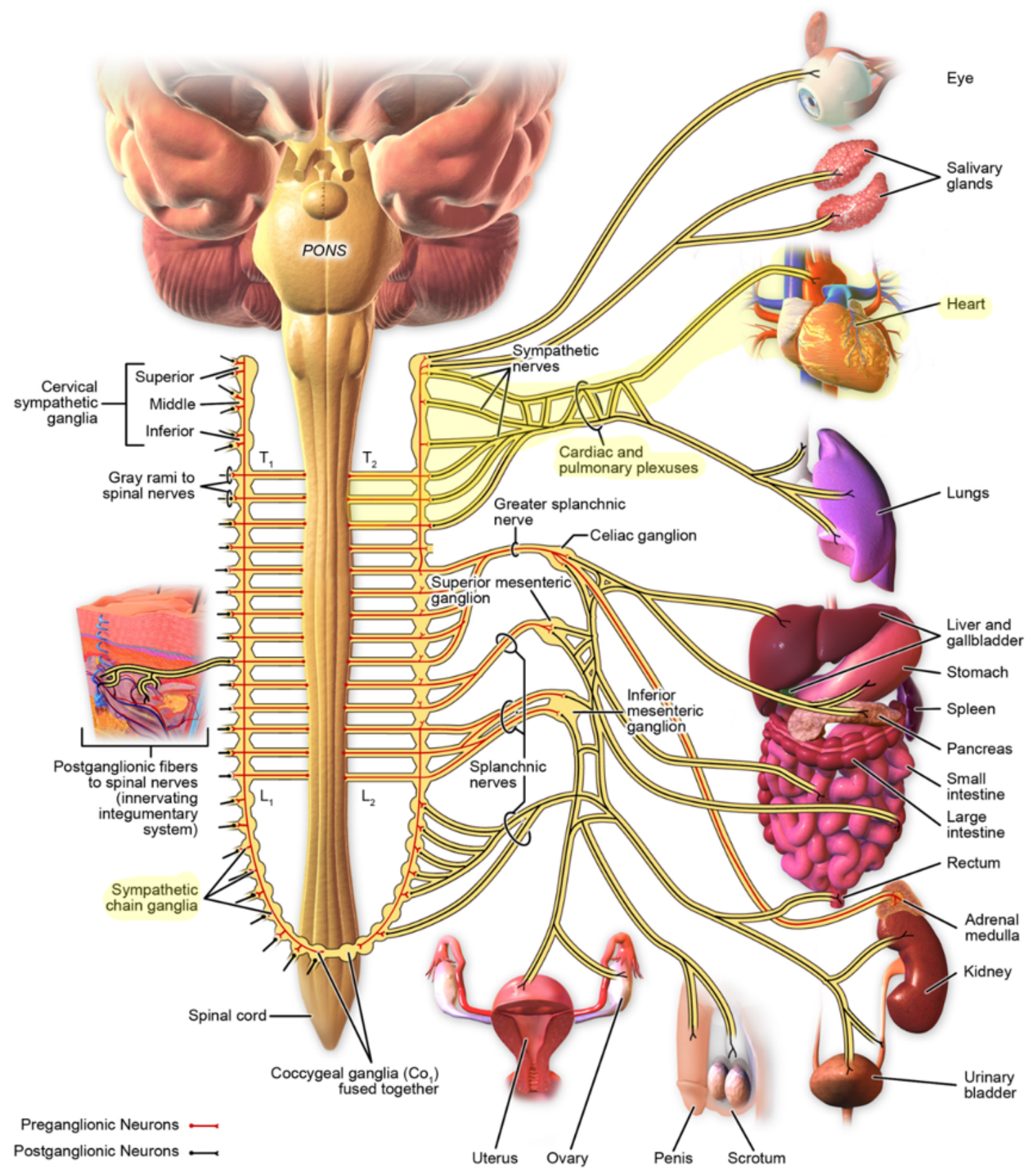

Figure 2.1: Sympathetic Innervation, by BruceBlaus (2014) [14], with the connections to the heart highlighted. 


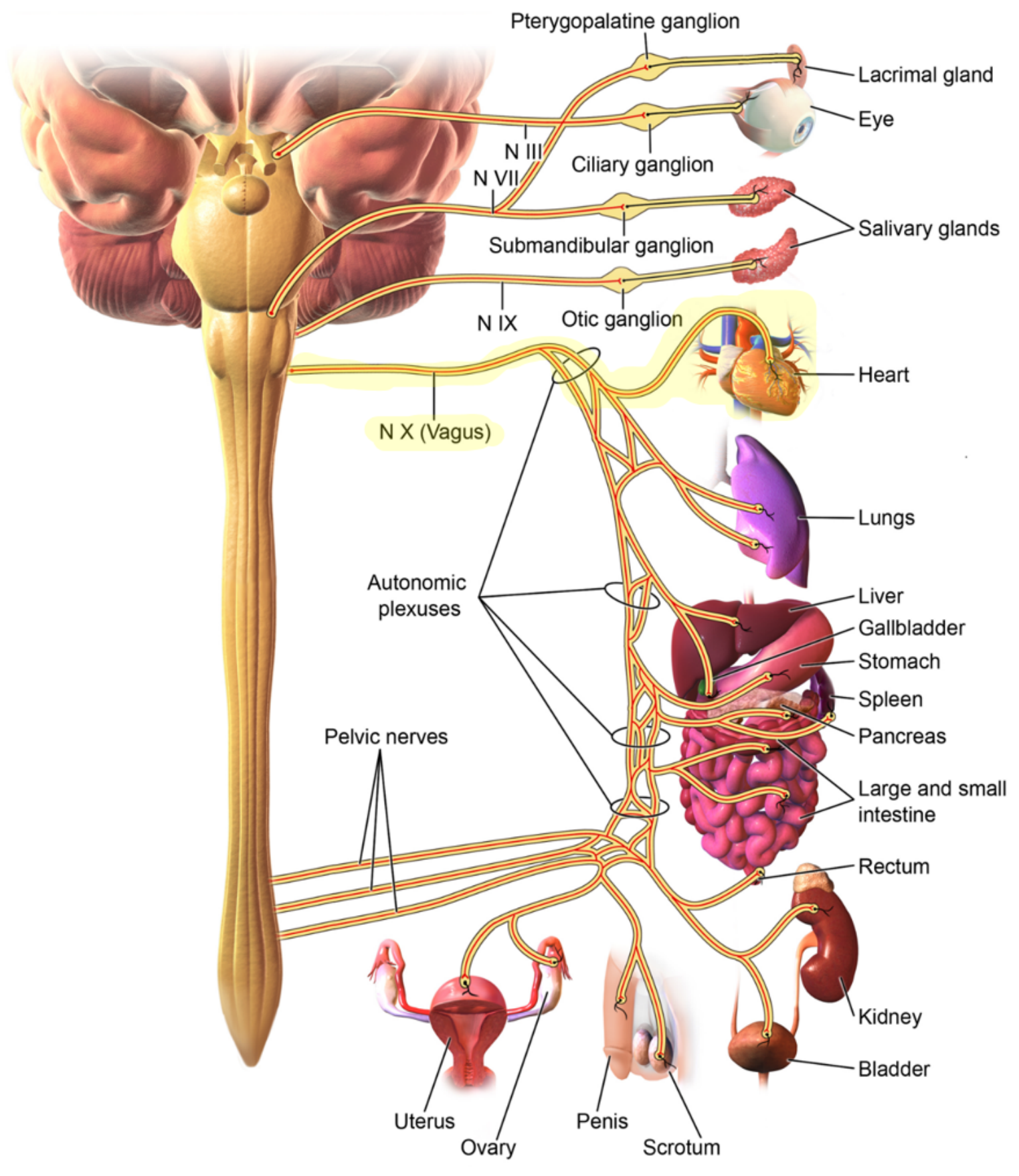

Figure 2.2: Parasympathetic Innervation, by BruceBlaus (2014) [14], with the connections to the heart highlighted. 
(by cells referred to as baroreceptors), the body would respond by activating the parasympathetic branch (in a reflex fashion) to help lower the blood pressure. In this case, blood vessels would dilate, and HR would decrease for example [13], to decrease the blood pressure. Essentially, the latter only involves the activation or de-activation of a select set of tissues or organs that are directly involved in the process of decreasing the blood pressure.

With that said, the two examples described above demonstrate how, different tissues, organs, or systems in the body, are activated, or repressed, by the different branches of the ANS, at single, or multi-faceted levels, at any given time, in response to a stimulus. Notwithstanding, there may also exist other situations in the body in which the ANS may not necessarily be activated in response to a stimulus, but rather, be accidently activated or de-activated due to the insufficient production of hormones, severed nerve connections in the body, etc., to name a few [13]. In fact, one of the dominantly proposed theories for the etiology of PLMS, which is discussed in depth in Section 2.2 that follows, is that the sympathetic system is activated when it is not supposed to [15]. Moreover, as discussed in Section 2.3, there also exists in the literature, empirical findings that support the latter (for e.g., [16]).

That being said, in order to better understand the anatomical aspects of the etiology of PLMS discussed later, it is of interest to mention here that the sympathetic system, unlike the parasympathetic system (with the exception of a few connections in the parasympathetic system), has a visible 'docking' station in which the nerves branching out from the spinal cord 'docks' at before conveying a signal to the target tissue [13]. Specifically, this is evident in Figure 2.1 in which, the dominantly found 'docking' station, i.e., the sympathetic chain ganglia, is highlighted. Note that, in the upcoming discussions, the latter is also interchangeably referred to as the postganglionics. Nonetheless, the nerves branching out from spinal cord to the sympathetic chain ganglia is referred to as the pre-ganglionic neurons, and the nerves branching out from the sympathetic chain ganglia to the target tissues is referred to as the post-ganglionic neurons [13]. However, this doesn't mean that the parasympathetic system does not have a 'relay' mechanism by which the signals pass from the CNS to the target tissue. In fact, the latter also has pre-ganglionic neurons signaling to the post-ganglionic neurons to convey information to the target tissue, but unlike that of sympathetic 
system, the post-ganglionic neurons of the parasympathetic system generally tend to be shorter, and moreover, the 'relay' station, with the exception of a few nerves, tend to be closer to the target organ [13]. To put things into perspective, taking the example of the connections to the heart for example, while the sympathetic system connects to the heart via short preganglionic nerves that relays signals through the long post-ganglionic nerves enclosed within the cardiac and pulmonary 'plexus' (plexus being a term that is used to refer to a network of nerves [13]), the parasympathetic system connects to the heart via a long pre-ganglionic nerve referred to as the Vagus nerve, that relays signals through the short post-ganglionic neurons found closer to the heart cells (pacemaker cells) [13]. Of interest here, these connections and structures are highlighted in the Figures 2.1 and 2.2, depicted earlier. All that being said, now that a better understanding of the autonomic system is obtained, the etiology of PLMS, which has been proposed to have some association to the sympathetic nervous system [15], is discussed in the section that follows.

\subsection{Etiology of PLMS}

Illustrated in Figure 2.3, is the sequence of events that are likely to occur in an individual affected with PLMS. From our understanding, the latter is one of the most dominant and explicit theories that exist in the literature for the etiology of PLMS [15]. In fact, controversies exist regarding the pathophysiology of PLMS, and according to [17], the latter is still "an open issue". However, since the theory provided in [15] is in line with most of the theories proposed for the pathophysiology of PLM, i.e., specifically those associated with the inhibition of the reflexes at the spinal cord level, or dopamine depletion at the CNS level, or simply, "PLMS being the effect of a hyperactive sympathetic system" [17], it is hoped that, by delving further in depth into the mechanism proposed by [15], that a wholesome understanding of the PLM etiology could be achieved. That being said, listed below, from A. to G., as well as depicted in Figure 2.3, are the sequence of events that are proposed to be involved in the PLM mechanism [15]: 


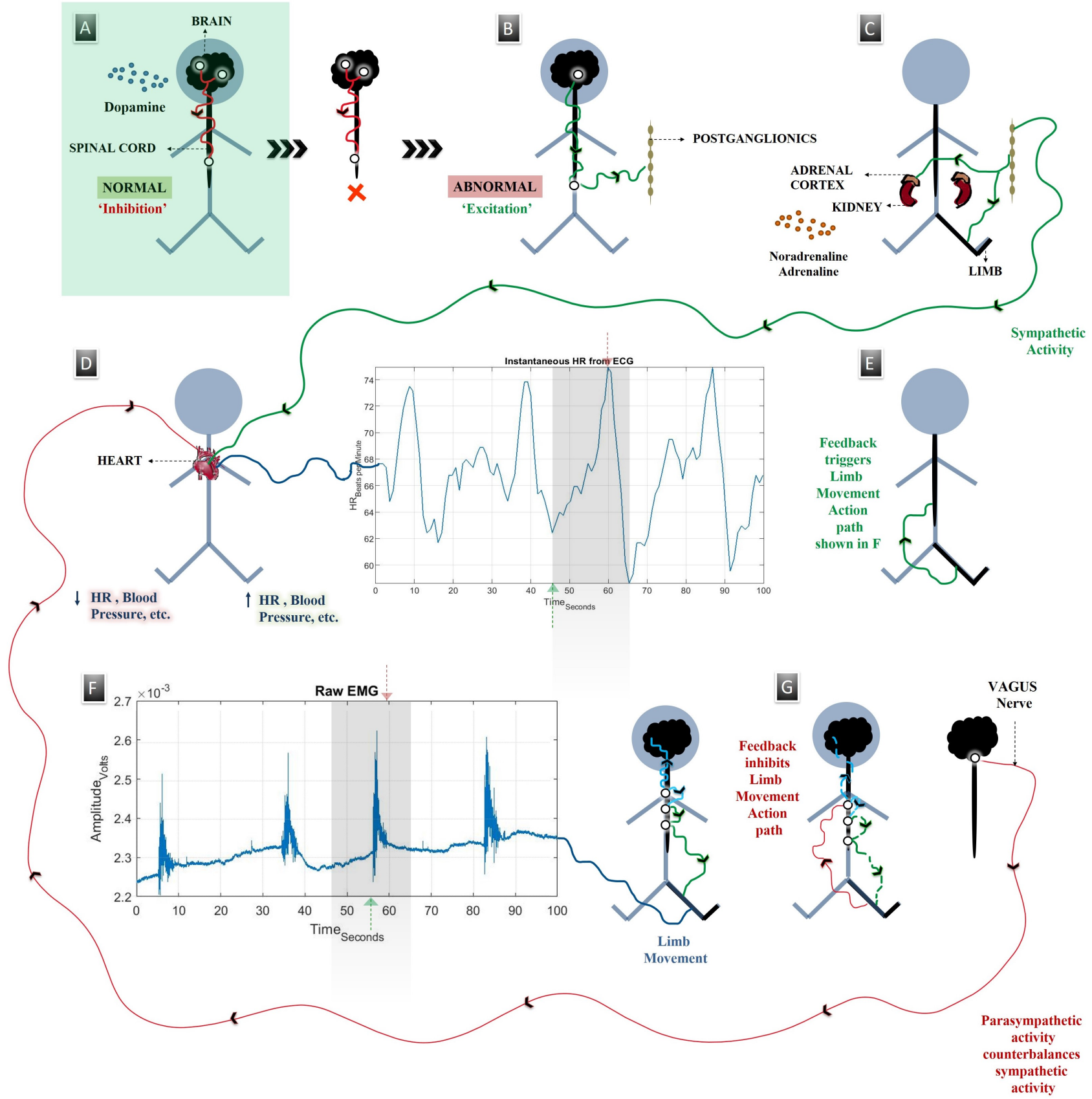

Figure 2.3: The sequence of events that are thought to be involved in the PLM mechanism. Re-drawn based on [15] (permission granted) 
A. Note that, a common proposition, based off of select lesion-specific or knock-out gene (animal) studies [18] is that, the inhibition of the signaling path by a group of neurons collectively belonging to the 'A11' group of cells in the brain leading up to the 'dorsal raphe' group of cell bodies present at the brain level, as well as (mainly) the 'Intermediolateral (IML)' group of cell bodies present at the spinal cord level, does not function as required. This could perhaps be due to insufficient amounts of neurotransmitters involved at the origin of the path like Dopamine, or degeneration of the path itself, etc. [15]. Nonetheless, since the abnormal turn of events emerge provided that the two structures mentioned in the signaling path are not inhibited, the normal case scenario, in the current thesis, is referred to as 'NORMAL inhibition'. As a side note, since the goal here is not to delve into the anatomical structures involved in the PLM phenomenon but rather to highlight the HR signals in relation to PLMS, from here onwards, the neuroanatomic structures and nerves involved are not explicitly stated. However, if needed, the exact neuroanatomical circuitry proposed to be involved in the process is provided in [15].

B. That being said, from B. to G., the sequence of events that takes place in the abnormal case scenario that is illustrated. To start with, provided that the inhibition path, for the reasons briefly mentioned above, does not function well, the signals activating the sympathetic nervous system is stimulated. Specifically, for simplicity, this is shown in the figure by the activation of a single pre-ganglionic branch leading up to the sympathetic chain ganglia [15]. As a side note, the latter is referred to here as the 'ABNORMAL excitation' to highlight the fact that the signaling and sequence of the events that follow are not meant to happen in the normal case scenario. Again, given that the primary focus here is rather to decipher the relationship between HR and PLMS, the possibility of the sympathetic system innervating other tissues, such as those associated with narrowing the blood vessels of the body (to increase blood pressure) [19] for example, is not scrutinized here.

C. That being said, the chemicals Adrenaline and Noradrenaline, released from the kidneys, and Noradrenaline from the nerves leading up to the limb muscle respectively, is thought to have a stimulating effect on the limb muscles' afferent nerve endings [15]. Note that, the term afferent 
here collectively refers to the nerves that lead up to the CNS from the target tissues [13]. Also, depending on where it is released, and how it is transmitted, Noradrenaline for example, can can both act as a hormone as well as a neurotransmitter. Nevertheless, from [15], it is inferred that, while the direct sympathetic innervation of the heart leads to an increase in the HR, the release of the mentioned chemicals from the kidney and nerves leading up to the limb muscles is thought to trigger a cascade of signaling events resulting in the eventual movement of the limb. In fact, since Noradrenaline act on the heart cells to increase HR (through postganglionic innervation), the release of the latter from outside (through the kidneys) may also have an enhancing effect on the heart cells to increase HR [13]. That being said, each of the effects, i.e., on the heart and on the limb muscles to be precise, are separately depicted and described in parts D and E that follow. Moreover it is of interest to mention here that, the authors, when referring to the afferent nerve endings, differentiated what is referred to as the high-threshold nerve fibers, from that of the low-threshold nerve fibers [15]. That being said, the latter two are nothing but terminology that is commonly used to refer to nerves that have different stimulus-response characteristics [20].

D. Nevertheless, as already hinted above in C, shown explicitly in this part of the figure (in green), is the sympathetic innervation leading up to the heart that leads to the increase in HR. Specifically, shaded in grey, is one instance of the activation, or, effect of the sympathetic nervous system (i.e., which is depicted in the plot by the increasing trend of HR with respect to time), and one instance of the parasympathetic activity ( i.e., which is depicted in the plot by the decreasing trend of HR with respect to time). That being said, for ease of explanation, from here onwards, only one cycle, i.e., one instance of the activation of the sympathetic system followed by the incidence of a LM, and one instance of the parasympathetic activation, is emphasized. From our understanding, it is anticipated that, the same process takes place for each and all of the LM occurring in a given series of PLMS. Notwithstanding, it is of interest to mention here that, the signals depicted in the HR plot here, and the EMG plot that follows in F, were actual signals taken from a subject affected with PLMS. (The details on the dataset from which the subject was taken from is provided in Chapter 3). 
E. With that said, focusing on the LM-related sequence of signaling events, the stimulation of the so-called high-threshold nerve fibers of the muscles of the leg, that was defined earlier in $\mathrm{C}$, results in feedback signals to be sent back to the CNS [15]. In fact, the sequence of events that follow the latter is shown in $\mathrm{F}$.

F. In particular, it is supposed that, following the feedback signal from the limbs, the signaling path related to the activation of LM, is stimulated [15]. Specifically, by moving the legs in response to the sensations sensed in E (through the high-threshold fibers of the muscles of the leg), the authors stipulated that, through the use of an analogy of 'itch' vs. 'relief', that the process here could be thought of as 'relieving' an 'itch' by scratching the skin. To be clear, the 'relief' here is attained by the activation of LM, and 'itch' is the sensations felt earlier through the high-threshold nerve endings [15]. Regardless, also shown in this part of the figure is the plot of EMG corresponding to the LM activation signal. Note that, the latter is parallel to the HR plot depicted above in D. In essence, the LM shaded in grey here precisely corresponds to the HR increase and decrease, also shown shaded in grey in D. Moreover, as can be deduced from the plots, in general, the action of the sympathetic system precedes the action of the LM from a PLM series. In fact, this is analogous with empirical findings that has found exactly that [21].

G. That being said, as is the case with any excitation that takes place in the human body [13], in an attempt to restore stability, as theorized in [15], it was supposed that, the feedback, this time sensed by the low-threshold nerve fibers from the activated LM, activates, within the CNS (at the spinal cord level), inhibitory paths that inhibit the excitatory path mentioned earlier to be responsible for activating the LM activity [15]. By doing so, it is expected that, the LM in question, comes to a halt [15]. Simultaneously, as marked by the start of the HR decrease in the shaded area of the HR plot, as well as the end of the LM in the EMG plot, it is anticipated that, parasympathetic activation takes over, i.e., wherein, the Vagus nerve in this case, acts to restore the resting HR (by decreasing the elevated HR from before) [13]. In fact, this is explicitly shown (in red) by the parasympathetic innervation connecting back to the heart depicted in D. 
With all that said, as mentioned earlier, it is expected that, this process of activation and inhibition, occurring consecutively with the activation of the sympathetic and parasympathetic system alternates for the subsequent LM taking place in a given PLM series. In essence, what is gathered from this section (through the study of the possible mechanism by which PLM occurs [15]) is that, ECG, or more precisely, the HR signal, given its theorized [15] and observed co-occurrence with PLMS (for e.g., [16]), seems like an apt signal that, in addition to EMG, can be used to monitor PLMS. Likewise, in the case where PLMS and its effect on the autonomic system is to be studied, the background reviewed here provides a theory on how the two are related.

\subsection{PLM - HRV Relationship}

As already mentioned, in support of the physiological standpoint described above, several studies have empirically pointed towards an association between HR and PLM (for e.g., [21]). In particular, by probing into Heart Rate Variability (HRV) [22], i.e., a term collectively used to refer to the analysis of the deviations in the HR signal, many studies have found a significant difference

in the Low Frequency (LF) and High Frequency (HF) components of HRV when comparing the segments of signals corresponding to PLM with that of those that didn't [16], [23], [24]. However, despite the latter, there also exist other kinds of studies, such as those by [25] for example, in which, by analyzing the segments without PLM, i.e., in the individuals diagnosed with and without PLMS, the authors stipulated that, a pathogenesis in the autonomic system could not be associated with PLM since, in their study, they did not find any significant difference between the 'basal' HR (corresponding to the non-PLM segments) of either of the groups of individuals studied. In fact, similar findings were found by [12] as well, in the same context.

That being said though, other than differing with respect to the HR results and segments chosen for comparison, not many of the studies that we came across analyzed the relationship between HR and PLM from the perspective of monitoring one from the other, on a one-to-one basis, especially in the context of long-term signals. In essence, given the differences in the HR parameters, 
depending on whether they came from a region corresponding to PLM or non-PLM, it was of interest, in the current thesis, to keep separate, and hence analyze, all the regions with, and without PLM, within the same individual. This, as mentioned in Chapter 1, is materialized, by localizing the incidence of PLM with respect to time (per individual studied) in Chapter 3, and by comparing the differences in HR parameters with respect to the PLM and non-PLM segments (obtained from Chapter 3) occuring per individual, in Chapter 4.

\subsection{Summary}

In this Chapter, the background on the anatomy and physiology of the ANS, that is more often than not found associated with PLM, was first reviewed. Following the latter, based on some of the dominantly proposed theories, i.e., specifically the ones by [15], the sequence of events thought to be involved in the PLM mechanism was explicitly illustrated. Lastly, empirical findings (for e.g., [16]) that supported the latter, were also identified.

Overall, it was hoped that, from all of the above, the monitoring of PLM from HR, and vice versa, i.e., HR from PLM, were both physiologically, and to some extent, empirically, justified. 


\section{Chapter 3}

\section{Automated PLM Identification and Scoring}

\subsection{Literature Survey}

As discussed in Chapter 1, although PSG followed by manual scoring is the gold standard technique used to detect PLM, many, due to the cumbersome and time-consuming nature of the latter, have started to look into alternative means of detecting PLM. That being said, a couple of studies in recent times that have attempted to look into alternative, or more exactly, automated (over manual) PLM detection methods for example, include, but are not limited to those by [26], [27], [28], [29], and [30]. Specifically, while the primary goal of these studies was to automate the process of PLM detection, others have also attempted to ease the detection process overall by using alternative signals instead, i.e., signals other than those obtained using PSG. Actigraphy [31], for example, is one such signal increasingly being studied. As the latter deserves an analysis of its own, the primary focus here was to only look into those that attempted to automate the process of PLM detection using PSG signals. To start with, it is of interest to note here that such studies attempting to automate the detection process date as far back as the 1990s [32], and have in fact, continued to rise since then [28]. Table 3.1, shown below, illustrates examples of a few recent ones.

Despite attempts by the various studies tabulated below, and many more not discussed here, visual and manual scoring, for the most part, still remains to be the gold standard method enforced in many hospitals for detecting PLM [28]. With that said, it was of interest to provide here yet 
Table 3.1:

Recent studies automating PLM detection using software

\begin{tabular}{|c|c|c|c|}
\hline Authors & $\begin{array}{l}\text { Unique features and/or draw- } \\
\text { backs pertaining to the study }\end{array}$ & $\begin{array}{l}\text { PLM Stan- } \\
\text { dards }\end{array}$ & Dataset \\
\hline $\begin{array}{l}\text { Weinreich et al. } \\
2018[26]\end{array}$ & $\begin{array}{l}\text { Used non-contact sleep- } \\
\text { monitoring device to measure } \\
\text { a novel index, i.e., Sleep } \\
\text { Disorder Index (SDI), } \\
\text { which is the combination } \\
\text { of Periodic Limb Move- } \\
\text { ment Index (PLMI) and } \\
\text { Apnea-Hypopnea Index } \\
\text { (AHI). However, the device } \\
\text { was not able pick up on } \\
\text { limb movements for cer- } \\
\text { tain arrangements of the } \\
\text { device or positionings of the } \\
\text { subject [26]. }\end{array}$ & $\begin{array}{l}\text { AASM } \\
2007\end{array}$ & $\begin{array}{l}57 \quad[31 \quad \text { Sleep } \\
\text { Apnea (SA), } 19 \\
\text { PLMS }]\end{array}$ \\
\hline $\begin{array}{l}\text { Cesari et al. } 2018 \\
{[27]}\end{array}$ & $\begin{array}{l}\text { Claimed to have avoided the } \\
\text { subjective nature of human- } \\
\text { defined LM specifications, } \\
\text { i.e., those set by AASM } \\
\text { standards for example, by } \\
\text { classifying between Periodic } \\
\text { Limb Movement Disorder } \\
\text { (PLMD) and control subjects } \\
\text { using non-parametric distri- } \\
\text { butions built off of features } \\
\text { extracted from Discrete } \\
\text { Wavelet Transform (DWT) } \\
\text { - decomposed EMG signals. } \\
\text { Given the unconventional } \\
\text { nature of their technique, } \\
\text { providing a one-to-one com- } \\
\text { parison with manually scored } \\
\text { signals would have offered a } \\
\text { clinically sound validation of } \\
\text { their technique [27]. }\end{array}$ & $\begin{array}{l}\text { "semi- } \\
\text { supervised } \\
\text { data-driven } \\
\text { approach" } \\
{[27]}\end{array}$ & $\begin{array}{l}63 \text { [36 PLMD, } 27 \\
\text { control] }\end{array}$ \\
\hline
\end{tabular}




\begin{tabular}{|c|c|c|c|}
\hline Authors & $\begin{array}{l}\text { Unique features and/or draw- } \\
\text { backs pertaining to the study }\end{array}$ & $\begin{array}{l}\text { PLM Stan- } \\
\text { dards }\end{array}$ & Dataset \\
\hline $\begin{array}{l}\text { Stefani et al. } \\
2017[28]\end{array}$ & $\begin{array}{l}\text { PLM count and scores were } \\
\text { distinguished between REM } \\
\text { and non-REM stages of sleep. } \\
\text { Validation was restricted to } \\
\text { individuals with AHI less } \\
\text { than } 5[28] \text {. }\end{array}$ & $\begin{array}{l}\text { AASM } \\
2012\end{array}$ & $\begin{array}{l}40 \text { [20 Restless } \\
\text { Leg Syndrome } \\
\text { (RLS), } 20 \text { con- } \\
\text { trol] }\end{array}$ \\
\hline $\begin{array}{l}\text { Huang et al. } 2015 \\
\text { [29] }\end{array}$ & $\begin{array}{l}\text { One-to-one comparison of } \\
\text { randomly selected PLMS } \\
\text { segments scored using their } \\
\text { automated software versus } \\
\text { visual scoring was provided. } \\
\text { Validation was only done } \\
\text { for individuals without sig- } \\
\text { nificant sleep-disordered } \\
\text { breathing events [29]. }\end{array}$ & $\begin{array}{l}\text { WASM/ } \\
\text { IRRLSG } \\
2006\end{array}$ & $\begin{array}{l}21 \quad[15 \text { RLS, } \\
\text { control] }\end{array}$ \\
\hline $\begin{array}{l}\text { Moore et al. } 2014 \\
{[30]}\end{array}$ & $\begin{array}{l}\text { Adaptive filters were used } \\
\text { to filter out noise interfer- } \\
\text { ences. Subjects with vari- } \\
\text { ous sleep disorders such as } \\
\text { REM Sleep Behavior Dis- } \\
\text { order (RBD), insomnia, etc. } \\
\text { were included. Respiratory } \\
\text { events were considered when } \\
\text { scoring for PLM, however, } \\
\text { the latter was accommodated } \\
\text { by modifying the standards } \\
\text { specified by domain experts. }\end{array}$ & $\begin{array}{l}\text { AASM } \\
2007\end{array}$ & $\begin{array}{l}60 \text { subjects from } \\
\text { Wisconsin Sleep } \\
\text { Cohort (WSC), } \\
18 \text { Subjects from } \\
\text { Stanford Sleep } \\
\text { Cohort (SSC) }\end{array}$ \\
\hline
\end{tabular}

another, but compelling PLM detection algorithm that offered clinicians with a simple, yet efficient tool to monitor PLM. In particular, the more recent specifications, i.e., that of the World Association of Sleep Medicine (WASM) 2016, stipulated by the joint task force of the International and the European Restless Legs Syndrome Study Groups (IRLSSG and EURLSSG), as reviewed in [7], was used. In fact, the authors in [7] provided more than just a summary of the clinical standards by clearly laying them down in a manner highly suited for software implementation [7].

Figure 3.1, depicted below, shows a snapshot of the application of the algorithm developed using [7] on a segment of raw EMG. Within the same figure, also shown is the result of applying 


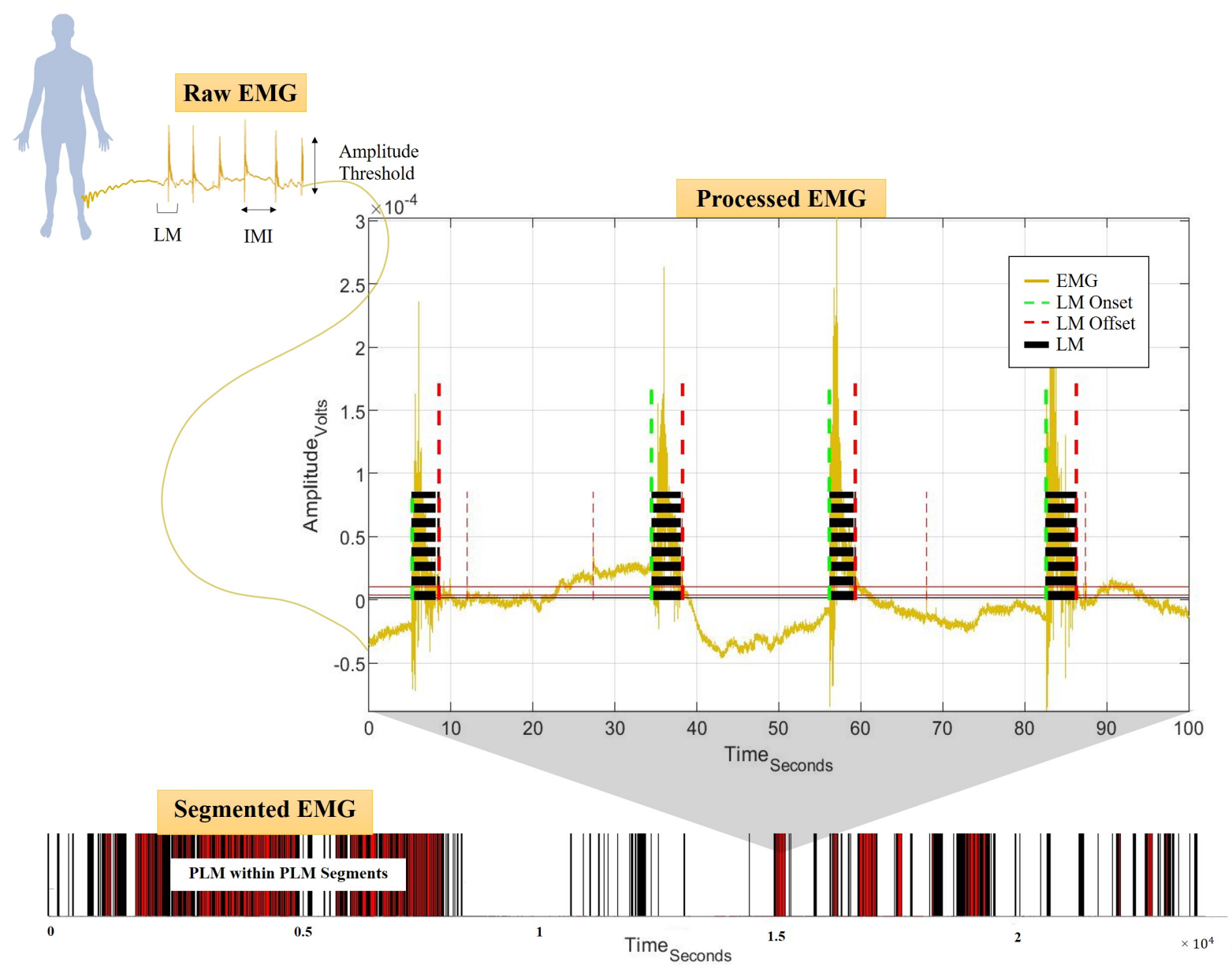

Figure 3.1: Developed PLM Detection Algorithm based on [7]

the algorithm on the entire EMG obtained from one of the limbs of a subject during one night of PSG. Note that, the latter displays the presence or absence of PLM with respect to time for the whole night of recording for the subject being analyzed. This is elaborated more in section 3.2.3. That being said, this Chapter is organized into 2 sections. Specifically, while the first section goes over a step-by-step implementation of [7], the second section demonstrates the results of applying the latter on actual datasets. 


\subsection{Method - Pre-processing, PLM Identification, Segmenta- tion and scoring}

\subsubsection{EMG pre-processing}

Before proceeding to detect PLM, it is significant to pre-process the signal under question. In line with the latter, since most of the PLM detection steps discussed later revolve around the concept of 'thresholds' [7], it is crucial that, as part of 'pre-processing', the given signal is first detrended, i.e., centered around zero along the y-axis. Specifically, illustrated in the 1st subplot of Figure 3.2, is a sample of detrended EMG signal. That being said, in order to remove the baseline wander from the signal being analyzed, a high pass filter set at $10 \mathrm{~Hz}$ can then be applied to the signal [7]. This is as shown in the 2 nd subplot of Figure 3.2. In conjunction with the latter, in order to discard noise from power-line interference, a notch filter can additionally be used. Though only slightly evident, this can be discerned from the 3rd subplot of Figure 3.2, in which, the thick band seen throughout the baseline of the sample signal in the 2nd subplot reduces to a certain extent in the 3rd subplot following the application of the notch filter. Lastly, as shown in the 4th subplot of Figure 3.2, the final 'pre-processing' step involves rectifying the signal being analyzed.

As a side note, it is of interest to mention here that, for all of the approaches discussed here, and hereafter, MATLAB [33] was the coding platform used. Also, unless specified otherwise, for all the signals being analyzed, the processes discussed were independently carried out on 5-minute portions of the signal. When necessary, the results from each of these segments were concatenated to correspond to the entire length of the signal being analyzed.

\subsubsection{LM (Leg Movement) Activity Identification}

Following pre-processing, the next step was to superimpose the thresholds defining the 'onset' and 'offset' of a LM onto the signal being analyzed. This is shown in Figure 3.3. In particular, as reviewed in [7], in order to qualify to be the start and end of a LM, the values in the signal under analysis had to be greater than or equal to $8 \mu \mathrm{V}$ above and less than $2 \mu \mathrm{V}$ above the resting 

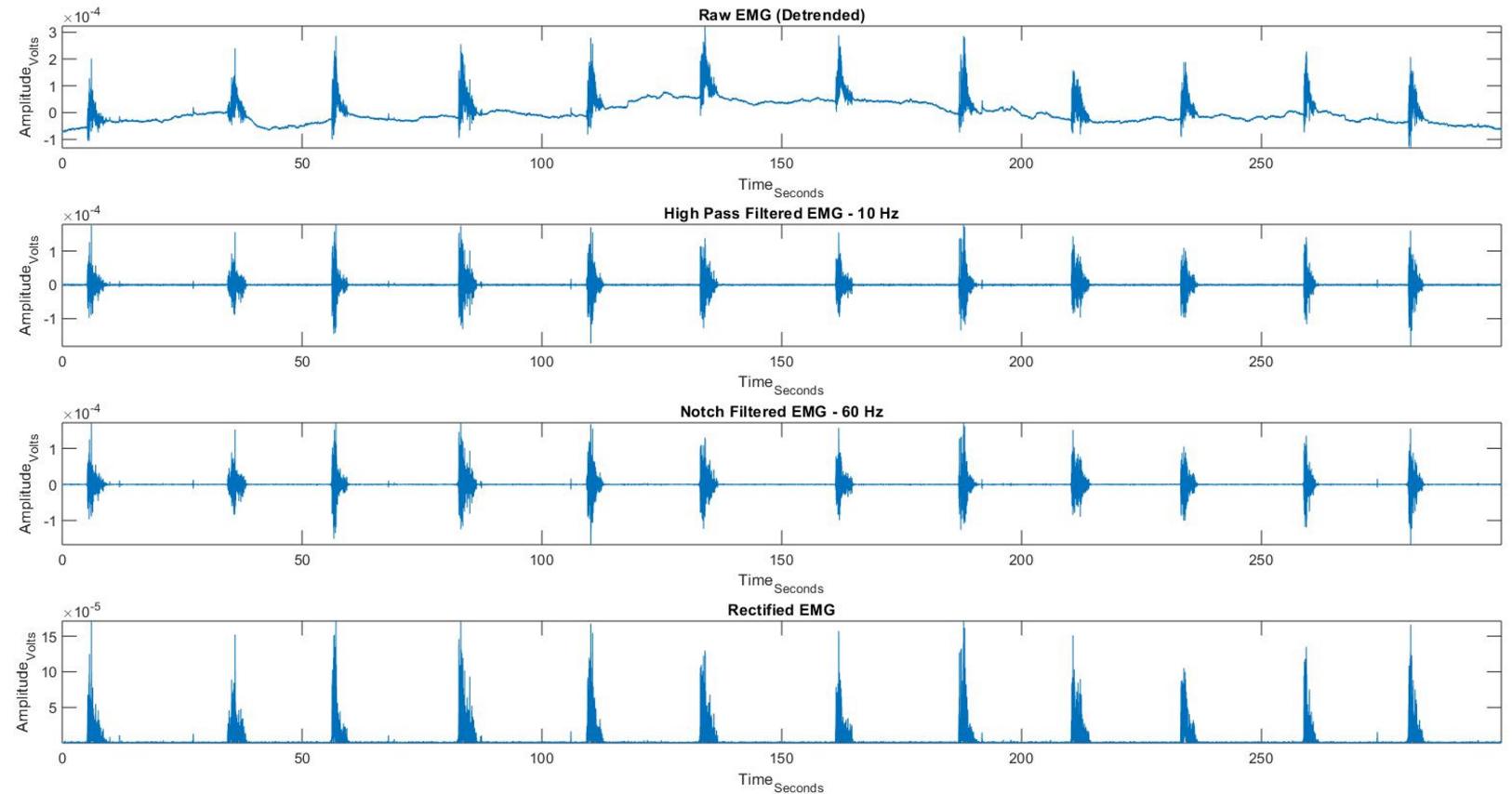

Figure 3.2: EMG pre-processing steps on a 5 min EMG segment from a subject

baseline, respectively. Additionally, in order to qualify to be the end of a LM, it was required that the amplitude following the end of the LM be less than $2 \mu \mathrm{V}$ above baseline, for a minimum of $0.5 \mathrm{~s}$. Notwithstanding, the identified LM also had to satisfy a morphology condition that required that the LM's median amplitude be greater than or equal to $2 \mu \mathrm{V}$ above resting baseline for at least $0.5 \mathrm{~s}[7]$. Aside, it is of interest to note here that, in order to apply these requirements onto the signal being analyzed, based on observations, the resting baseline was set to $2 \mu \mathrm{V}$. With that said, what follows is a detailed excerpt on the LM detection process built off of these specified conditions.

Specifically, to begin the process of LM detection on a $(5 \mathrm{~min})$ segment, all the values greater than the onset threshold of $8 \mu \mathrm{V}$ above baseline is marked. These are represented by the dashed green lines in part (i) of Figure 3.4. Note that, the latter is carried out to ensure that, out of all the possible onsets to choose from later on, only those that are above the onset threshold of $8 \mu \mathrm{V}$ above baseline, as required in [7], are left. Incidentally, these points are referred to by the name 'potential 


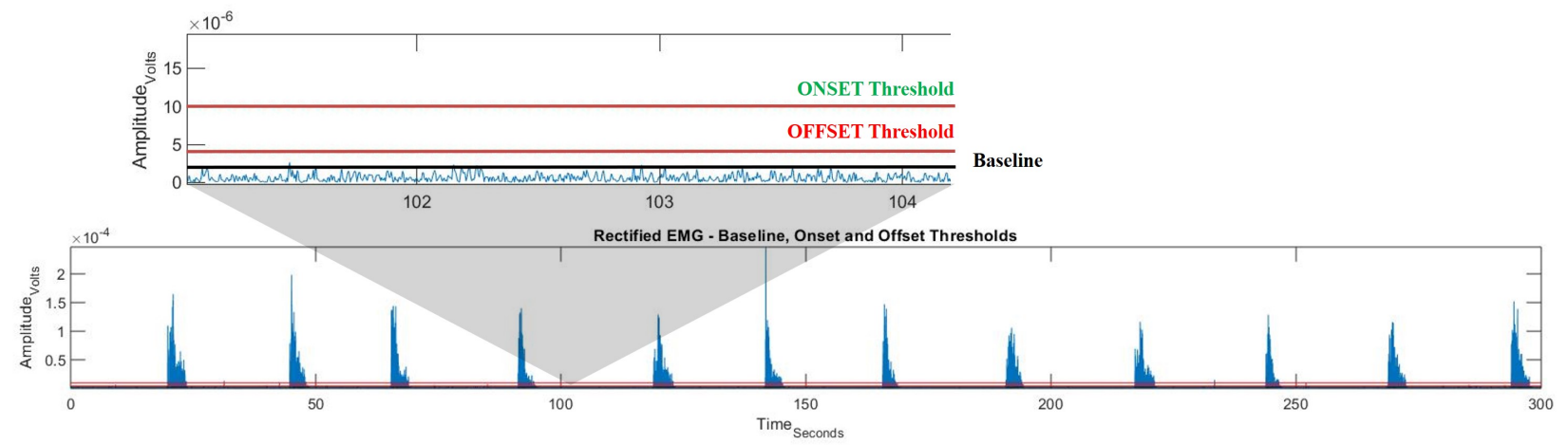

Figure 3.3: Baseline and LM Onset/Offset thresholds on a 5 min EMG segment from a subject

onsets' in the current discussion. Regardless, in order to facilitate the subsequent steps leading to the identification of possible offsets, the rectified EMG values greater than $8 \mu \mathrm{V}$ above baseline that are separated by $0.5 \mathrm{~s}$ or more are temporarily isolated. This is as shown in part (ii) of the figure where only the values from part (i) that were $0.5 \mathrm{~s}$ apart or more are shown. For discussion sake, these retained values are referred to as $\mathrm{ON}$ boundaries.

Moving on to the next step, within the region enclosed by any $2 \mathrm{ON}$ boundaries per segment, all the values that are less than the offset threshold of $2 \mu \mathrm{V}$ above baseline is marked. Explicitly, the latter is depicted by dashed red lines in part (iii) of Figure 3.4. Moreover, of the points marked as less than the offset threshold of $2 \mu \mathrm{V}$ above baseline from part (iii), only the first value within each bounded region that lasted for at least $0.5 \mathrm{~s}$ is kept for further processing. This is to ensure that the only available offsets to choose from later on are those that are less than $2 \mu \mathrm{V}$ above baseline lasting for less than $0.5 \mathrm{~s}$, as stated earlier [7]. Nevertheless, such selected points in the current thesis are referred to as OFF boundaries and are as shown in part (iv) of the figure by dashed red lines. Note that, in order to accommodate instrumentation noise for example, the requirement of having to be less than $2 \mu \mathrm{V}$ above baseline for $0.5 \mathrm{~s}$ was relaxed such that, for a given value that ended up being less than $2 \mu \mathrm{V}$ above baseline, it was only required that the latter was less than the $2 \mu \mathrm{V}$ above baseline mark for $90 \%$ of the 0.5 s duration limit.

Nonetheless, within the region enclosed by any 2 OFF boundaries per segment, a possible LM was identified, if and only if, from the set of potential onset values from before, there was at least 

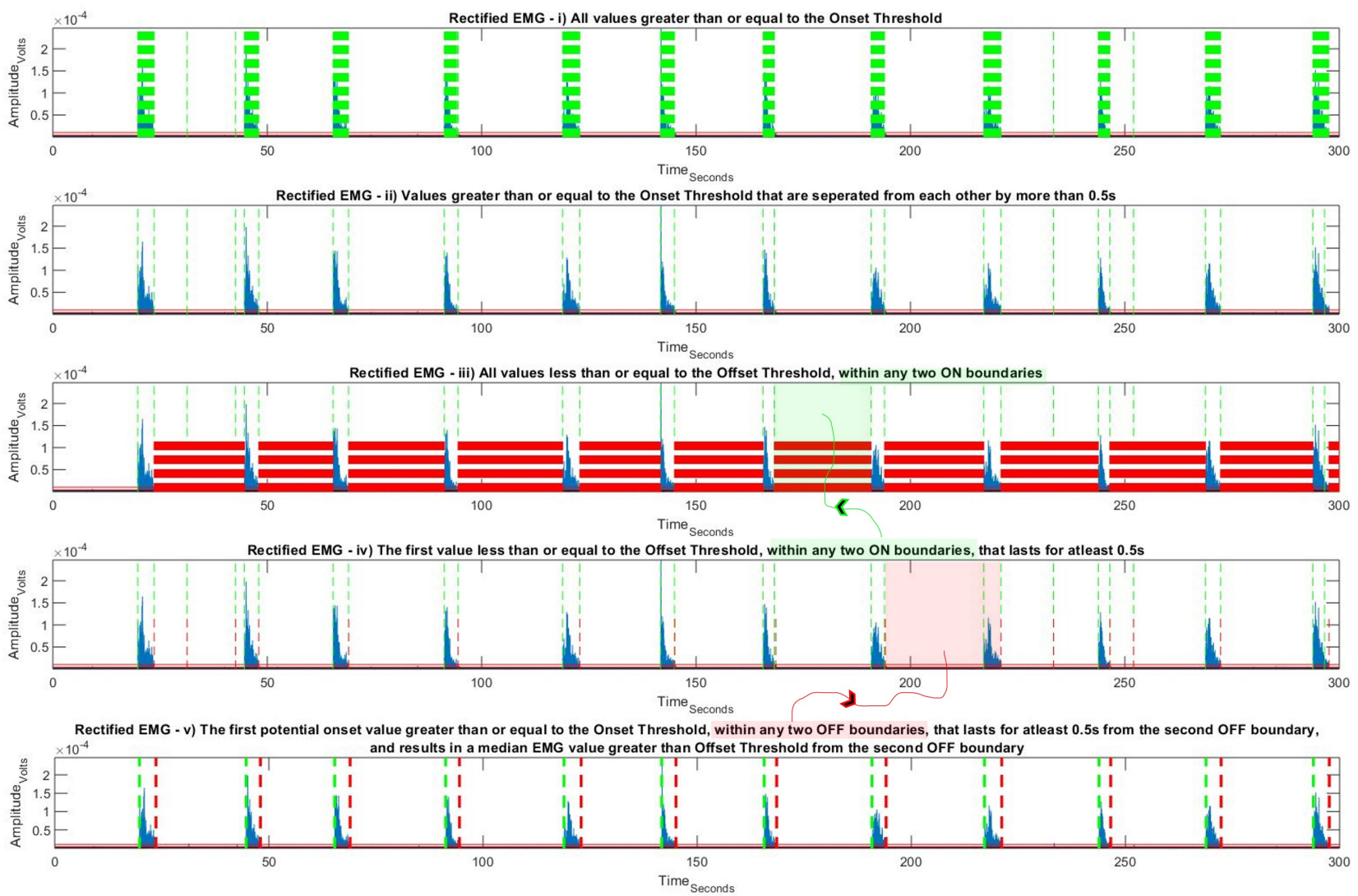

Figure 3.4: Implementation of Ferri et al.'s [8] PLM detection process on a 5 min EMG segment from a subject

one value that was $0.5 \mathrm{~s}$ apart from the second OFF boundary, and the former resulted in a median EMG value greater than the offset threshold of $2 \mu \mathrm{V}$ above baseline between itself and the second OFF boundary. This is implemented so as to only capture LM that satisfied the minimum LM duration requirement as well as those that satisfied the minimum morphology requirement, as stated in [7]. Note that, for all the potential onsets that existed between any two OFF boundaries, only the first potential onset that satisfied the minimum $0.5 \mathrm{~s}$ duration limit and morphology requirement is chosen and the rest are discarded from further analysis. In the event that none of the requirements stated were satisfied by any of the potential onsets, the possibility of a LM between that respective onset and OFF boundary under question was dropped. Part (v) of Figure 3.4 shows in bolded green and red respectively, the chosen onsets and offsets of identified LM for the segment under 
analysis. Figure 3.5 is the same plot as part (v) of Figure 3.4 except that, instead of the rectified EMG, raw EMG is shown beneath the captured LM to highlight the progress from raw EMG to algorithm-detected LM.

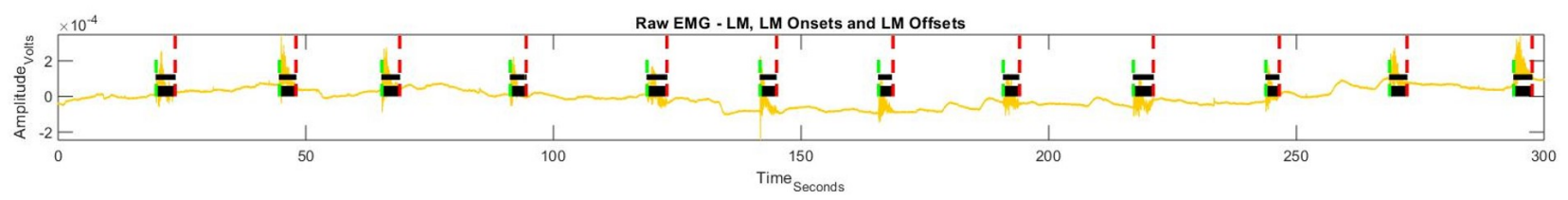

Figure 3.5: PLM identified on a 5 min EMG segment from a subject

With all that said, the overall process for LM identification stated above is repeated for all the 5-minute EMG segments withdrawn from a subject. The resulting LM information extracted per segment are chained together to produce a complete set of LM gathered per subject with respect to time, for the entire duration of the time spent in bed by that subject. As a side note, the first value and the last value per segment is artificially induced to be an OFF and ON boundary respectively so as not to miss out on the LM that happen at the beginnings and ends of segments, when carrying out the analysis described above per segment.

\subsubsection{Mono-lateral PLM Identification and Segmentation}

Moving on to identifying whether a detected LM in a given segment qualified to be part of a PLM series, the latter needs to be checked against 3 requirements: (1), that the LM under consideration doesn't last longer than 10s, (2), the Inter-Movement Interval (IMI), i.e., the duration between any 2 of the LM satisfying condition (1) is 10 to 90 s and (3), that there is a minimum of 4 such LM, all satisfying conditions (1) and (2), occurring consecutively [7]. Note that, as specified in [7], LM that satisfy (1) are referred to as Candidate Limb Movement (CLM), i.e., more precisely, monolateral Candidate Limb Movement (mCLM) since, only LM from either the left or right leg are being considered at any one given time. If all 3 conditions are met, the latter is then formally referred to as PLM [7]. 
The above is visually illustrated in Figure 3.6, in which, one and less than half a portion of a PLM segment taken from a subject is shown. As quantified in the tables, the first three columns represent the start times, stop times and duration of LM captured for the chosen PLM segments. Moreover, the IMI between any 2 LM is shown in the IMI column which, to be exact, for any given row of LM, shows the IMI between itself and the LM right before it. That aside, separate columns are designated to tabulate the fulfilment of the first 2 conditions. Lastly, the fulfillment of condition 3 is captured by the PLM count column which, is coded for such that, the count of PLM begins, if and only if, there were at least 4 consecutive LM that satisfied the LM and IMI time limits stated by conditions (1) and (2). To be precise though, this is except for the first LM of the batch that only requires condition (1) to be satisfied since, being the first LM of the series, it wouldn't have a LM before it to have an IMI with.

That said, this brings us to the discussion on what constitutes the first LM of a PLM series, or in other words, signifies the start of a PLM segment. As implied in [7], one possibility by which a given LM can end up being the first LM in a PLM series is if the LM in question happens to be the first CLM as well as the very first LM from the entire sleep duration following which there are successive CLM that satisfied conditions (1) and (2). Another possibility is if the LM in question fails to satisfy condition (2), i.e., the IMI duration requirement, between itself and the LM right before it. The latter holds true provided that the LM right before the LM in question had qualified to be a CLM, or in other words, had satisfied condition (1) [7]. This seems to be the case of the last LM from the first PLM segment and the first LM from the second PLM segment shown in Figure 3.6. To elaborate, although the last LM from the first PLM segment passed the LM duration limit dictated by condition (1), the IMI duration between itself and the LM following it fails to meet the IMI duration limit dictated by condition (2), hence the need to start a new segment, i.e., the second PLM segment, in this case. A final possibility by which a given LM could end up being the first LM in a PLM series is, if the LM before the LM in question did not satisfy the LM duration limit dictated by condition (1) [7]. The latter would hold true regardless of whether the IMI duration limit, i.e., condition (2) was satisfied or not since, if condition (1) was not met, it wouldn't make sense to start a PLM segment with a non-CLM regardless. 
0 or 1 where

1 indicates that IMI duration is $\geq 10$ s and $\leq 90 \mathrm{~s}[7]$

0 or 1 where

1 indicates that $\mathrm{LM}$ duration is $>0.5 \mathrm{~s}$ and $\leq 10 \mathrm{~s}$, i.e., otherwise known as, or qualifies to be a CLM [7]

\section{PLM Segment}

\begin{tabular}{|c|c|c|c|c|c|c|c|}
\hline $\begin{array}{c}\text { LM } \\
\text { Start Time }\end{array}$ & $\begin{array}{c}\text { LM } \\
\text { Stop Time }\end{array}$ & $\begin{array}{c}\text { LM } \\
\text { Duration }\end{array}$ & $\begin{array}{c}\text { Requirement } \\
1\end{array}$ & IMI & $\begin{array}{c}\text { Requirement } \\
2\end{array}$ & $\begin{array}{l}\text { PLM } \\
\text { Count }\end{array}$ & \multirow{13}{*}{$\begin{array}{l}\text { Requirement } \\
3 \\
\geq 4 \text { consecutive } \\
\text { CLM [7] }\end{array}$} \\
\hline 1291.7 & 1293.9 & 2.1914 & 1 & 728.5 & 0 & 1 & \\
\hline 1320.9 & 1323.5 & 2.5977 & 1 & 29.215 & 1 & 2 & \\
\hline 1351.4 & 1354.1 & 2.6211 & 1 & 30.496 & 1 & 3 & \\
\hline 1380.1 & 1383.2 & 3.0781 & 1 & 28.688 & 1 & 4 & \\
\hline 1413.3 & 1416.7 & 3.375 & 1 & 33.164 & 1 & 5 & \\
\hline 1438 & 1440.9 & 2.9492 & 1 & 24.68 & 1 & 6 & \\
\hline 1463.3 & 1466.4 & 3.1133 & 1 & 25.375 & 1 & 7 & \\
\hline 1485.8 & 1488.8 & 2.9727 & 1 & 22.461 & 1 & 8 & \\
\hline 1513.5 & 1516.2 & 2.6641 & 1 & 27.742 & 1 & 9 & \\
\hline 1533.6 & 1535.3 & 1.6523 & 1 & 20.066 & 1 & 10 & \\
\hline 1556.1 & 1560.2 & 4.0508 & 1 & 22.527 & 1 & 11 & \\
\hline 1579.9 & 1583 & 3.1406 & 1 & 23.754 & 1 & 12 & \\
\hline
\end{tabular}

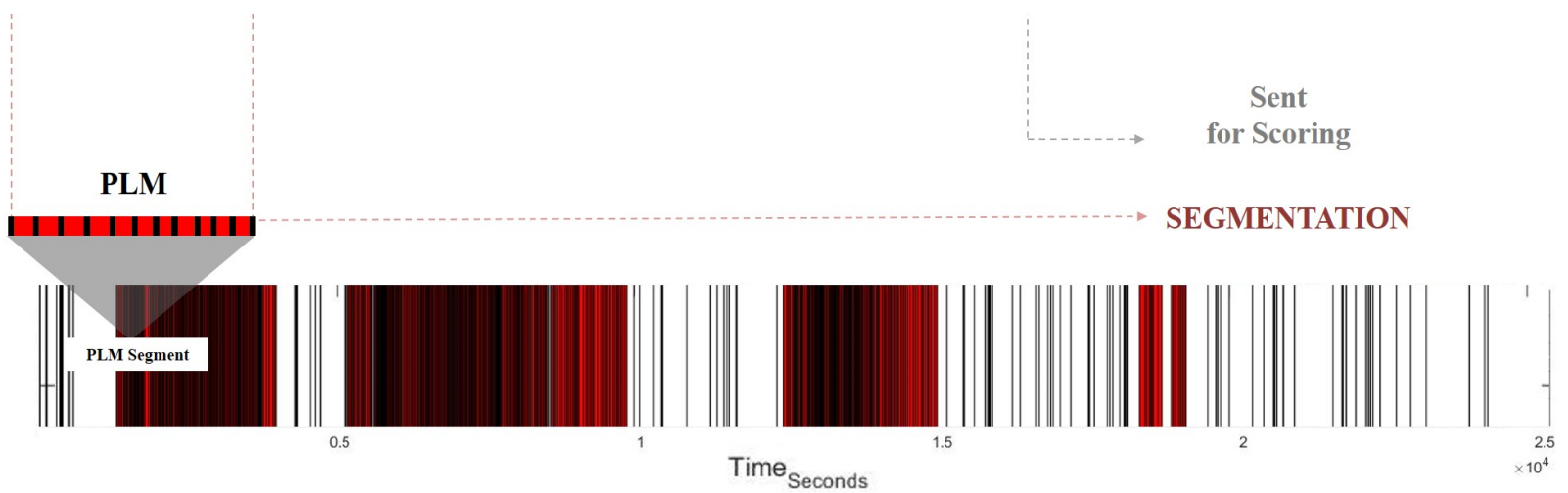

Equivalent time scale - Hours

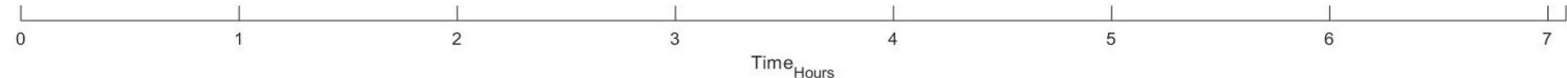

Indicates start of a new PLM segment since,

IMI requirement 2 not satisfied. Hence, LM does not qualify to be part of the previous PLM segment, in this case.

Next PLM Segment

1602.5

1.5117

3.5156

$1630.3 \quad 4.1836$

1626.1

3.957

1649.3

1653.3

2.5938

\begin{tabular}{|l|l|}
\multicolumn{1}{c|}{} & \\
\hline 4.6914 & 0 \\
\hline 17.938 & 1 \\
23.613 & 1 \\
23.207 & 1 \\
22.043 & 1 \\
\hline
\end{tabular}

1
2
3
4
5

Figure 3.6: Example of one and less than half a portion of a PLM segment taken from a subject Implementation of PLM specifications summarized by [7] for mono-lateral limbs 
Just like the first LM of a PLM segment, a given LM can end up being the last LM of a PLM segment if the LM right after the LM in question ceases to satisfy either the LM duration limit dictated by condition (1), or the IMI duration limit specified by condition (2) [7]. Moreover, if there were no LM following the LM in question, i.e., in other words, if the LM in question was the last CLM as well as the final LM from the entire sleep duration that was preceded by a series of CLM that had satisfied conditions (1) and (2), then the given LM can be that of the last LM of a PLM segment [7].

Now that it is known how to extract PLM segments and the corresponding LM that make up the latter, it would be of interest to visualize the portions of time occupied by the LM from each limb for the entire duration of time spent in bed for each subject. For instance, the 'LM-time' map depicted in the middle of Figure 3.6 shows how the 12 LM from the first of the 2 PLM segments discussed earlier fit in with respect to the portions of time occupied by all the other LM from the left limb of the subject. Specifically, while streaks of black lines in the plot only represent portions of time occupied by individual LM, black lines with red background represent the time occupied by LM that had qualified to be PLM. Aside from that, it is also of interest to mention here that the count of PLM within the time boundaries of every PLM segment that exist per subject is further used for PLM scoring purposes. This is discussed much later in section 3.3.

\subsubsection{Bi-lateral PLM Identification and Segmentation}

To start with, at any one given instant, (i), only a maximum of 4 mCLM from opposite legs can be combined into 1 [7]. An example of, in this case, 2 mCLM from opposite legs that are combined into 1 is shown in Figure 3.7. As illustrated in the figure, not only is there a limit on the number of mCLM that can be combined into 1, there is also a time constraint as to how they can occur with respect to each other. The latter can be checked for by first identifying the LM that occurs after the start of the LM from the opposite limb. More importantly, if that chosen LM also satisfies the condition of starting within $0.5 \mathrm{~s}$ from the end of the LM from the opposite limb, the $2 \mathrm{LM}$ qualify to be merged into 1 [7]. For example, in Figure 3.7, the LM from the left limb is identified to be the limb that occurs after the onset of the LM from the right limb. Since the left limb 
also satisfies the condition of starting within $0.5 \mathrm{~s}$ from the end of the LM from the other (right) limb, the 2 LM become eligible to be considered for being combined into 1. Following condition (i), condition (ii) requires that none of the LM to be combined are to be greater than 10s which, inherently, is the defining feature of mCLM [7]. Going back to Figure 3.7, neither of the CLM being combined are beyond the 10s upper limit. Lastly, as depicted towards the end of Figure 3.7, condition (iii) requires that the total duration of the combined LM, that can now be referred to as bi-lateral Candidate Limb Movement (bCLM), does not last more than 15s [7]. This, in fact, is the case for the 2 LM being combined into 1 bCLM in the figure.

With all that said, depending on whether all three conditions (i), (ii), and (iii) are met, CLM may become bCLM or remain as MCLM after superimposing the LM from both the legs. Nevertheless, following the latter, the same conditions (2) and (3), as stated previously for mono-lateral PLM identification, is checked for to complete the bi-lateral PLM identification process [7]. In other words, regardless of whether the CLM became bCLM or remained as MCLM following superimposition, they are checked to see whether they satisfy the IMI duration limit of 10 to 90 s duration, as well as, whether they co-occur with a minimum of 3 other CLM respectively, before they are designated as PLM in the bi-lateral context.

Figure 3.8 shows the portions of time occupied by all the LM from both the limbs of a subject for the entire duration of time spent in bed by that subject. As stated earlier, while the black lines in the plot represent portions of time occupied by individual LM, the black lines with red background represent the same for LM that had qualified to be PLM. In addition to the LM from the individual limbs, a 'LM-time' plot is also depicted for the LM formed following superimposition and merging of LM from both the limbs, where applicable.

That aside, note that the time spent in bed may not necessarily mean time spent sleeping which, is crucial to distinguish when scoring for PLMS. Specifically, when scoring for PLMS, only PLM that occur during the time spent sleeping is considered [7]. In fact, depending on whether they were needed by whomever analyzing, those that occur during 'awake time' are designated a separate score, i.e., Periodic Limb Movement during Wake (PLMW) [7]. That said, this brings us to the next section on scoring for PLM based on the PLM detected thus far per subject. 
The onset of the LM from one of the limbs to be combined should occur after the onset of the LM of the other limb, as well as, the former should occur before $0.5 \mathrm{~s}$ from the end of the LM from the other limb, respectively [7].

i) Combination of $\leq 4$ mono-lateral CLM [7]

2 CLMs are shown in this case
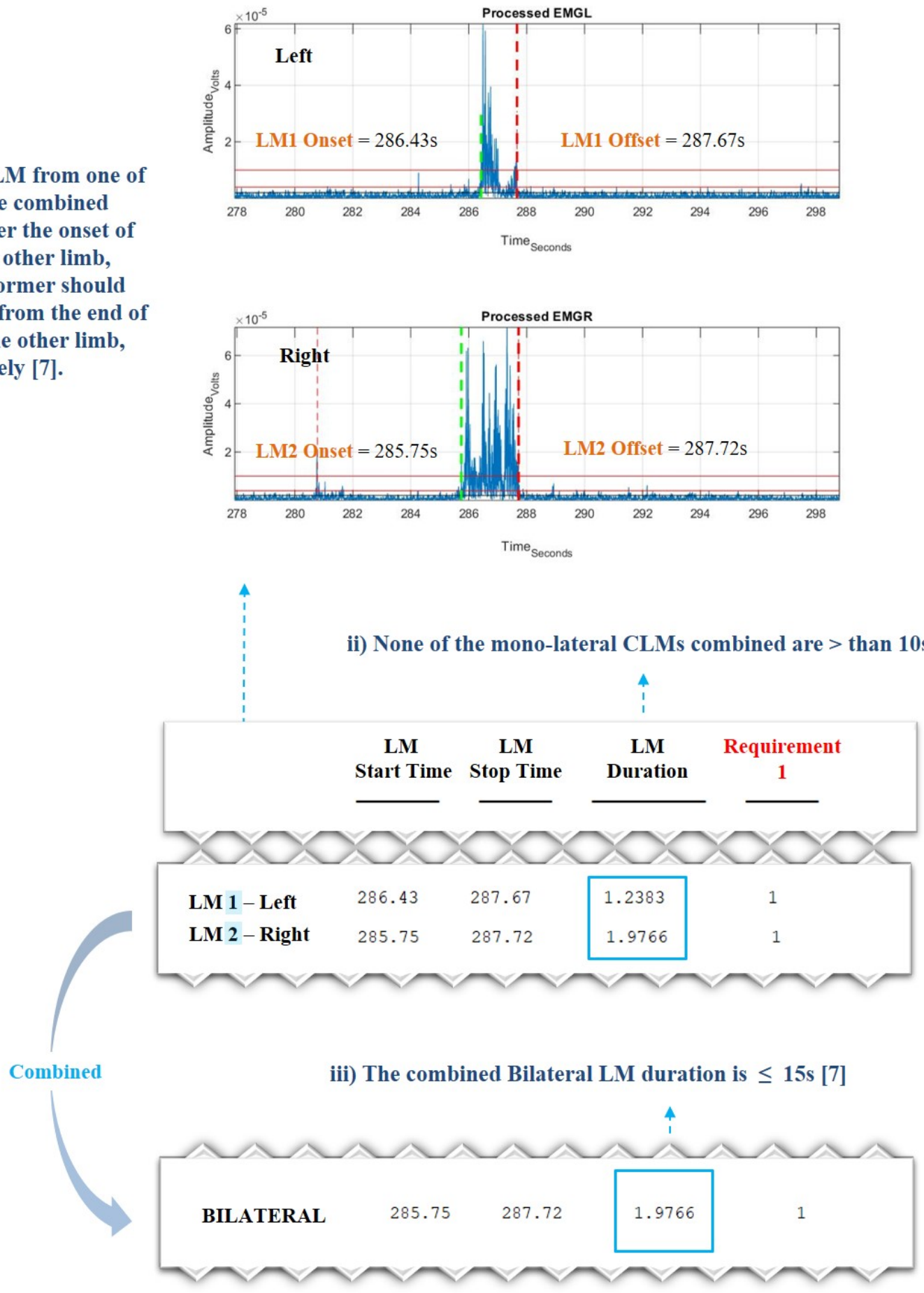

Figure 3.7: Example of 2 LM from opposite limbs from a subject - Implementation of PLM specification summarized by [7] for bi-lateral limbs 


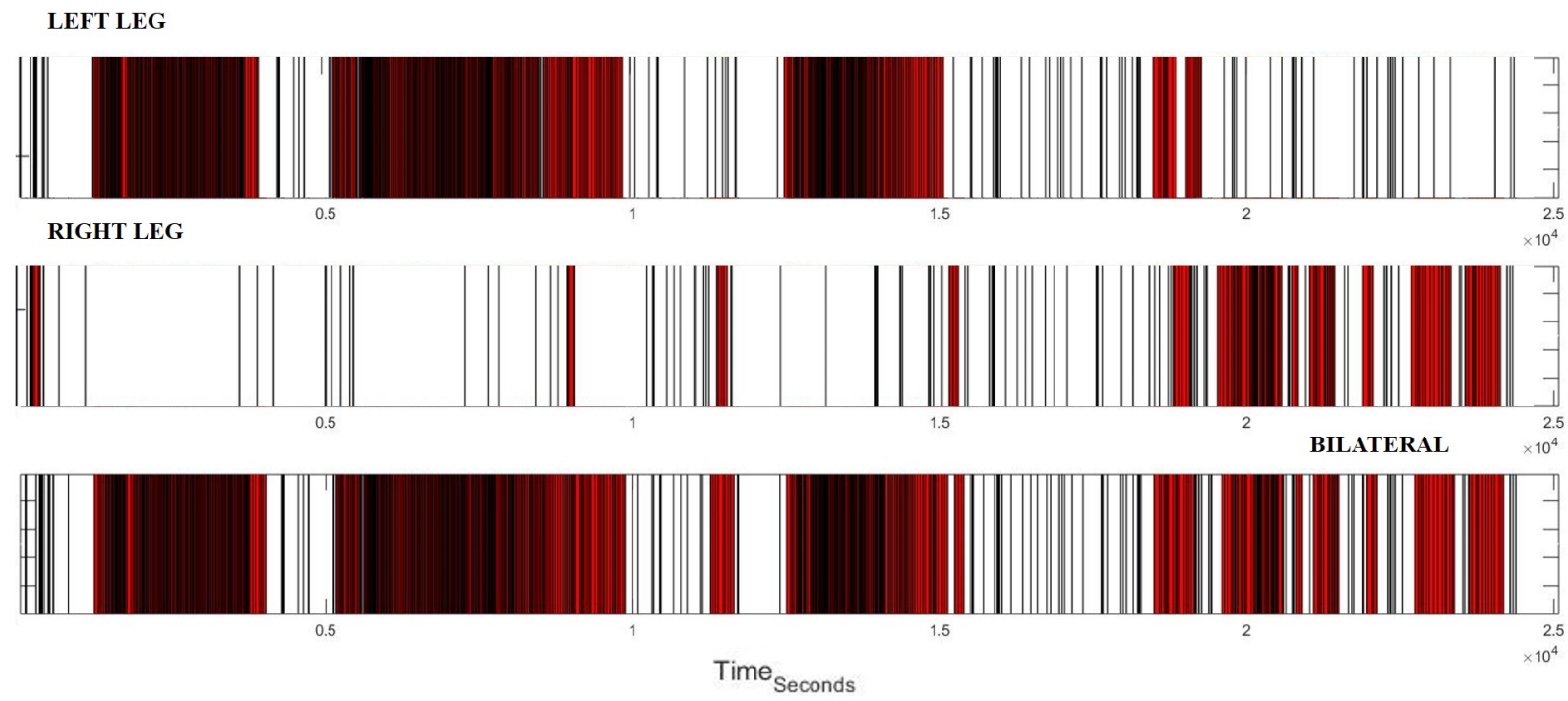

Equivalent time scale - Hours

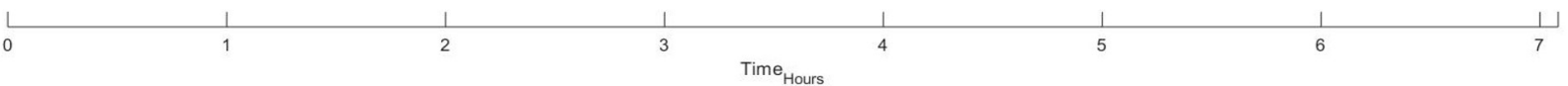

Figure 3.8: Occurrence of PLM segments identified mono-laterally and bi-laterally for a subject during one PSG session.

\subsection{Results and Discussion - Analysis}

\subsubsection{Comparison of Clinical vs. Algorithm Scores}

As briefly mentioned above, the calculation of PLMS per subject is given by the following formula [7]:

$$
\text { PLMS }=\frac{\text { Number of PLM that occurred during Sleep }}{\text { Hours of Sleep }}
$$


Using 3.1, separate scores are calculated for each leg, or bilaterally, based on the total number of PLM gathered per subject from sections 3.2.3 and 3.2.4 respectively. As indicated in 3.1, and mentioned in the previous section, only the PLM that corresponded to the time spent sleeping per subject is used to calculate PLMS [7]. To accomplish the latter, the hypnogram data attained per subject during PSG, indicating periods of wake versus sleep time, is used. Moreover, in the occasional instances where the captured LM from a PLM series corresponded to both awake and sleep times, the sleep stage that half, or more than half the portion of the LM in question corresponded to was chosen to be the assigned state of sleep in which the given LM was in. For example, if 80 percent of the LM in question from a given PLM series from a subject fell under sleep time, the former was designated to be occurring during sleep, and thus, was counted towards the scoring of PLMS for that subject. That aside, the total sleep time per subject was determined by accumulating all the periods of time that corresponded to sleep in the hypnogram data.

Nonetheless, before moving on to the results of applying the above to the datasets $\mathrm{A}$ and $\mathrm{B}$ (contributed courtesy of Sunnybrook hospital), whose demographics is summarized in Tables 3.2 and 3.3, it is of importance to state here that aside from discarding the PLM that corresponded to awake time, the clinical-based PLM scoring also discarded the PLM that corresponded to respiratory events. The specifics for discarding such PLM was based on the criteria dictated by the American Academy of Sleep Medicine (AASM) Manual for the Scoring of Sleep and Associated Events: Rules, Terminology and Technical Specifications, 2007 [34]. That being said, on a different note, it is of interest to mention here that, as the data from the two Datasets A and B were collected at two different time periods, the results for the two are presented separately.

With that said, since it is beyond the scope of the present thesis to detect respiratory events, one of the limitations of the currently developed PLM detection algorithm is that it does not discard PLM corresponding to respiratory events. Hence, the latter implies that, a comparison of the algorithm-based scoring with the clinical-based scoring, especially for those subjects who had a high number of respiratory events (reflected by their high Apnea-Hypopnea (AHI) scores), might not be too informative on the performance of the algorithm per se since one includes, while the other discards the PLM associated with respiratory events. Nonetheless, in the results and discus- 
Table 3.2: Demographics of Dataset A:

$$
\begin{gathered}
\mathrm{N}=56 \\
(\mathrm{M}=32 \text { and } \mathrm{F}=24)
\end{gathered}
$$

\begin{tabular}{|c|c|c|}
\hline & Median & Range \\
\hline Age & 56 & 19 to 77 \\
\hline PLM Index Bilateral & 4.1 & 0 to 75.4 \\
\hline PLM Index ${ }_{\text {Left }}$ & 1.6 & 0 to 75.2 \\
\hline PLM Index Right & 0.8 & 0 to 68.4 \\
\hline AHI Index & 5.1 & 0 to 93.9 \\
\hline SE $(\%)$ & 71.4 & 11.8 to 96.5 \\
\hline BMI & 28.4 & 18.5 to 45.5 \\
\hline
\end{tabular}

Table 3.3: Demographics of Dataset B:

\begin{tabular}{|c|c|c|}
\hline & Median & Range \\
\hline Age & 47.6 & 16.9 to 83.9 \\
\hline PLM Index Bilateral & 1.5 & 0 to 176.5 \\
\hline PLM Index ${ }_{\text {Left }}$ & 0 & 0 to 149.9 \\
\hline PLM Index ${ }_{\text {Right }}$ & 0.9 & 0 to 122.1 \\
\hline AHI Index & 1.1 & 0 to 64.9 \\
\hline SE $(\%)$ & 79.1 & 29.2 to 95.3 \\
\hline BMI & 26.1 & 19 to 46.4 \\
\hline
\end{tabular}

$$
\begin{gathered}
\mathrm{N}=38 \\
(\mathrm{M}=20, \mathrm{~F}=17, \text { Undefined }=1)
\end{gathered}
$$

sion sections that follow, brief speculations on the relationship between the algorithm vs. clinical PLM scores for those individuals with AHI greater than or equal to 5 is still provided.

Results and Discussion I (for the individuals with AHI $\geq 5$ ) 
Note that, to contemplate on the spread of PLM scores for the individuals with AHI greater than or equal to 5, as represented by the scatter of points in Figures 3.9 and 3.10, some extreme case scenarios are adopted below.

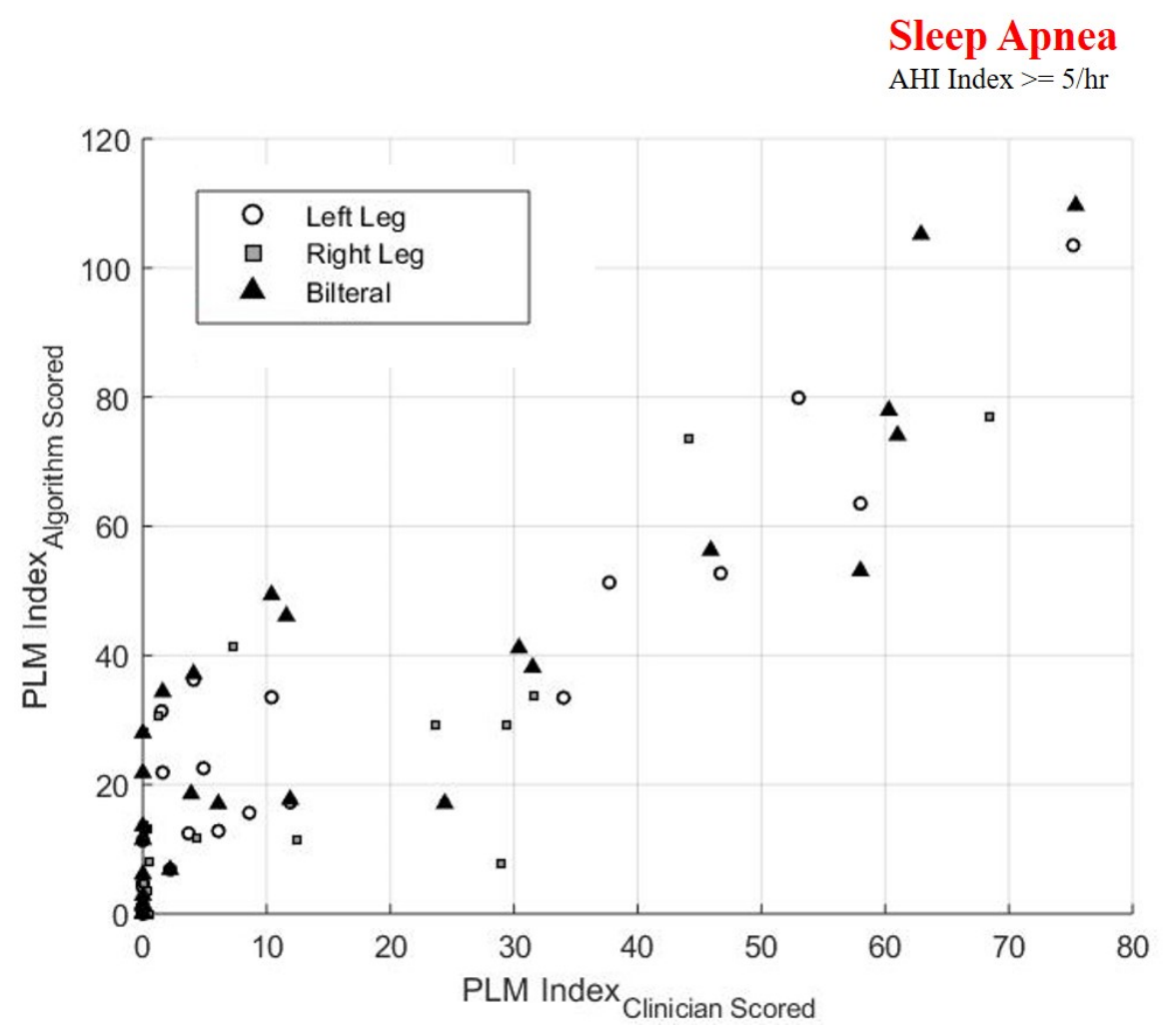

Figure 3.9: Dataset A - Clinical vs. Algorithm scores for 28 (out of 56) subjects (AHI $\geq 5$ )

To start with, in the instance that all the PLM that occurred in a subject occurred parallel to respiratory events, it is expected that the clinically-assigned PLM Index would be close to nil while the algorithm-scored PLM would be as high as the amount of PLM present in that subject. This is due to the inclusion of the respiratory-associated PLM in the latter which is otherwise completely discarded in the clinical scoring. As seen in the Figures 3.9 and 3.10, these are represented possibly by the spread of data close to the 'nil' scored clinical indices for the individuals with AHI greater than or equal to 5 from datasets $\mathrm{A}$ and $\mathrm{B}$, respectively.

Moreover, in the instance that none of the PLM occurred parallel to respiratory events, it is expected that the algorithm-scored PLM Index and its clinical counterpart would closely match 


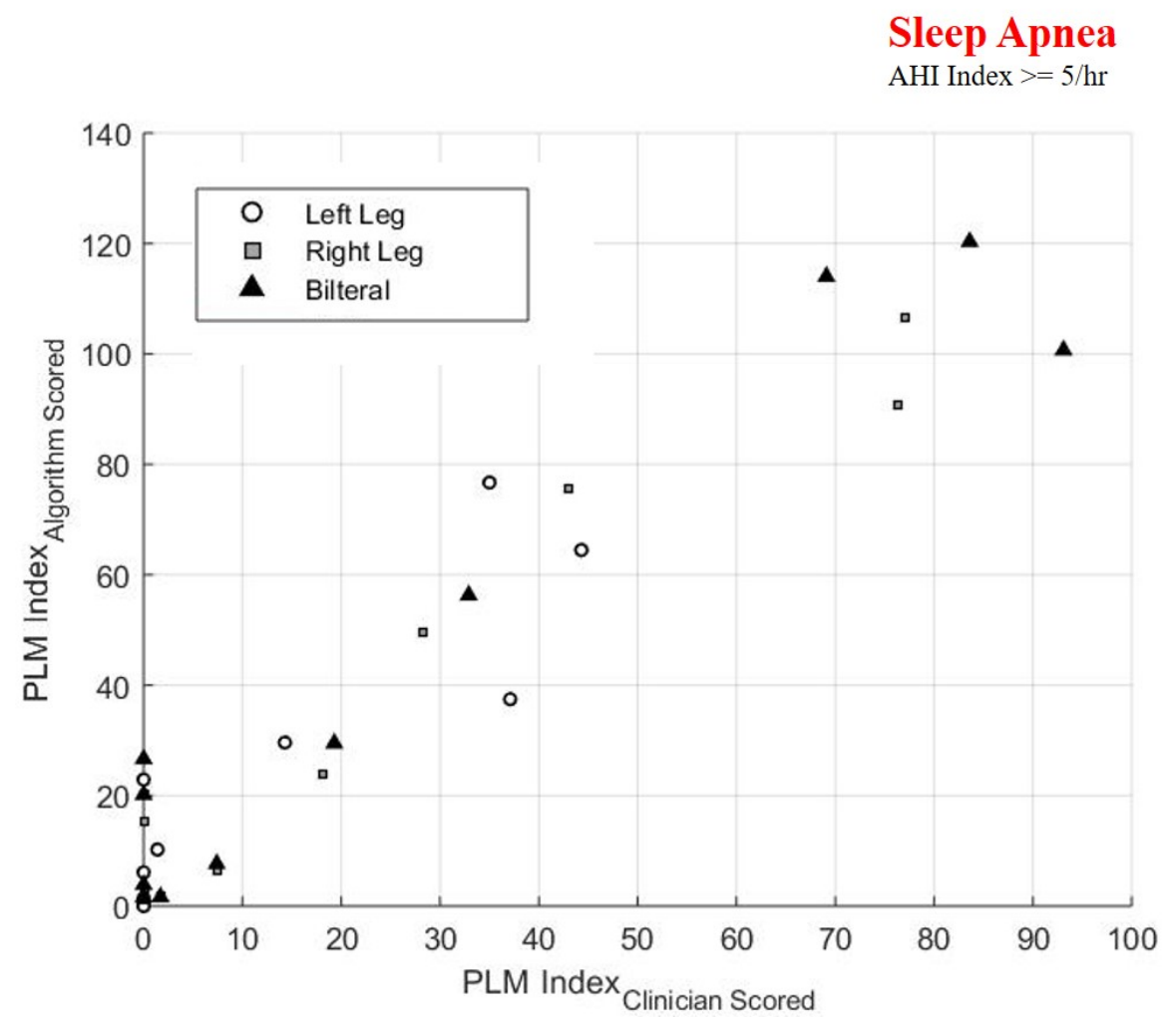

Figure 3.10: Dataset B - Clinical vs. Algorithm scores for 11 (out of 38) subjects (AHI $\geq 5$ )

each other. The idea here is that since the PLM occurs independent of respiratory events, that there would no PLM associated with respiratory events discarded from the clinical scoring. Hence, this would result in clinical scores not too different from the PLM scores calculated using the algorithm. Nonetheless, this trend can only be seen for a handful of the subjects from datasets A and B with AHI greater than or equal to 5, as seen in the Figures 3.9 and 3.10, thereby implying a lower likelihood that the 2 events occur independent of each other in individuals diagnosed with both sleep apnea and PLMS.

With that said, it is of interest to speculate on how the algorithm scores might differ from their clinical equivalent if, instead of all or none, some of the PLM co-occurred with respiratory events. In the latter case, it is highly likely that the algorithm-scored PLM Index would result in a higher score than its clinical counterpart. Specifically, the algorithm-scored PLM Index might be slightly higher or much higher than its clinical equivalent depending on the number of PLM co-occurring 
with the respiratory events. Incidentally, this trend can be construed from the generally higher algorithm versus clinical scores depicted in Figures 3.9 and 3.10. In essence, the latter implies a higher likelihood that the 2 events co-occur, at least for some of the PLM taking place per subject, in the individuals diagnosed with both sleep apnea and PLMS.

Nevertheless, it is expected that for future work, for the individuals with AHI Index greater than or equal to 5, the PLM corresponding to 'Respiratory events' would be found, and that the algorithm-scored PLM Index score would be modified to discard the PLM corresponding to 'respiratory events' in the manner specified by AASM, 2007 [34] for example, or going forward, using the latest available manual in the series. By doing so, it is expected that the latter would allow us to affirm whether the interpretations made thus far were correct. More importantly, the latter would allow us to benchmark the currently developed algorithm with the technician-based scoring without discarding the individuals with high sleep apnea index.

\section{Results and Discussion II (for the individuals with AHI $<5$ )}

Since for the individuals with sleep apnea index less than 5, the effect of the respiratory events on PLM is expected to be minimal, it is expected that the performance of the algorithm can be benchmarked against the clinical score by using the scores from these individuals. With that said, a breakdown of the parameters computed for the scores from the individuals with AHI less than 5 were as follows.

\section{Spearman Correlation}

To start with, for the subset of individuals from datasets A and B with AHI less than 5, the Spearman correlation coefficient, which evaluates the strength and direction of the relationship between 2 variables [35], is calculated using:

$$
r_{s}=\frac{\sum_{i=1}^{n}\left(x_{i}-\bar{x}\right)\left(y_{i}-\bar{y}\right)}{\sqrt{\sum_{i=1}^{n}\left(x_{i}-\bar{x}\right)^{2} \sum_{i=1}^{n}\left(y_{i}-\bar{y}\right)^{2}}}
$$


In equation 3.2, while $x_{i}$ or $y_{i}$ represent the individual ranks of the values from each set of scores, $\bar{x}$ or $\bar{y}$ depict the overall mean of the ranks from the respective set of scores. In the event that there were tied ranks, i.e., equal ranks, within either sets of scores, the latter is replaced with the mean of the respective set of tied ranks [35].

Analogous to the above, the scatter plot of the scores for these individuals, along with their linear regressions (that will be discussed in the next sub-section), are as shown in Figures 3.11, 3.12, and 3.13. Note that, like Figure 3.12, Figure 3.13 also represents the scatter plot and linear regression of scores from dataset $\mathrm{B}$, but the latter discards the scores from 2 subjects deemed as outliers, i.e., whose algorithm scores were almost twice as high as, or half as high as, their clinical equivalent.

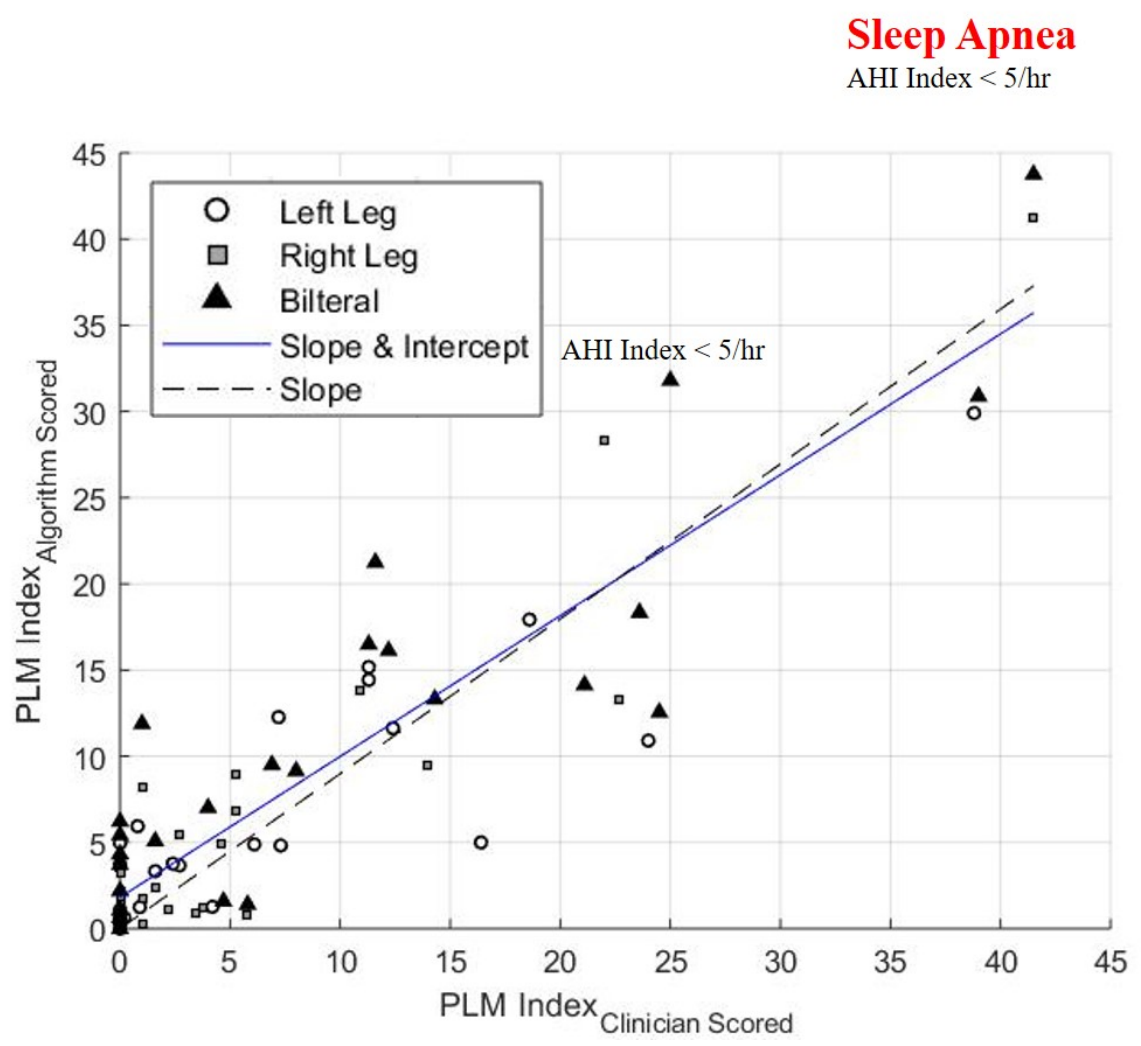

Figure 3.11: Dataset A - Clinical vs. Algorithm scores for 28 (out of 56) subjects $(\mathrm{AHI}<5$ )

With that said, it is of interest to state here that Spearman correlation was chosen over the more commonly used Pearson correlation since the scores from this study did not follow a bivariate 
Sleep Apnea

AHI Index $<5 / \mathrm{hr}$

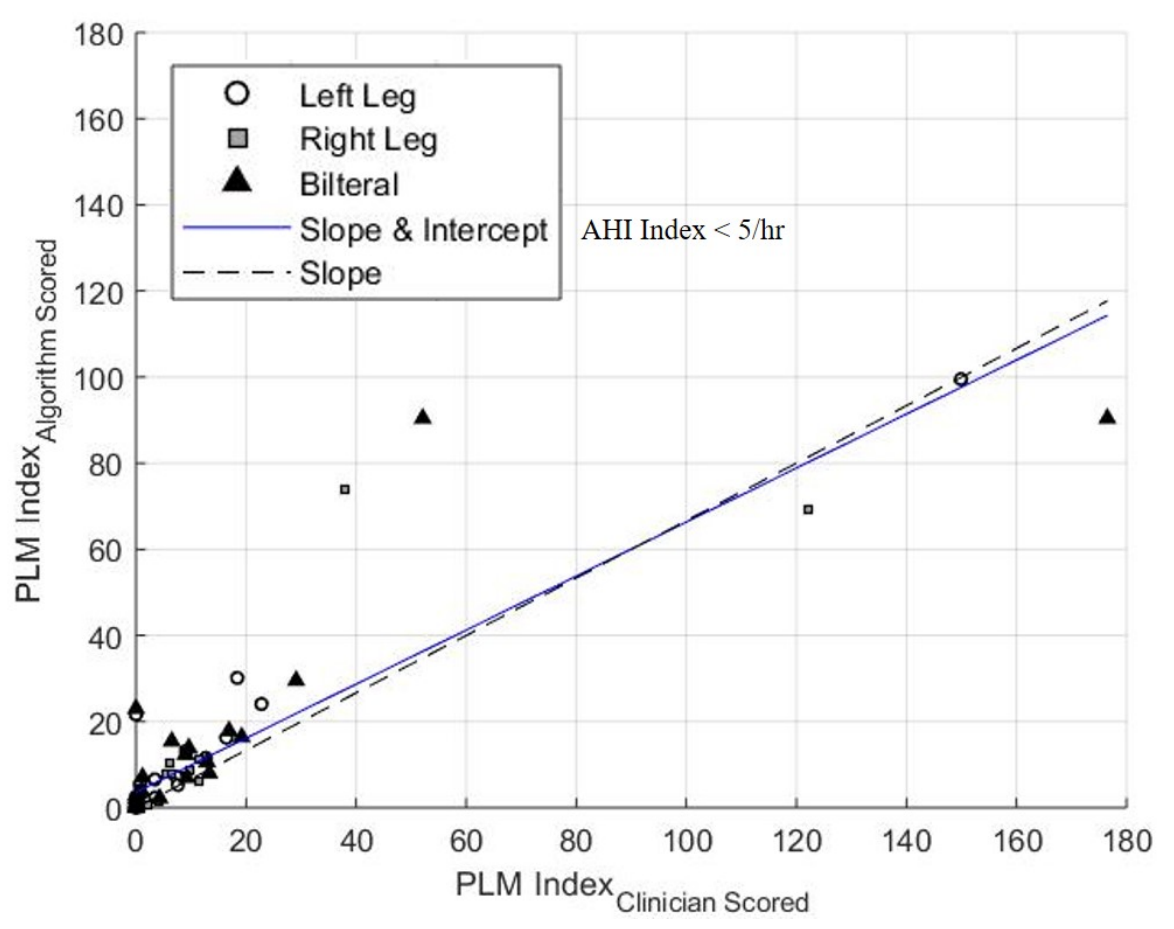

Figure 3.12: Dataset B - Clinical vs. Algorithm scores for 27 (out of 38) subjects $(\mathrm{AHI}<5$ )

normal distribution. This, as reviewed in [36], is one among the few other conditions that needs to be satisfied in order to make inferences using Pearson correlation. Besides, other than being a non-parametric method, Spearman correlation also seems to be more resistant to outliers compared to Pearson correlation, at least when there are not too many of them [37].

Moving on to the results, for the individuals with AHI less than 5 from dataset A, the Spearman correlation coefficient was found to be 0.78 . With regards to dataset $\mathrm{B}$, as hinted above, the Spearman correlation coefficients were similar regardless of whether the scores from the 2 subjects that were identified as outliers were excluded or not. To be exact, with the inclusion of the scores from the 2 outlier subjects, the Spearman correlation coefficient was found to be 0.84 , while without the like, it was found to be 0.80 . Nonetheless, for both datasets, obtaining coefficient values closer to 1 seemed to signify a strong positive correlation [35] between the algorithm and clinical scores. Moreover, it is noted here that all the Spearman correlation coefficients reported thus far 


\section{Sleep Apnea}

AHI Index $<5 / \mathrm{hr}$

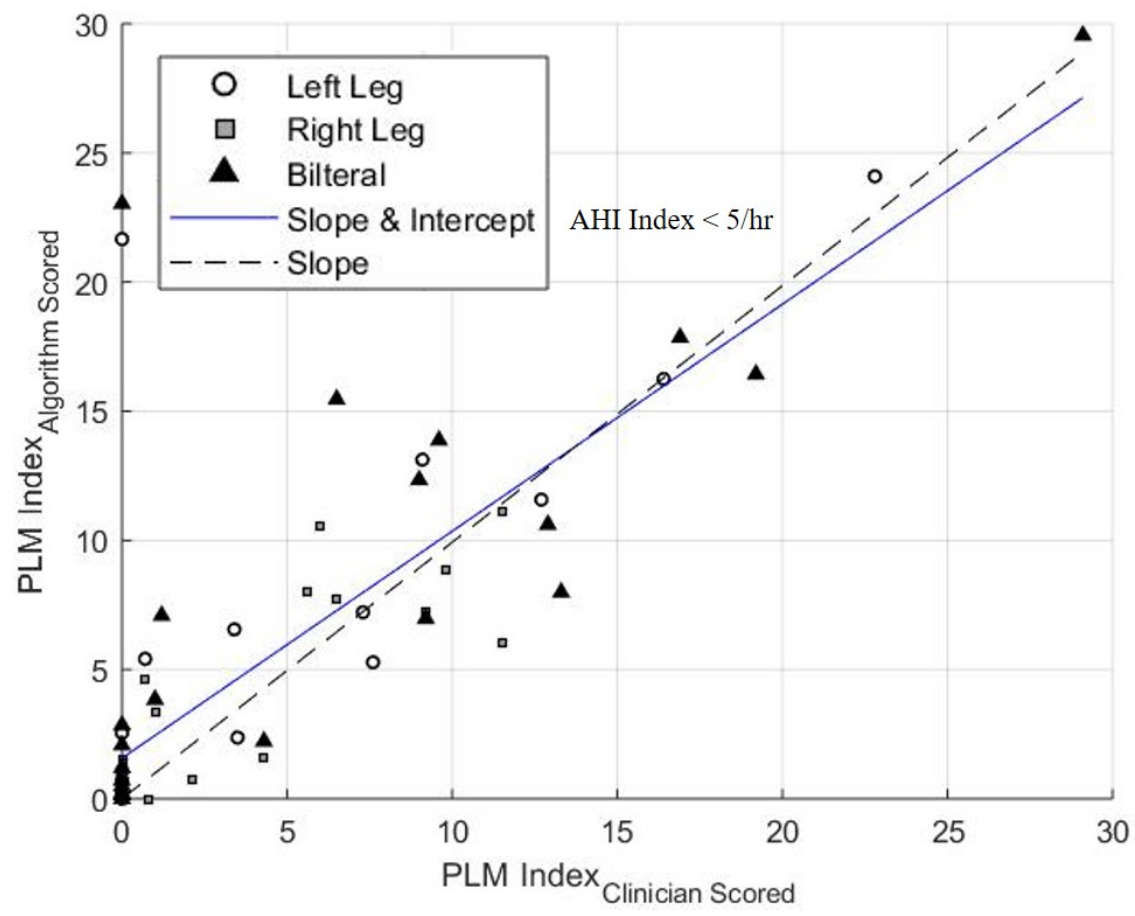

Figure 3.13: Dataset B - Clinical vs. Algorithm scores for 25 (out of 38) subjects $(\mathrm{AHI}<5)$ not including scores from 2 subjects (outliers)

were statistically significant $(\mathrm{p}<0.05)$.

\section{Linear Regression - Slope with or without y-intercept}

More often than not, correlation calculations are accompanied by linear regression analyses [38]. These are depicted here by the equations 3.3 to 3.8. Specifically, for dataset A, for the individuals with AHI less than 5 (refer to Fig. 3.11), the equations for the line with y-intercept and without (using MATLAB's polyfit() [39] function and 'Y' operator, respectively) were:

$$
\begin{gathered}
y=0.82 x+1.82 \\
y=0.90 x
\end{gathered}
$$


Similarly, for the individuals with AHI less than 5 from dataset B, while the respective equations with the inclusion of scores from the 2 subjects deemed as outliers (refer to Fig. 3.12) were:

$$
\begin{gathered}
y=0.63 x+3.63 \\
y=0.67 x,
\end{gathered}
$$

the corresponding equations, without the inclusion of scores from the 2 outlier subjects (refer to Fig. 3.13), were:

$$
\begin{gathered}
y=0.88 x+1.57 \\
y=0.99 x
\end{gathered}
$$

Note that, for both the datasets, as shown above, the equation for the line of equality, i.e., the line without y-intercept was provided as well. This was to be able to decipher, even if just at the basic level, the 'degree of agreement' [40] between the 2 sets of scores under study [40]. In particular, the latter is expanded more upon in the next sub-section.

Nevertheless, while the results from dataset A seemed to lean towards the possibility of a linear relationship between the 2 sets of scores, the results from dataset $\mathrm{B}$, with the inclusion of the scores from the outlier subjects, seemed to do so to a lesser extent. That being said, if the scores from the outlier subjects were not included, Dataset B's results were analogous to that of Dataset A.

To elaborate on the latter, unlike Spearman correlation [37], it is not uncommon for the linear regression parameters to be severely affected by the presence of even just two outliers [41], hence, the reason that for dataset $B$, the slopes with and without the inclusion of the scores from the outlier subjects were as indicated. This brings us to the next sub-section in which, a more appropriate method for comparing 2 sets of scores is introduced.

\section{Bland-Altman}


Specifically, although it is common practice to present the correlation and linear regression results in studies examining automated vs. clinical scores (for e.g. [30]), the Bland-Altman's plot, which is also provided in these studies, is a more suitable approach for such kind of analyses [38], [40]. Explicitly, the latter characterizes the agreement between two sets of measurements being compared by plotting the exact differences between the two against their corresponding means [40]. Moreover, in the same plot, to show the spread of the differences between the two sets of measurements, the mean and the values that are \pm 2 standard deviation away from the mean are typically shown. That being said, these parameters are only valid if the values for the differences between the two measurements being compared follow a normal distribution [40]. In fact, in the current study, the differences between the algorithm and clinical scores did not strictly follow a normal distribution. Hence, instead of mean and standard deviation, non-parametric references, such as the 5th, 50th (median), and 95th percentile values, are depicted in the illustrations that follow [40]. Specifically, shown in Figures 3.14, 3.15, and 3.16, are the Bland-Altman representations of the scores from datasets A and B. Note that, for dataset B, the scores both with and without the inclusion of the two extreme outlier subjects identified earlier are illustrated. That being said, as depicted in the figures, while the median for dataset $\mathrm{A}$ was 0.78 , for dataset $\mathrm{B}$, regardless of whether the outlier subjects were included or not, the median was 0 . To elaborate, this meant that, for at least half of the values in dataset $\mathrm{A}$, the differences between the algorithm and clinical scores were above or below the ' 0.78 ' mark, or in the case of dataset B, they were above or below the ' 0 ' mark. Likewise, while the differences between $90 \%$ of the scores in Dataset A fell between the limits of -9.04 and 6.48, for Dataset B, with the inclusion of the extreme outliers, the limits were -5.37 and 16.21, and without the like, they were -2.61 and 5.6, respectively. Although it would have been ideal to have had a more objective measure of how different from the gold standard the algorithm score should be before reaching unacceptable limits [38], [40], a score only higher or lower than an absolute maximum of 9 from the clinical score for a majority of the data, i.e., not including the extreme outliers, looks promising.

With all that said, it is of interest to speculate on why there might have been extreme outliers for dataset B, in this case, in the first place. One possible reason could have been, and is not limited 


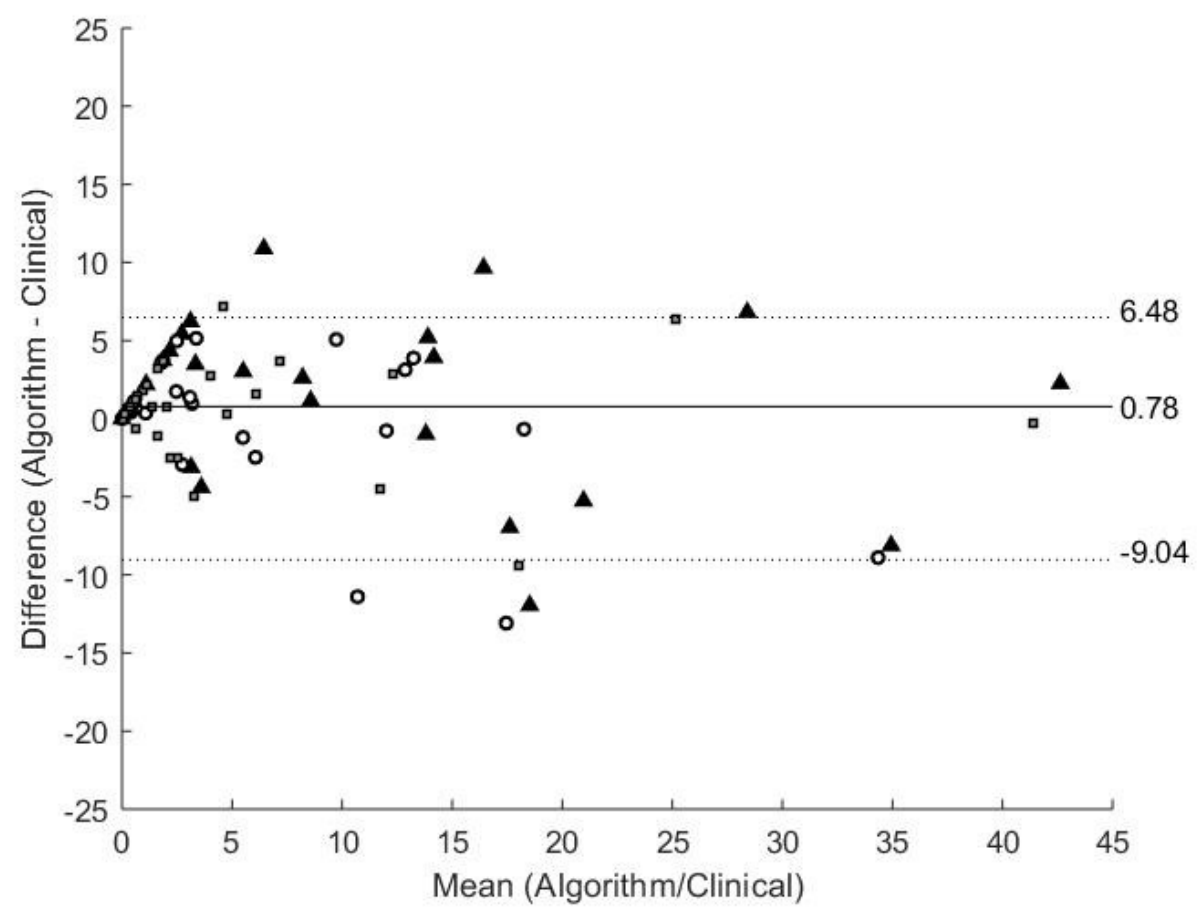

Figure 3.14: Dataset A - Bland Altman plot for 28 (out of 56) subjects (AHI $<5$ )

to the fact that, dynamic changes in the baseline of the signal, perhaps caused due to changes in electrode impedance, or the interference of other artifacts in the signal, as cautioned against in [7], was not dealt with in the algorithm at present. Hence, for a few individuals, for some sections of their EMG, the latter could have resulted in an overestimation or underestimation of PLM due to the picking up of noise as LM, or missing the onsets and offsets of LM, respectively. Moreover, this effect is more pronounced if, for the individual in question, there were more of such sections of EMG. That said, it is expected that for future work, a systematic technique for dealing with such issues arising from dynamic changes to the baseline per segment would be addressed.

Finally, arriving at the end of this section, it is of interest to mention a few words with regards to the individuals with a clinical PLM score of 0. Specifically, for a few of these individuals from this subset, notice the vertical spread of the algorithm scores against the clinical PLM score of 0 (refer to Figures 3.11 and 3.13), or in the case of the Bland-Altman plots, the brief increasing trend in the differences between the scores for the mean of scores less than 5 (refer to Figures 3.14 and 


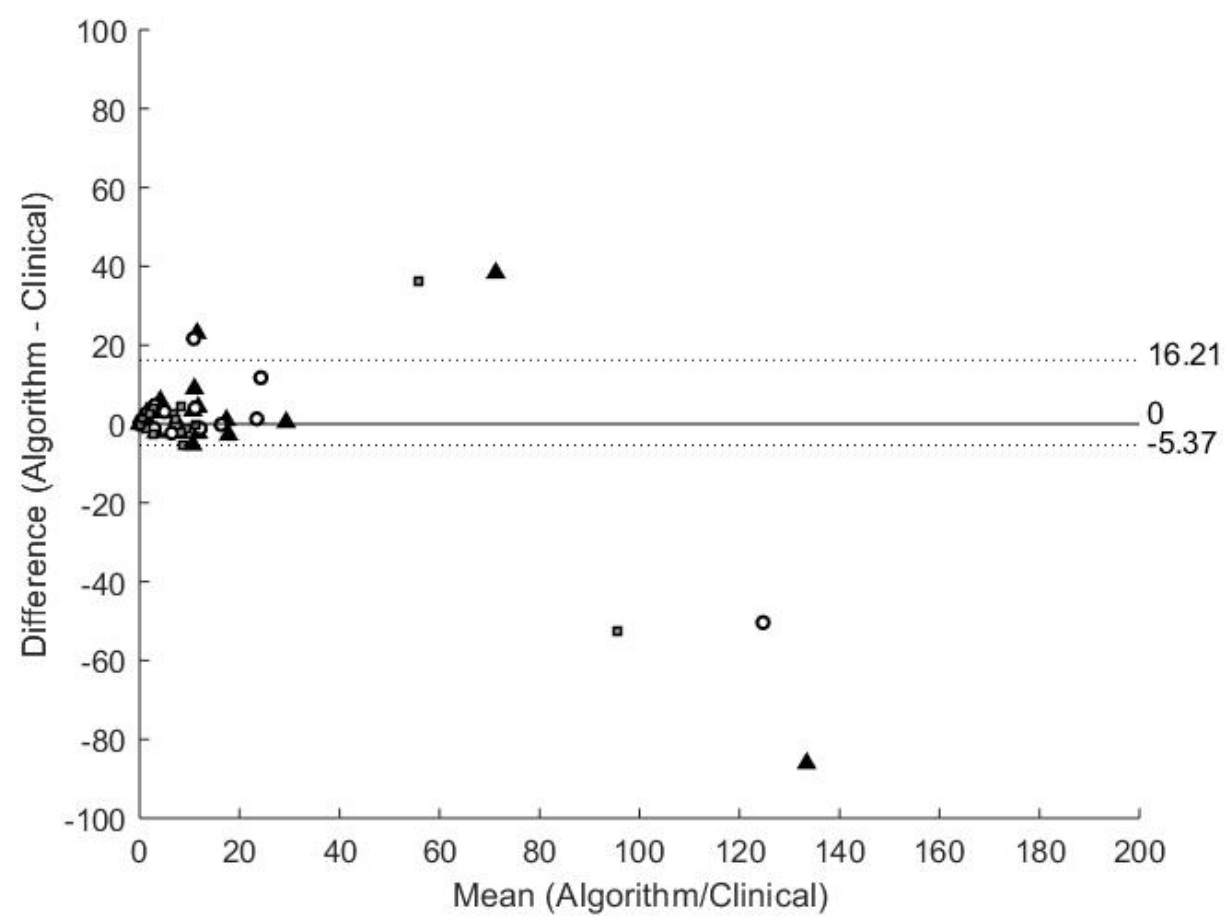

Figure 3.15: Dataset B - Bland Altman plot for 27 (out of 38) subjects (AHI $<5$ )

3.16). As speculated earlier for the individuals with AHI greater than or equal to 5, it is expected that here as well, though thought to be negligible, the inclusion of respiratory-associated PLM in the algorithm scores, otherwise not included in the clinical scores, could have resulted in a higher score for the former over the latter. This is provided that all the PLM that occurred in these individuals occurred parallel to the respiratory events. Nonetheless, for future work, as stated before, the priority would be to isolate the times at which the respiratory events occurred, and then compare the scores calculated this way to the clinical scores.

\subsubsection{Analysis of PLM vs. Sleep Stages}

Using hypnogram data, it was of interest in the current thesis to find out whether out of the total number of PLM that occurred per subject, most of the PLM occurred corresponding to the sleep state, or awake state of the individual in question. This is similar to the concept of scoring for 


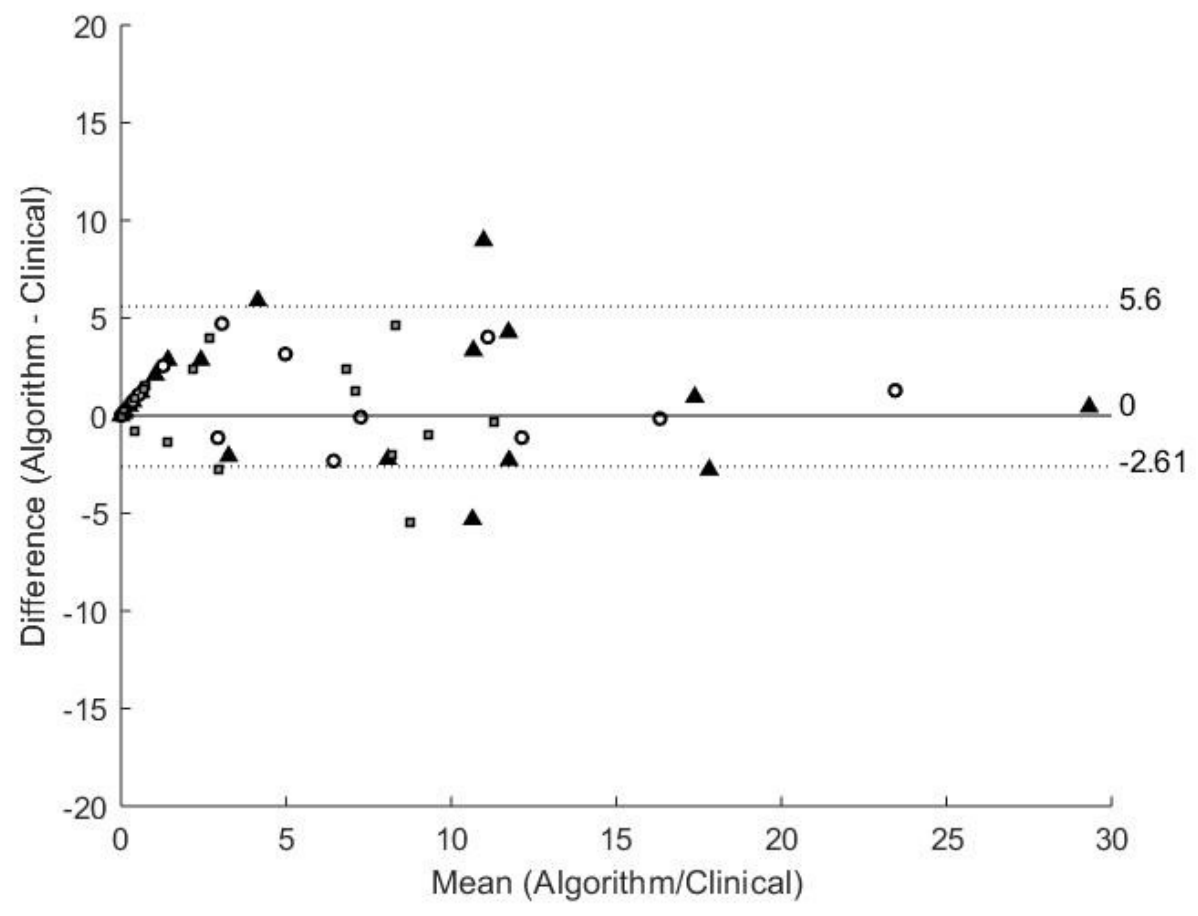

Figure 3.16: Dataset B - Bland Altman plot for 25 (out of 38) subjects (AHI $<5$ ) not including scores from 2 subjects (outliers)

the number of PLM that occurred during sleep or awake, i.e., PLMS or PLMW [7], stated earlier. However, instead of finding out the ratio of the number of PLM that occurred over the respective sleep state by dividing the former over the amount of time spent in that respective sleep state [7], here, it was of interest to find out relatively how much of the portion of PLM that occurred per individual occurred during sleep versus awake. Specifically, this was calculated using the equation 3.9 shown below by replacing the term 'respective state' in the numerator of the ratio in the equation, with 'sleep state' or 'awake state'. For example, if there were 100 PLM that occurred in a subject, if 60 of them occurred during sleep, then the percent of PLM that occurred during sleep for that individual would be $60 \%$, and the percent of PLM that occurred during awake for that individual would be $40 \%$.

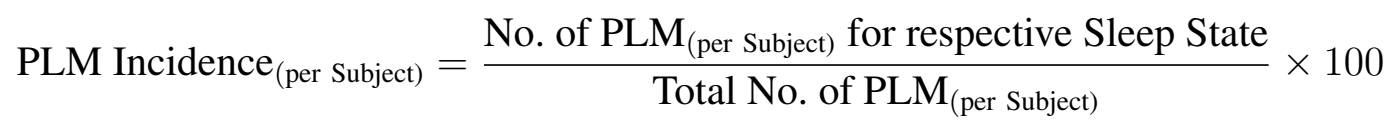


Moreover, not only was the percent of PLM that occurred during sleep versus awake calculated per subject, but also the percent of PLM that occurred during a particular sleep stage for that subject was calculated as well. Namely, the sleep stages considered were non-REM 1, 2, 3, and REM. In particular, in the current thesis, non-REM 1 and 2 were combinedly represented as 'non-REM $1 \& 2$ ' to distinguish them from non-REM 3, which belonged to a deeper stage of sleep [42]. Nonetheless, going back to the example of the 100 PLM occurring for a subject, if, out of the 60 PLM that occurred during sleep, 50 of them occurred during non-REM $1 \& 2,7$ occurred during non-REM 3, and 3 occurred during REM, then the number of PLM that occurred during each of these sleep stages over the total number of PLM that occurred for that subject would be 50\%,7\%, and 3\%, respectively. From the latter, for example, it can be deduced here that for this particular subject, most of the PLM occurred during sleep over awake, and out of the PLM that occurred during sleep, most of them occurred corresponding to non-REM $1 \& 2$.

With that said, the results from calculating the percent of PLM, i.e., termed 'PLM incidence' here, for each sleep state, for all the subjects from dataset A and dataset B, are as shown in Figures 3.17 and 3.18, respectively. Specifically, within the boxplot of each sleep state, the scatter of data represents the PLM incidence value obtained for each individual for that given sleep state.

Moreover, note that the parameters from this analysis were obtained using the bi-lateral PLM only as opposed to the mono-lateral PLM identified per subject. The idea behind the choice of the former over the latter was so as to not unintentionally duplicate the results since, the bi-lateral PLM, as stated before, were found by superimposing or combining where relevant [7], the PLM from both the legs. Lastly, individuals with hardly any PLM detected, i.e., 1 subject from dataset A and 2 from dataset B, were the only ones not included in this analysis. Regardless, shown below are the key findings from this analysis:

- PLM incidence seemed to be significantly higher $(\mathrm{p}<0.05)$ during sleep compared to awake periods. This is in contrast to the findings by [28], [30] in which, the PLMW scores, or in other cases, the count of PLM that occurred during awake, was found to be higher than the PLMS scores, or the count of PLM that occurred during sleep, respectively. It is speculated here that, perhaps having a dataset comprising primarily of individuals with RLS over 

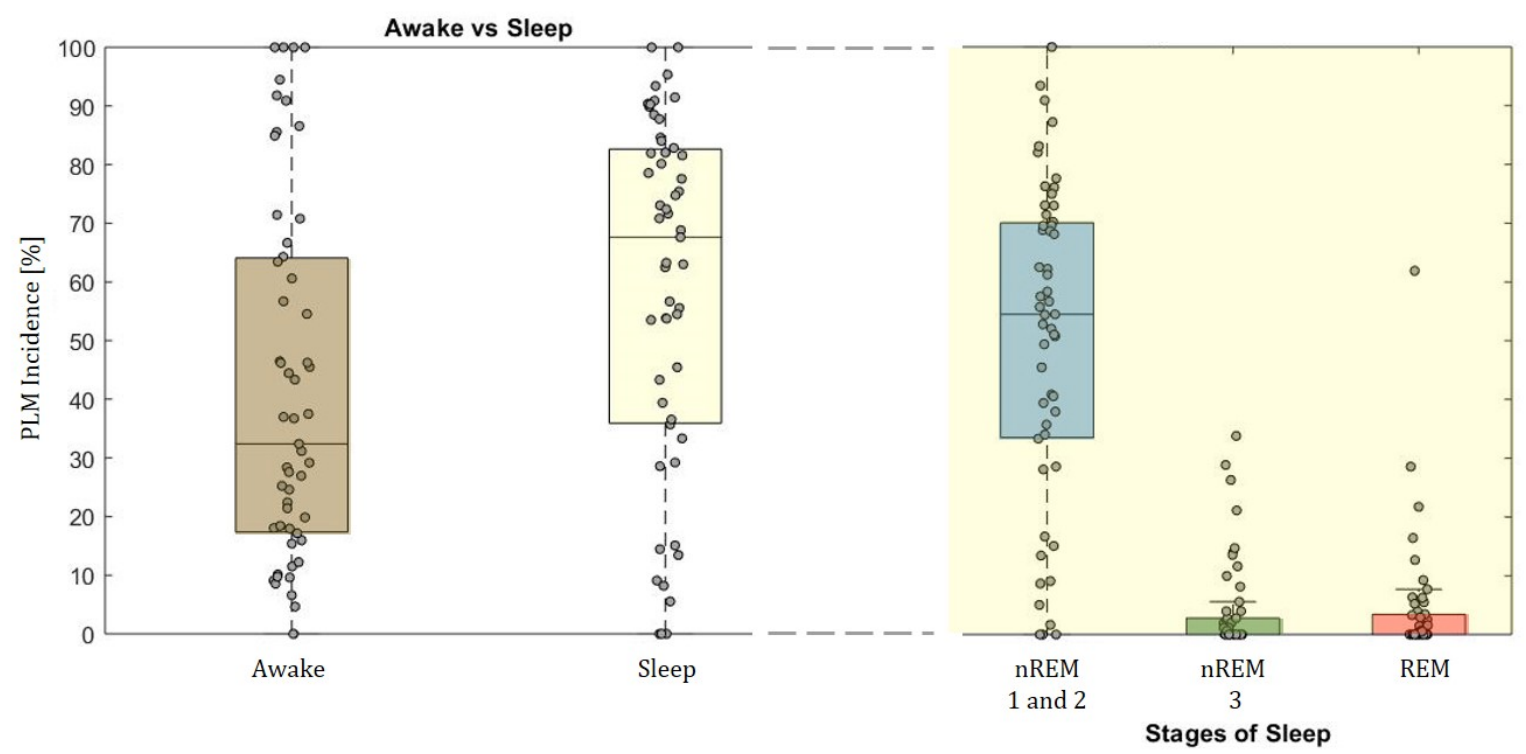

Figure 3.17: Dataset A - PLM Incidence with respect to the different sleep states

PLMS, as was the case in [28], [30], could alter the likelihood of finding PLM during sleep over awake times.

- Out of the PLM that occurred during sleep, PLM incidence seemed to be significantly higher $(\mathrm{p}<0.05)$ during non-REM $1 \& 2$ compared to non-REM 3 or REM. This is analogous to the findings of such studies like [43]. Furthermore, it is noted here that, while most studies [28], [30] looked at the PLM count or score occurring per non-REM stage overall, only a few others like [43] distinguished between PLM that occurred during non-REM 1, 2, and 3 .

Nevertheless, as a whole, the results presented in this section adds to past studies' results [28], [30], [43] that, PLM, for the most part, is more likely to be captured during the non-REM part of sleep, i.e., particularly during non-REM 1 and 2. Hence, from a monitoring perspective, the latter identifies, based on sleep stages, the regions wherein PLM are most likely to occur in a long-term 


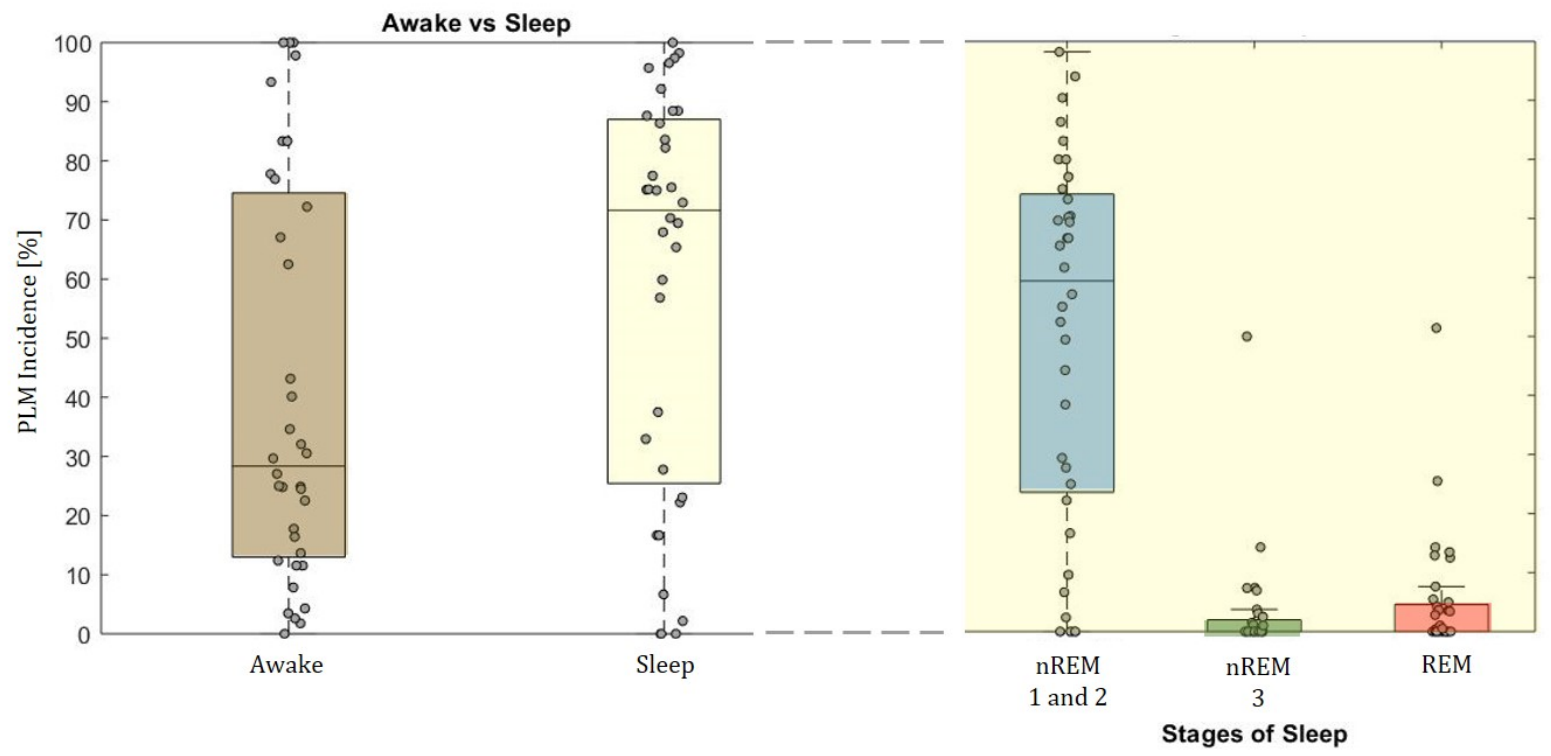

Figure 3.18: Dataset B - PLM Incidence with respect to the different sleep states

signal. On the other hand, since a notable portion of PLM that occurred per individual also seemed to occur during the awake state, though to a lesser extent than that of during their sleep state, it was of interest to look into the differences or similarities of the dimensions of these PLM that occurred during awake compared to those that occurred during sleep. Moreover, given that most studies seem to look into the characteristics of LM across sleep overall rather than across the different stages of sleep, it was of interest here to carry forth this analysis across the different stages of sleep in order to gain a better understanding of the PLM phenomenon. That being said, this brings us to the next section in which, precisely, the LM, or IMI duration, of PLM that occurred during awake, sleep, and the individual sleep stages, are analyzed.

\subsubsection{Analysis of LM and IMI Duration of PLM per Sleep Stage}

Before going through the specifics of the analysis in this section, it is significant to mention that, here as well, only the results obtained using bi-lateral PLM as opposed to mono-lateral PLM are 
shown. That being said, given the range of possible values for LM and IMI durations, i.e., 0.5 to $15 \mathrm{~s}$ and 10 to 90 s respectively [7], it was of interest here to construe the relative frequency distribution of these 2 parameters within each given state of sleep, for each individual being analyzed. To be exact, in order to achieve this, the equations 3.10 and 3.11 shown below, pertaining to the total PLM found corresponding to a given sleep state (for each subject), were used:

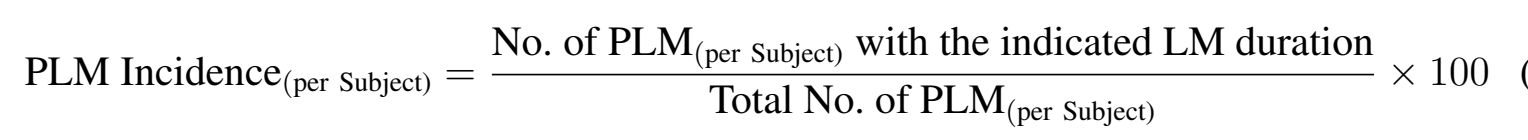

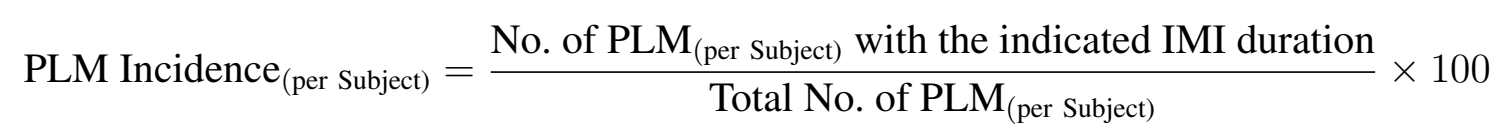

To illustrate the above, say a subject had a total of 100 PLM that occurred corresponding to the awake state, and if 40, 35, and 25 of each of those LM lasted within the ranges of $0.5 \leq t_{1}<1 \mathrm{~s}$, $1 \leq t_{1}<1.5 \mathrm{~s}$, and $1.5 \leq t_{1}<2 \mathrm{~s}$ respectively, then, it can be deduced from the latter that, out of all the PLM that occurred during the awake period for this individual, most (40\%) of them had individual LM durations of 0.5 to $1 \mathrm{~s}$, and the rest either had individual LM durations of 1 to $1.5 \mathrm{~s}$ (35\%) or 1.5 to $2 \mathrm{~s}(25 \%)$. Likewise, if out of the 100 PLM that occurred corresponding to awake time, 50, 30, and 20 of each of those LM occurred within the ranges of $10 \leq t_{2}<15 \mathrm{~s}, 15 \leq t_{2}$ $<20$ s, and $20 \leq t_{2}<25$ s respectively from the previous LM, then, it can be deduced here that, out of all the PLM that occurred during the awake period for this individual, most (50\%) of them had individual IMI durations of 10 to $15 \mathrm{~s}$, and the rest either had individual IMI durations of 15 to $20 \mathrm{~s}(30 \%)$ or 20 to $25 \mathrm{~s}(20 \%)$. As a side note, $t_{1}$ and $t_{2}$ here represent the LM duration and IMI duration, respectively. Notwithstanding, to find the relative frequency distribution of these 2 parameters for each of the other sleep states of this individual, the above would have to be repeated for the remaining PLM found corresponding to the other sleep states in this individual.

With all that said, the results from implementing all of the above on the individuals from datasets A and B, are as shown in Figures 3.19 to 3.26. In essence, similar to the previous section, 
within the boxplot of each LM or IMI duration range for the sleep state being analyzed, the scatter of data represents each subject's percent of PLM satisfying the specified range of LM or IMI duration within the respective sleep state. Moreover, like in the previous section, only the subjects who had PLM within whichever state of sleep being analyzed were included in this analysis. This is reflected by the $\mathrm{N}$ number provided in the top right corner within each of the distributions shown.

Nevertheless, with regards to the key observations from the 'LM duration' analysis:

- For most of the individuals who had PLM during the awake state (refer to Figures 3.19 and 3.20), the LM seemed to most frequently last between 0.5 to $1 \mathrm{~s}$, although this was less the case for dataset B. Nonetheless, this was before the former tapered off towards the end of the distribution where, the least likely LM duration was anywhere between 10 to $15 \mathrm{~s}$.

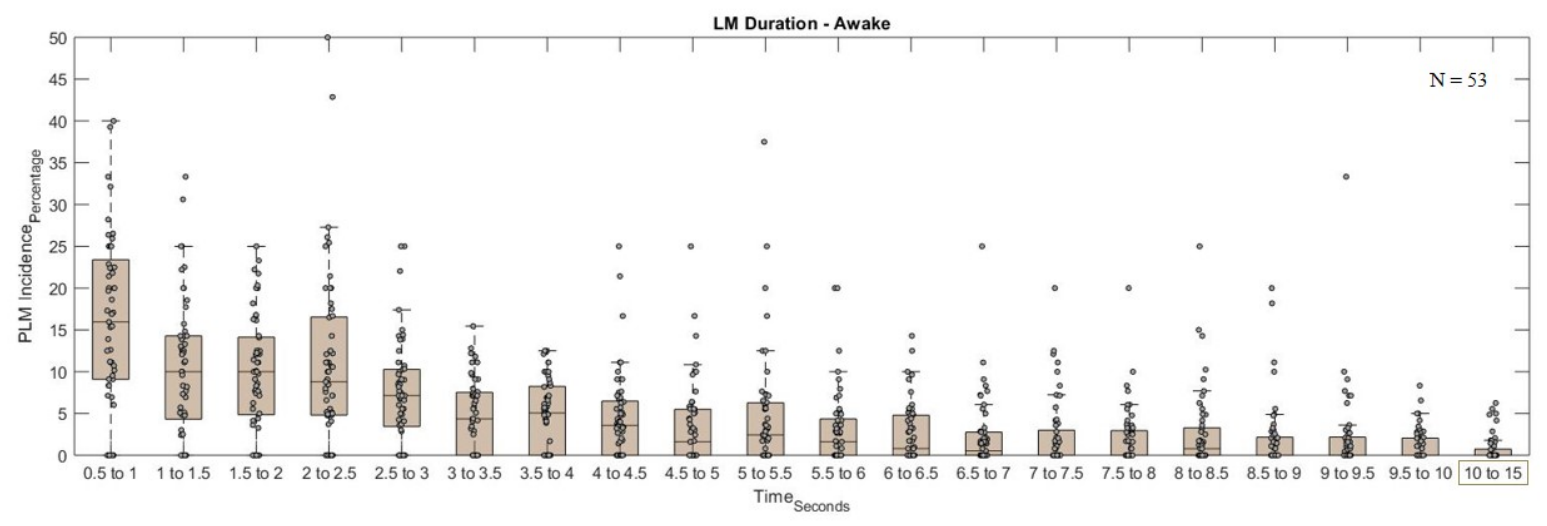

Subjects who did not have PLM during Awake Time were discarded.

Figure 3.19: Dataset A - LM duration distribution of PLM occurring during the awake state

- In fact, the decreasing trend observed above appeared to be more pronounced and skewed during the REM state of sleep (refer to the 4th subplots from the Figures 3.21 and 3.22) where, although there were fewer individuals with PLM that occurred corresponding to this sleep stage to start with, for these few individuals, the LM most frequently lasted between 


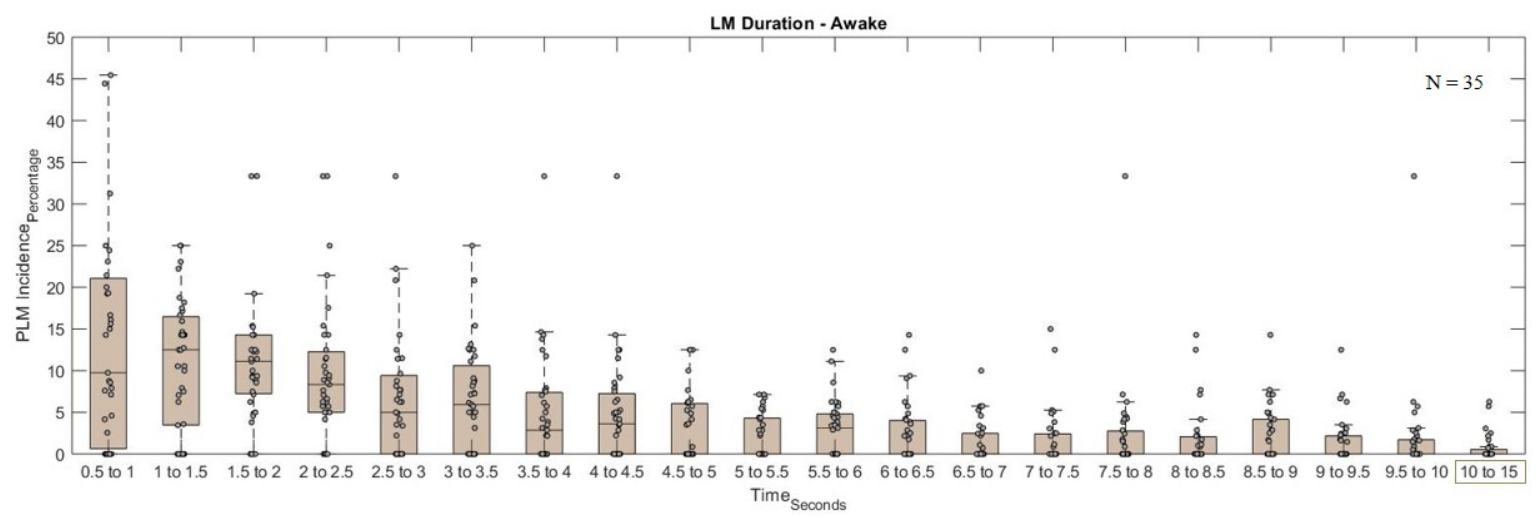

Subjects who did not have PLM during Awake Time were discarded.

Figure 3.20: Dataset B - LM duration distribution of PLM occurring during the awake state

0.5 to $1 \mathrm{~s}$ before tapering off towards a limit of $3.5 \mathrm{~s}$ beyond which, hardly any PLM existed.

- That being said, during sleep and non-REM $1 \& 2$ in particular (refer to the 1 st and 2 nd subplots of Figures 3.21 and 3.22), more frequently than not, for most of the individuals here, their PLM seemed to last longer, i.e., around 1 to $1.5 \mathrm{~s}$ (for Dataset A), and 1.5 to 2s (for Dataset B), before tapering off towards a limit of $6.5 \mathrm{~s}$ beyond which, hardly any PLM existed. Note that, the similar result for sleep and non-REM $1 \& 2$ is not unexpected since, as concluded from the results from the previous section, as well as found in past studies [28], [43], the PLM that occur during sleep predominantly seemed to belong to the non-REM 1 \& 2 stages of sleep.

- Aside from all that, for the few PLM that occurred corresponding to non-REM 3 (refer to the 3rd subplots of Figures 3.21 and 3.22), the frequency distribution for the LM duration seemed to be more disorderly compared to that of the PLM from any of the other sleep stages. Specifically, in this case, the LM lasted from anywhere between 0.5 to $4 \mathrm{~s}$. 


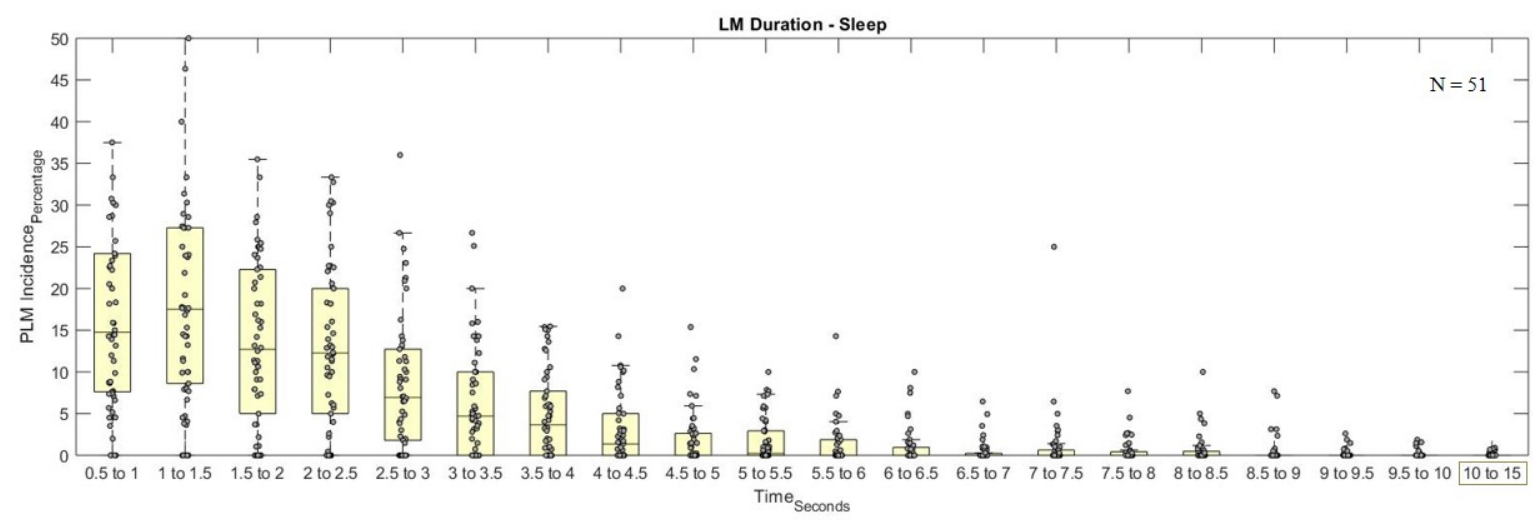

Subjects who did not have PLM during Sleep Time were discarded.
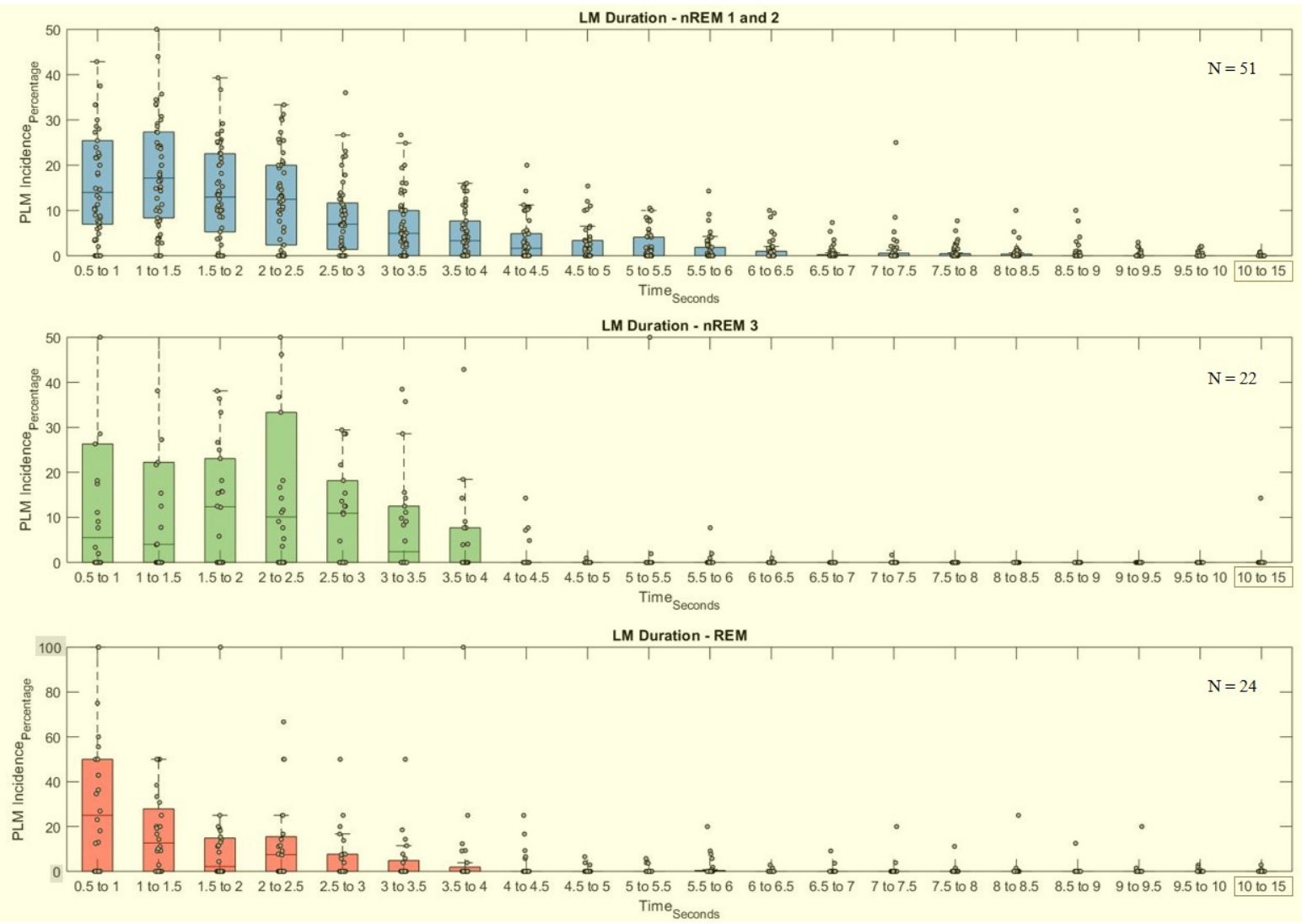

Subjects who did not have PLM during the respective Sleep Stage were discarded.

Figure 3.21: Dataset A - LM duration distribution of PLM occurring during other sleep states 


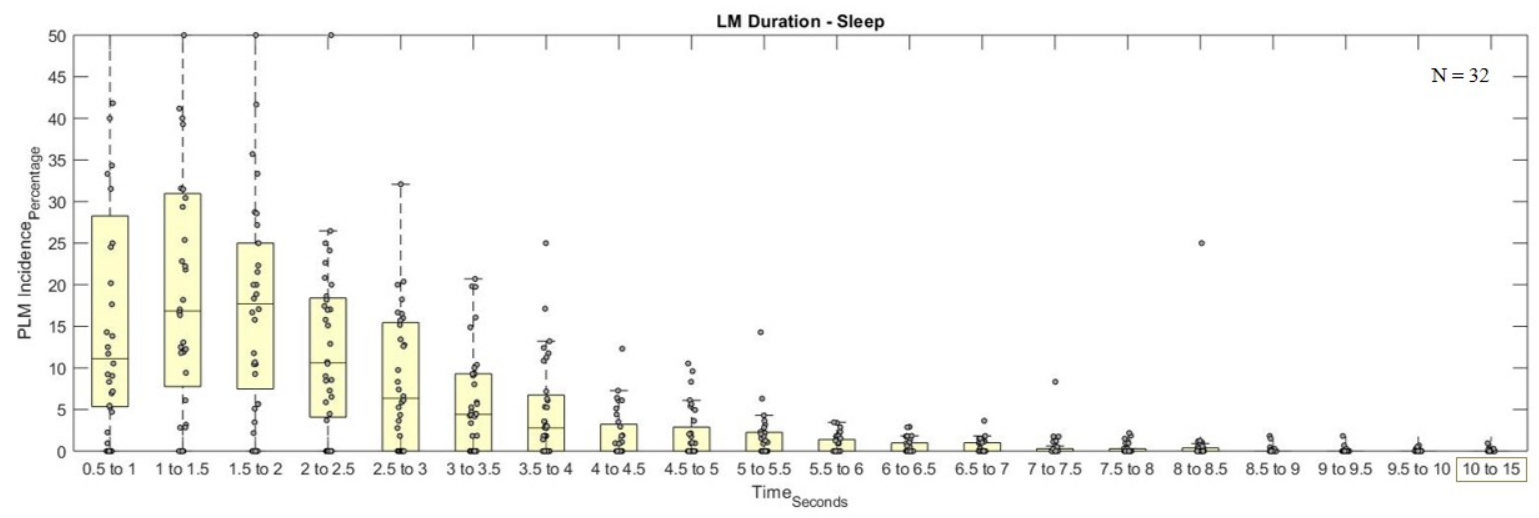

Subjects who did not have PLM during Sleep Time were discarded.
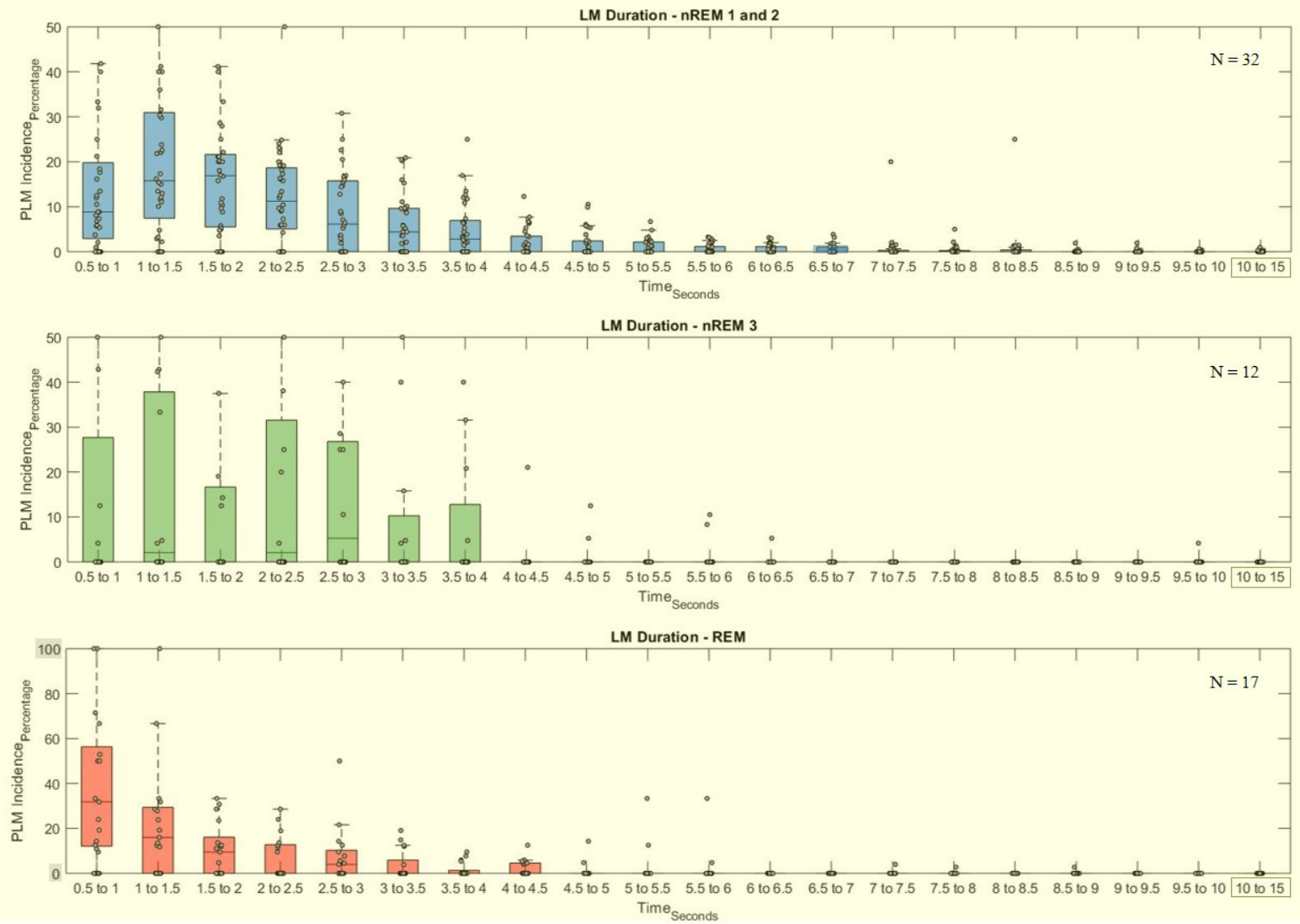

Subjects who did not have PLM during the respective Sleep Stage were discarded.

Figure 3.22: Dataset B - LM duration distribution of PLM occurring during other sleep states 
With regards to the key observations from the 'IMI duration' analysis:

- For most of the individuals who had PLM during the awake state (refer to Figures 3.23 and 3.24), the LM for these individuals seemed to most frequently occur within 10 to 20 s from the previous LM before gradually tapering off towards the end of the distribution.

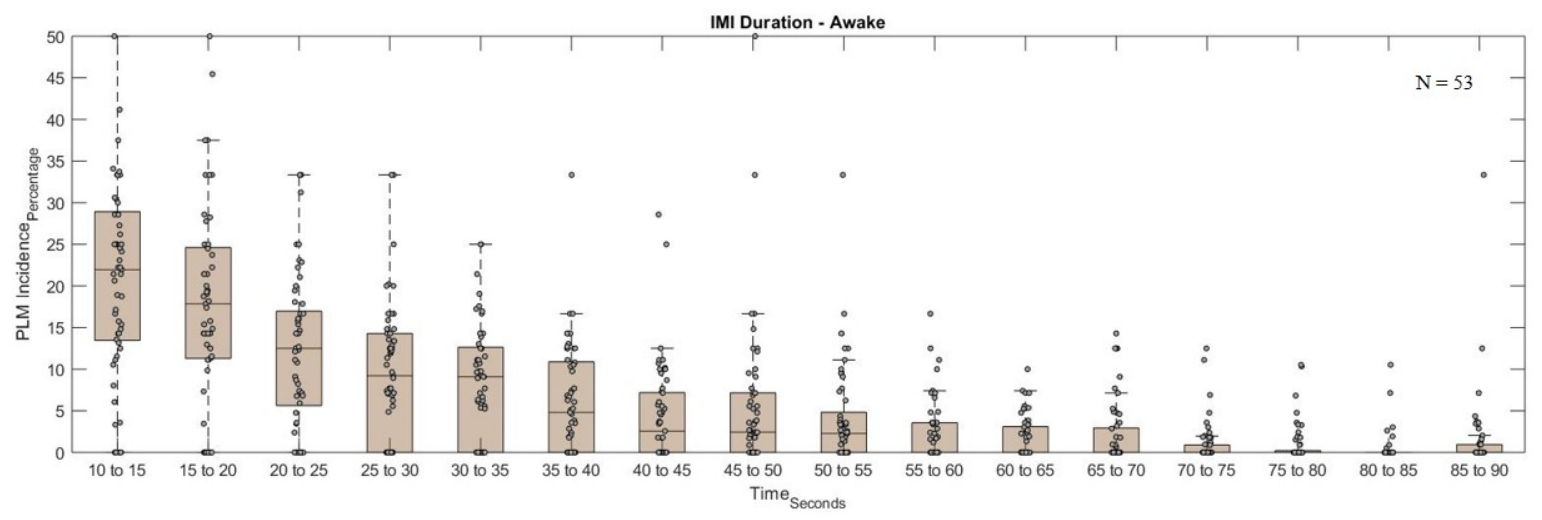

Subjects who did not have PLM during Awake Time were discarded.

Figure 3.23: Dataset A - IMI duration distribution of PLM occurring during the awake state

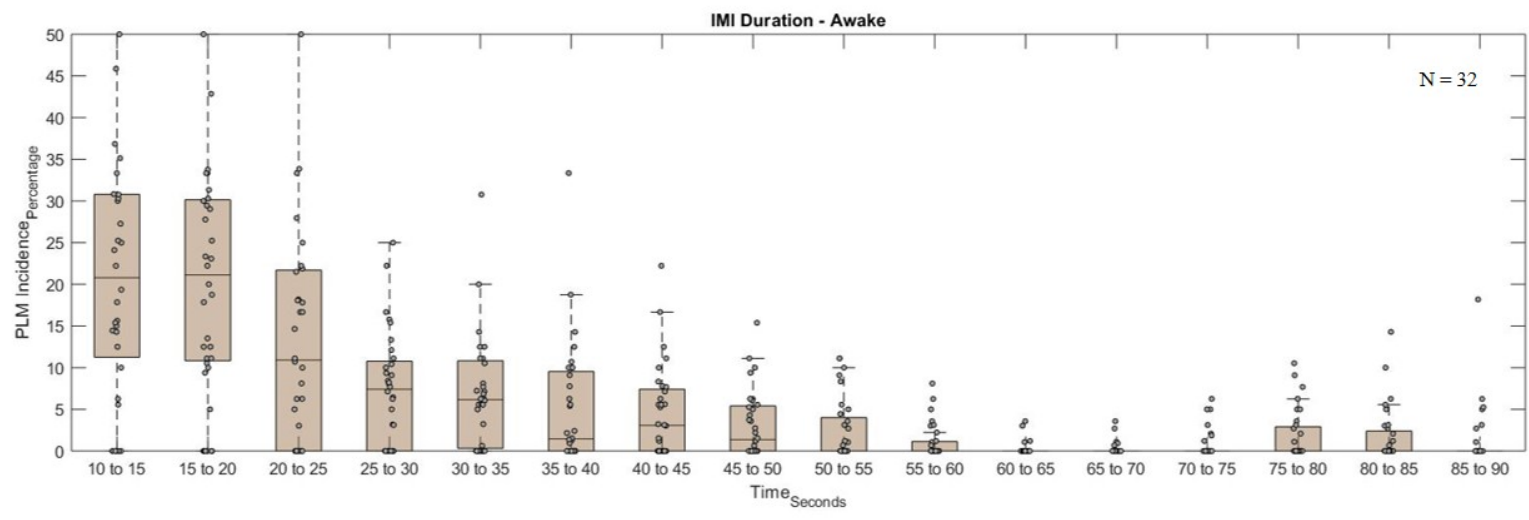

Subjects who did not have PLM during Awake Time were discarded.

Figure 3.24: Dataset B - IMI duration distribution of PLM occurring during the awake state

- This was unlike that of the PLM occurring during sleep and non-REM 1 \& 2 (refer to the 1 st 
and 2nd subplots of Figures 3.25 and 3.26) where, for most of the individuals here, the LM seemed to most frequently occur within 20 to 30 s from the previous LM before gradually tapering off towards the end of the distribution. Note that, here as well, as stated earlier in the 'LM duration' part of the analysis, the similar results for sleep and non-REM $1 \& 2$ was not unexpected since the PLM that occurred during sleep predominantly belonged to the non-REM 1 \& 2 stages of sleep [28], [43].

- With regards to the PLM occurring during the REM stage of sleep (refer to the 4th subplot from the Figures 3.25 and 3.26), the frequency distribution of IMI duration seemed to lack the definitive trend exhibited by the PLM from the other sleep stages stated thus far. Specifically, in this case, while some of the individuals seemed to have IMI duration spanning anywhere between 10 to 70 s, others seemed to occupy the 80 to 90 s range.

- Likewise, for the PLM that occurred corresponding to non-REM 3 (refer to the 3rd subplot from the Figures 3.25 and 3.26), the IMI duration frequency distribution did not seem to occupy a precise range of values either. Specifically, in this case, the LM seemed to occur anywhere between 15 to 65 s from the previous LM.

In summary, the results here seem to suggest that PLM are more short-lived during the awake and REM stages of an individual compared to the non-REM stages of sleep. Specifically, the latter was somewhat consistent with the results from [43] for example, in which, the LM duration was found to be shortest during the REM stages of sleep compared to the non-REM stages of sleep. However, unlike here, [43] determined the LM durations during the awake stages of sleep to be the longest compared to the other stages of sleep.

Nonetheless, with regards to the IMI duration, unlike for that of the awake and non-REM 1 \& 2 stages of sleep, the IMI duration for the PLM occurring during REM and non-REM 3 seemed to occupy a more variable and wider time span. That being said, the latter seemed to be somewhat 


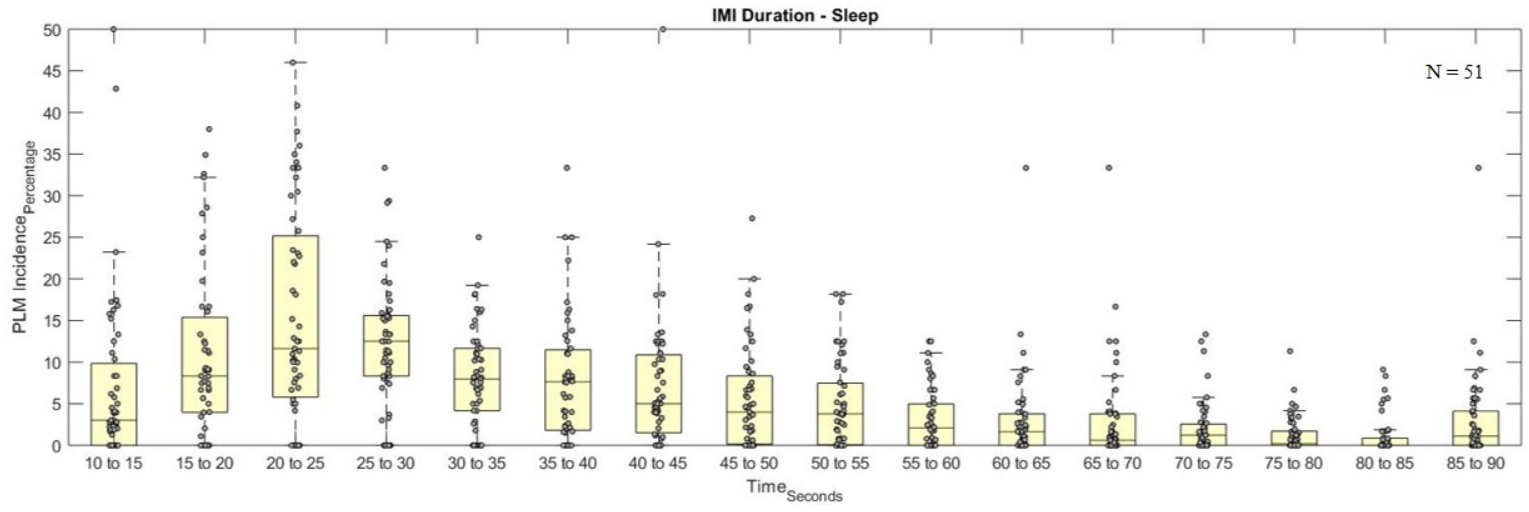

Subjects who did not have PLM during Sleep Time were discarded.
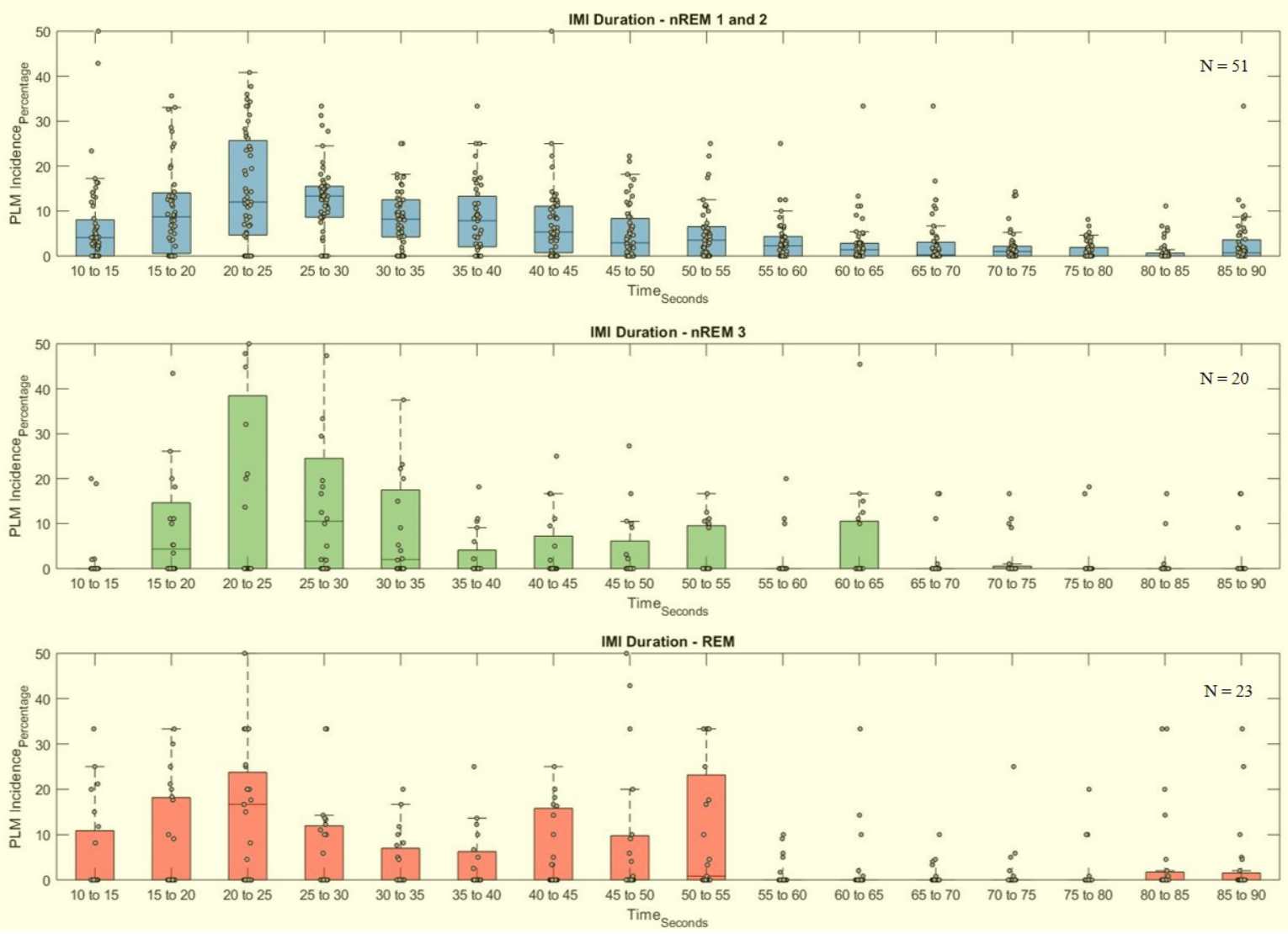

Subjects who did not have PLM during the respective Sleep Stage were discarded.

Figure 3.25: Dataset A - IMI duration distribution of PLM occurring during other sleep states 


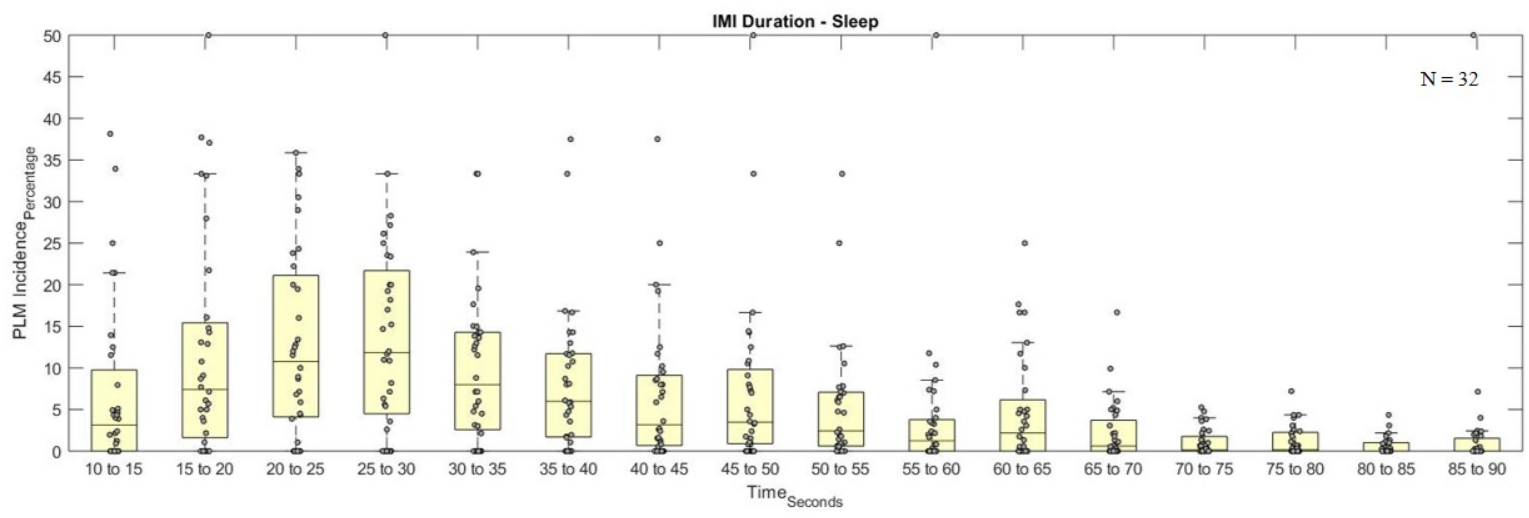

Subjects who did not have PLM during Sleep Time were discarded.
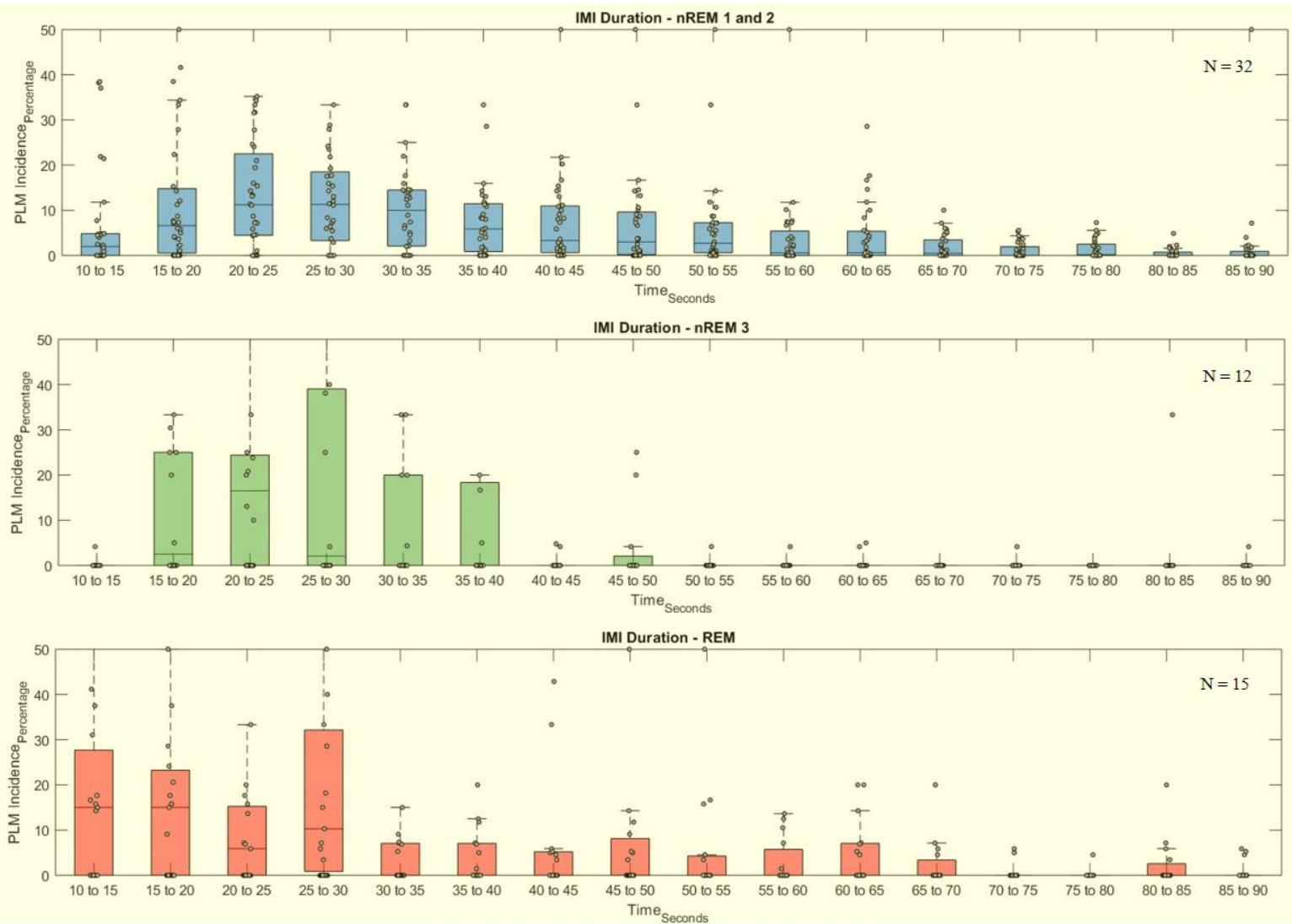

Subjects who did not have PLM during the respective Sleep Stage were discarded.

Figure 3.26: Dataset $B$ - IMI duration distribution of PLM occurring during other sleep states 
consistent with the findings by [43] in which they found the IMI duration to be the longest during the REM stages of sleep compared to the non-REM or awake stages of sleep.

As a whole, as inferred in [43], here as well, it is speculated that the underlying mechanisms during the REM stages of sleep probably inhibit the occurrence of PLM. This is as reflected by the fewer incidence of PLM in this stage, the shorter durations of those LM, and their wider time interval of occurrence from each other [43]. Moreover, although not so much for the deeper stage of sleep, i.e., non-REM 3, the combination of higher PLM incidence, comparatively longer LM durations, and shorter IMI durations during the non-REM $1 \& 2$ stages of sleep seem to suggest the least inhibition of PLM during this stage of sleep, as implied in [43], not including awake though. With that said, the mechanisms underlying PLM in the awake state seem to be more complex since, while the higher incidences of PLM with shorter IMI durations seem to suggest a higher frequency of PLM in this state, the higher incidences of PLM in the shorter LM duration range seem to suggest otherwise. Nevertheless, it is concluded here that further analyses with a much larger and more representative dataset is needed before making definite interpretations with regards to the latter.

\subsection{Summary}

Overall, in this Chapter, an alternative automated PLM detection method, based on [7], was introduced. In particular, a step-by-step implementation of the latter was provided. Moreover, the results from testing out the algorithm on actual datasets were provided, or more precisely, an indepth comparison of the scores obtained from the algorithm with that of those obtained from the clinicians was made. Finally, PLM incidence, and their dimensions, i.e., LM and IMI durations to be exact, with respect to the different sleep stages were explored via relative frequency distributions towards the end of the Chapter. In essence, the implications of the results from this Chapter are reviewed in Chapter 5. That being said, this brings us to the next Chapter in which, the PLM segmentation obtained per subject here, is used to study the differences in HR with respect to PLM for the individuals with or without PLM. 


\section{Chapter 4}

\section{Analysis of PLMS in relation to HR Signals}

Typically, supervised classification [44], in the simplest of terms, involves using features from a signal or a set of signals obtained from a subset of individuals, and being able to predict using the latter, whether an untrained individual for example, can be labeled as being abnormal or normal, or in this case, as having PLM or not. Nonetheless, in the current chapter, instead of designating a subject as being abnormal or normal, given that the exact time stamps of PLM occurrences per subject was known, as acquired from the previous chapter, it was of interest here to be able to discriminate between PLM vs. non-PLM periods occurring within a given subject. Concurrently, to facilitate long-term monitoring of PLM, it was also of interest here to decipher whether the latter could be achieved using features extracted from easily attainable signals such as HR signals, for example, derived from ECG signals. In essence, the idea here, was to find out, whether solely based on certain features from the HR signal, it is possible to distinguish between abnormal and normal, i.e., PLM and non-PLM periods for a given subject. In other words, this translates to being able to classify, from the HR signal (obtained from the heart), the occurrences of PLM (happening at the level of the limbs) within a subject. Indeed, as discussed in Chapter 2, the latter is completely based on the hypothesis [15] and results from past studies [23], [24] that, the autonomic nervous system, i.e., reflected in HR signals, is somewhat associated with the occurrence of PLM. Likewise, as mentioned in Chapter 1, also of interest in the current chapter was to be able to decipher whether, given the PLM information, the HR features extracted could provide clinicians with more feasible means of tracking the changes in HR in response to medications that are provided to treat PLM 
(over successive sessions). In other words, provided that the PLM-CVD link [4] holds true, it is anticipated that the latter would be beneficial for intervention studies that look into the possibility of preventing CVD by treating PLM [12].

That being said, the current chapter is subdivided into two major sections such that, in the first part of the chapter, the steps involved in obtaining the HR information from sleep-derived ECG signals, the challenges involved in processing such signals, and the measures taken to overcome the latter, are discussed. For the remaining part of the chapter, while one portion elaborates on what the relevant features extracted from the processed HR signal were, the other half touches upon on how those features were obtained and used to differentiate between PLM and non-PLM periods occurring within a given subject. Moreover, it is of interest to mention here that, for all of the above, the signals from the same Datasets A and B, used earlier in chapter 3, were used here as well. Nonetheless, Figure 4.1, summarizes all the steps involved from HR processing to classification, each of which, is thoroughly discussed as the chapter unfolds. In fact, in order to obtain a better understanding of the figure, the reader is advised to refer back to it at the end of the chapter.

\subsection{ECG Pre-processing}

\subsubsection{R Peak detection and HR Signal acquisition}

To start with, the very first step from the entire process as depicted in Figure 4.2 involves detecting $\mathrm{R}$ peaks from a given ECG signal. In order to do so, it is of interest to mention here that, the well-known Pan Tompkins technique, founded by [45], was used. In fact, to be exact, the software implementation of the latter, i.e., in MATLAB, developed by [46], was used. For example, shown in Figure 4.2 is a short sample of an ECG signal taken from a subject belonging to the datasets (A and B) mentioned earlier. Note how, shown within the same figure (in the 2nd subplot), are the locations of the $\mathrm{R}$ peaks detected using the Pan-Tompkins technique [45] implemented using the software by [46]. Moreover, analogous to the latter, also shown (in Figure 4.3), is the corresponding 


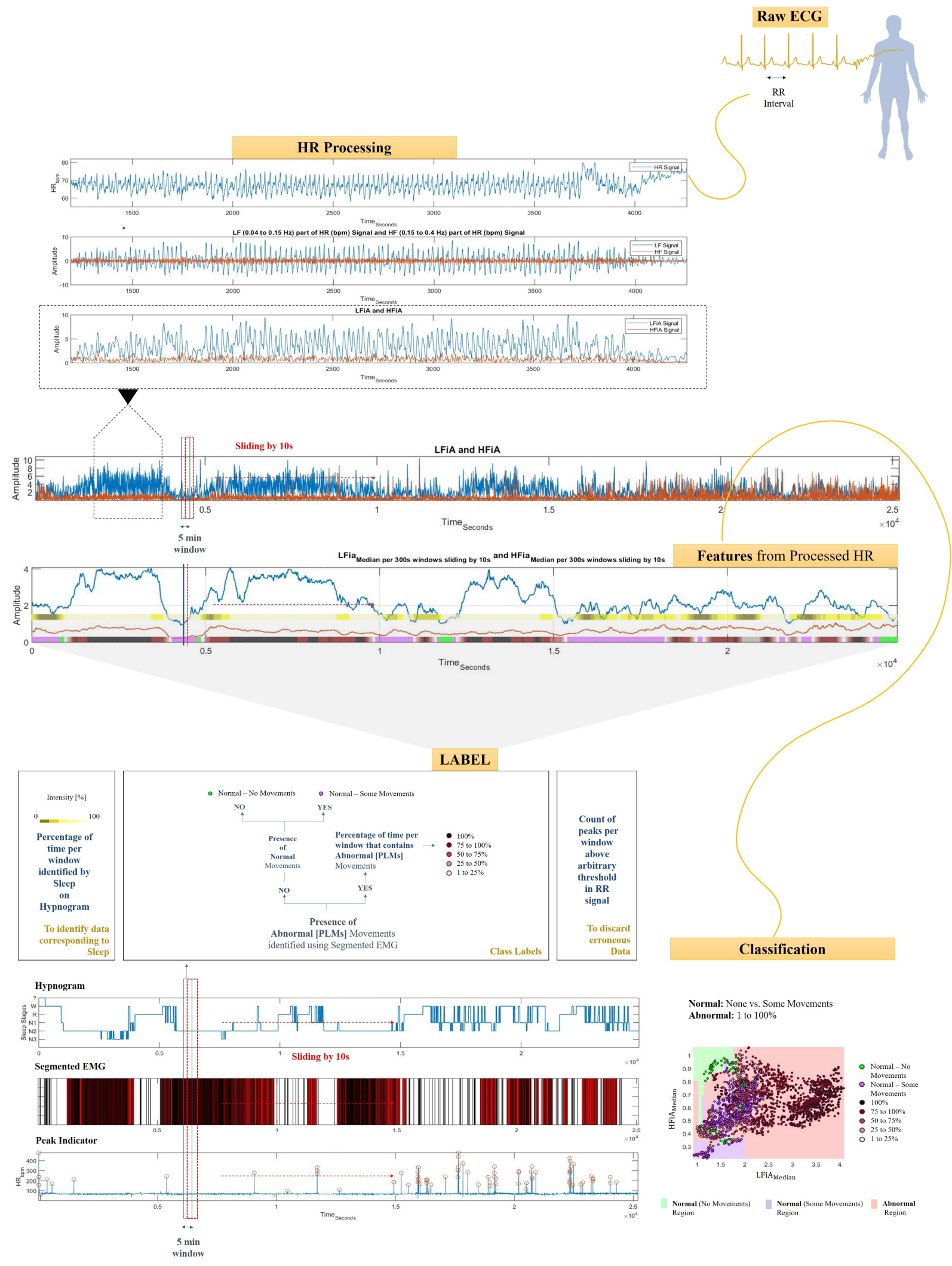

Figure 4.1: Overview of Chapter 4 
HR values. In particular, the latter was calculated using [47]:

$$
H R_{(i)}=\frac{1}{R R_{(i)}} \times 60
$$

where, $H R_{(i)}$ represents the HR at the location of the $i^{\text {th }} \mathrm{R}$ peak, and $R R_{(i)}$, represents the RR interval at the $i^{t h} \mathrm{R}$ peak. Specifically, the latter is given by:

$$
R R_{(i)}=R_{(i)}-R_{(i-1)}
$$

where, $R_{(i)}$, refers to the time location at the $i^{t h} \mathrm{R}$ peak. Note that, $\mathrm{i} \geq 2$ for all of the above.

As a side note, while Figure 4.2 illustrates a very short portion of the ECG signal being extracted from the example subject, Figure 4.3 depicts the result of extracting the HR values from a slightly extended version of the signal shown in Figure 4.2.

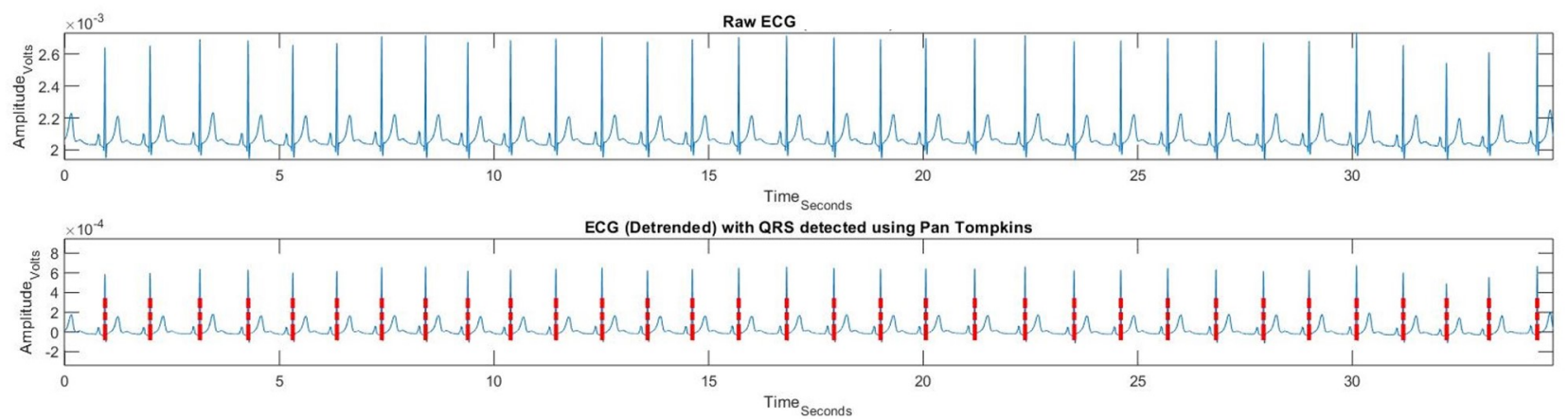

Figure 4.2: Raw ECG shown. R peaks detected using Pan Tompkins.

Nonetheless, following the calculation of HR using equation 4.1, it is of interest to point out here that, occasionally, either due to the misdetection of the R peaks by the software, or due to the origin of impulses other than from that of the pacemaker cells or, i.e., Sino-Atrial Node (SAN), located in the right atrium wall of the heart, the signals being analyzed can be interspersed with 


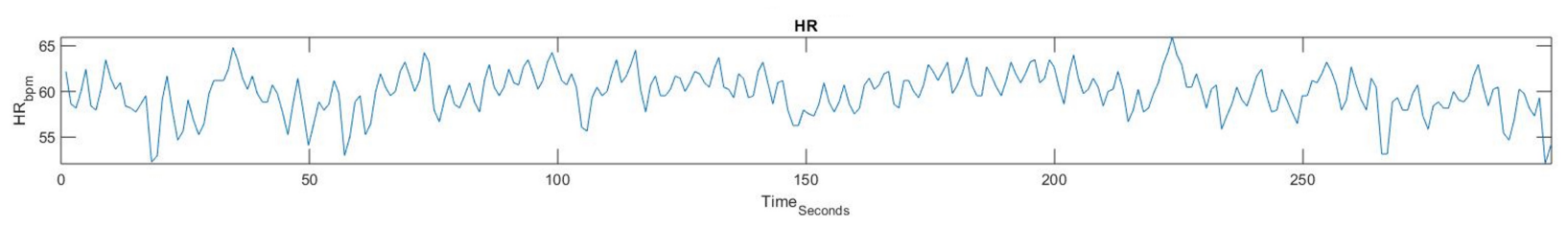

Figure 4.3: HR - corresponding to a longer ( $5 \mathrm{~min}$ ) portion of the ECG signal shown in Figure 4.2

random spikes [48]. Incidentally, the latter (impulses generated outside of the SAN) is referred to as ectopic beats [48]. Regardless, shown in Figures 4.4 and 4.5 are samples of an ECG signal and the corresponding HR signal containing ectopic beats [48]. Again, similar to the Figures 4.2 and 4.3, here as well, while the first figure, Figure 4.4, illustrates a short portion of the ECG signal that was extracted (from elsewhere) from the same example subject, the second figure, Figure 4.5, depicts the result of extracting the HR values from a slightly extended version of the signal shown in the first figure, Figure 4.4, in this case.
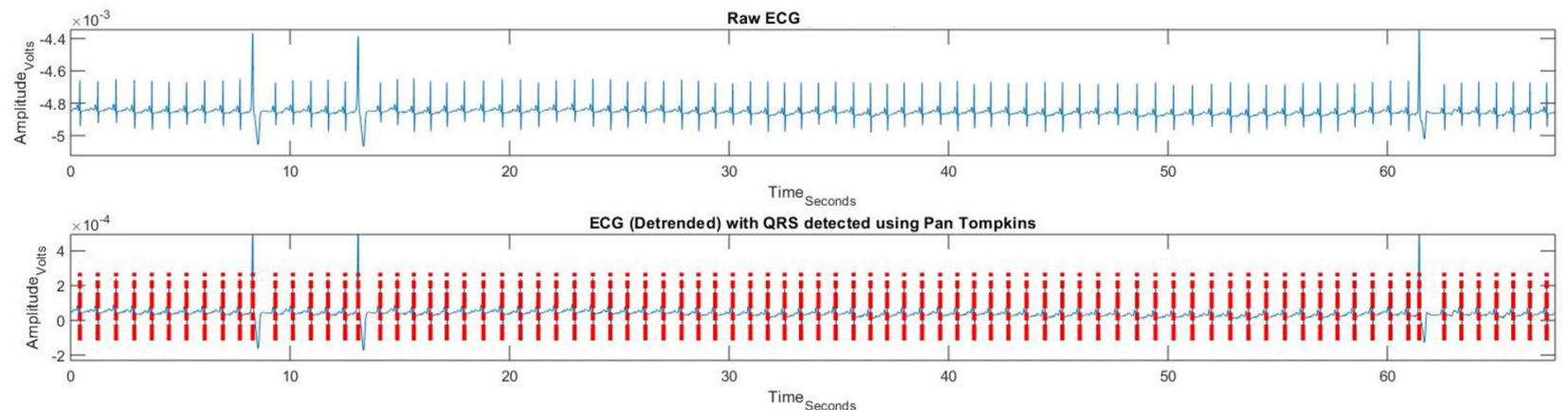

Figure 4.4: Ectopic beats picked up along with QRS resulting in occasional high HR.

All that being said, it is significant to mention here that, amid the presence of many corrective methods, such 'spikes' found in a given HR signal from the current study were removed and filled in using linear interpolation [48]. Specifically, this was done so as to comply with the standards dictated by the Task Force of the European Society of Cardiology and The North American Society 


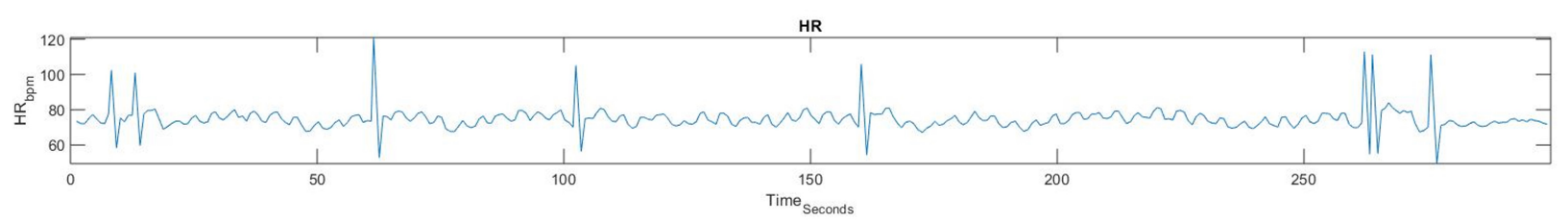

Figure 4.5: HR - corresponding to a longer $(5 \mathrm{~min})$ portion of the ECG signal shown in Figure 4.4

of Pacing and Electrophysiology, 1996 [22], mentioned earlier. In particular, the latter required, for analyses such as those pertaining to HRV, that the abnormal beats in the signal not be included [22], [49]. In fact, given that HRV was the modality through which, as discussed in the upcoming sections of the chapter, the features relating to the HR signals were intended to be captured, it was deemed necessary, whenever possible, that the abnormal beats be corrected for in the signal being analyzed.

\subsubsection{ECG Signal shortcomings}

That being said, despite being able to identify and correct the occasional ectopic or mis-detected beats happening in an ECG signal, certain types of noise in the signal were unavoidable or unfixable using simple interpolation techniques, either due to the nature of the signal, or due to the hardware used to measure the latter [48]. For example, shown in Figures 4.6 and 4.7, 4.8 and 4.9, and 4.10 respectively, are just some, not all, of the possible scenarios that one can come across when processing ECG signals.

Explicitly, an example of a circumstance in which the artifacts in an ECG signal cannot simply be fixed using linear interpolation for example, is when the signal being analyzed is affected with noise for an uninterrupted portion of the signal. Specifically, Figure 4.6 shows for example, a continuous portion of an ECG signal in which, the T waves in the signal were mis-detected as R peaks [48]. Aside, followed by the latter, shown in Figure 4.7, is the HR signal calculated using the mis-detected R peaks from the signal shown in Figure 4.6. Note that, similar to the figures from the previous section, for the second figure here, the result of the HR values is calculated using a 
slightly extended version of the ECG signal shown in the first figure. Nevertheless, it is of interest to point out here how, unlike the occasional 'spikes' caused by the ectopic or missed beats mentioned before, here, the spikes exist for continuous and prolonged periods within the full length of the signal depicted.
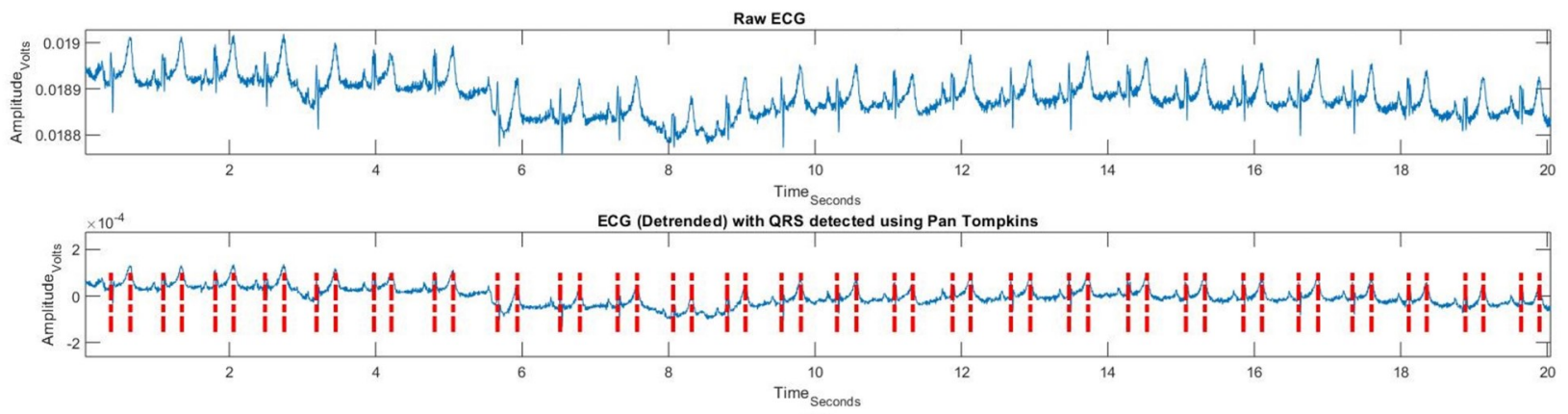

Figure 4.6: ECG - Long $T$ wave along with QRS detected as QRS using Pan Tompkins.

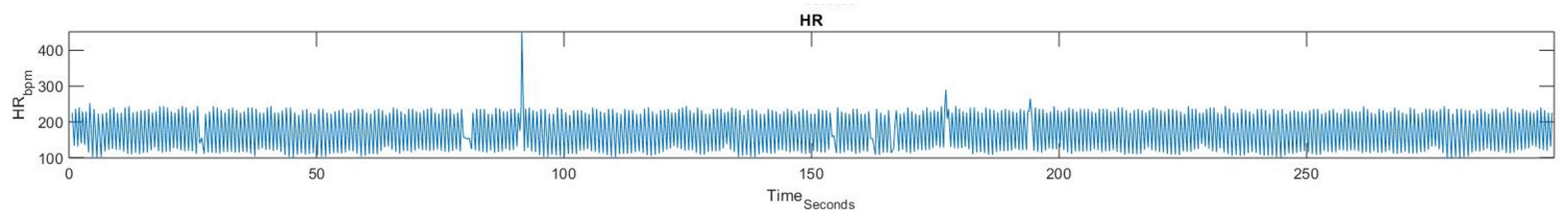

Figure 4.7: HR - corresponding to a longer ( $5 \mathrm{~min}$ ) portion of the ECG signal shown in Figure 4.6

Apart from the above, shown in Figure 4.8, are incorrectly marked R peaks, possibly detected due to movement artifacts in the ECG signal [48]. Also, shown (in Figure 4.9), is the HR signal corresponding to the latter. As a side note, similar to the figures presented thus far, the second figure here depicts the result of the HR values calculated using a slightly extended version of the ECG signal shown in the first figure. Nevertheless, like the previous example, here as well, the atypical 'spikes' occur within the HR signal for continuous and prolonged periods, i.e., almost for the entire length of the signal sampled. Especially, given that the population of interest here are individuals affected with PLMS, it is emphasized here that this is one of the cases in which, 
it is inevitable to avoid (movement-related) artifacts from the ECG signals, i.e., that are obtained through conventional means at least.
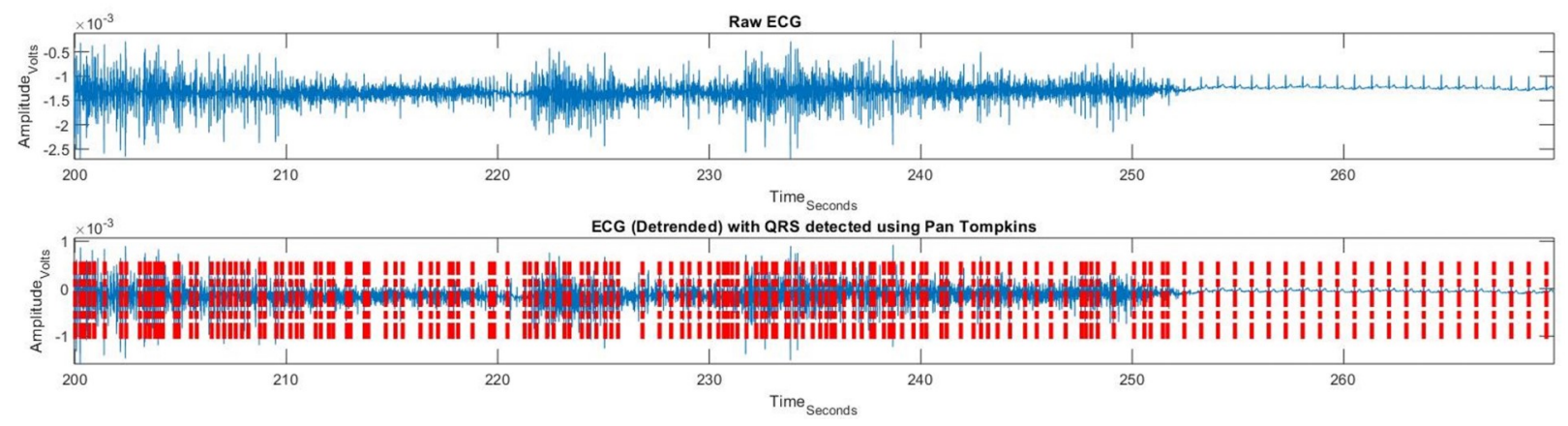

Figure 4.8: ECG - Possible movement noise erroneously picked up as QRS using Pan Tompkins.

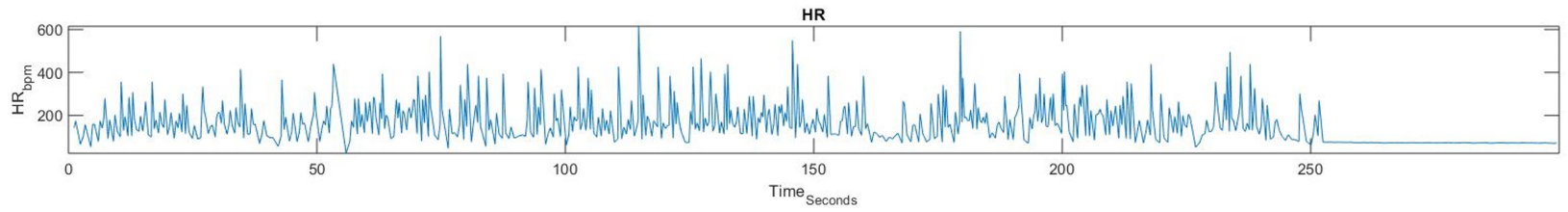

Figure 4.9: HR - corresponding to a longer ( $5 \mathrm{~min}$ ) portion of the ECG signal shown in Figure 4.8

With that said, as shown in Figure 4.10, there could also exist in the signal, other instances in which, completely irrelevant incidents can be picked up as R peaks. Specifically, given the atypical, yet symmetrical nature of the signal depicted in Figure 4.10, it is presumed that the latter could have been the result of instrumentation-related noise [48]. Nevertheless, all this brings us to the next section in which, a possible approach for dealing with, even if not solving for, such signal- or instrumentation-related issues, is discussed.

\subsubsection{Overcoming the shortcomings associated with ECG Signals}

Specifically, while for some of the artifacts mentioned earlier, the approach would be to individually recognize and fix the artifacts in the ECG signal, for others, the approach would have been 

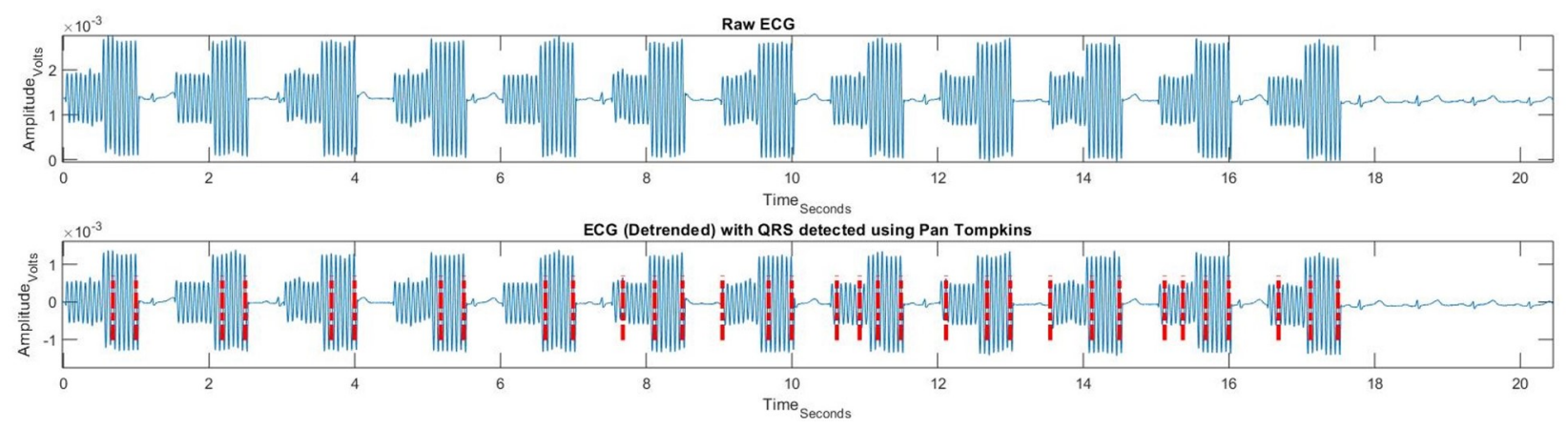

Figure 4.10: Possible instrumentation noise erroneously picked up as QRS using Pan Tompkins.

to prevent them from happening in the first place, i.e., by using, non-contact [50] electrodes for instance. Essentially, both of them would require significant modifications, either at the software, or, data acquisition level. Nevertheless, since, in the current thesis, these were out of scope, it was of interest to utilize a simple method that, at the very least, identified within the HR signal, the regions where extensive periods of erratic beats occurred. Ultimately, the goal here was not to use, as studied in the upcoming sections of the chapter, features derived from the artifact-rich regions in the HR signal to classify between PLM vs. non-PLM events. Again, as mentioned earlier, this is in line with the standards dictated by the Task Force, 1996, by [22] in which, abnormal beats were not to be included in any of the analyses conducted with regards to HRV [22], [49].

Nonetheless, shown in Figures 4.11 and 4.12, are examples of a clean and non-clean HR signal, obtained from two subjects respectively, for the length of an entire PSG session. While the 1st subplot of either of the figures represent the HR signals obtained from the entire PSG sessions of the two subjects, the 2nd subplot represents the same HR signals arbitrarily chosen to be marked with 'peak prominences' beyond $40 \mathrm{bpm}$. Aside, it is of interest to mention here that this was easily realizable using the findpeaks() function from MATLAB [51]. In essence, it was expected that, the latter would enable us to locate in the signal, the HR deviations that were not normal. That being said, in order to limit HR deviations to those that occurred for uninterrupted and extended periods of time, first, the count of the number of peaks per non-overlapping 5 min windows for the entire length of the given HR signal, was noted. Secondly, based off of an arbitrary threshold value, the counts per 5 min segment that were beyond the latter were marked. This is as shown in the 

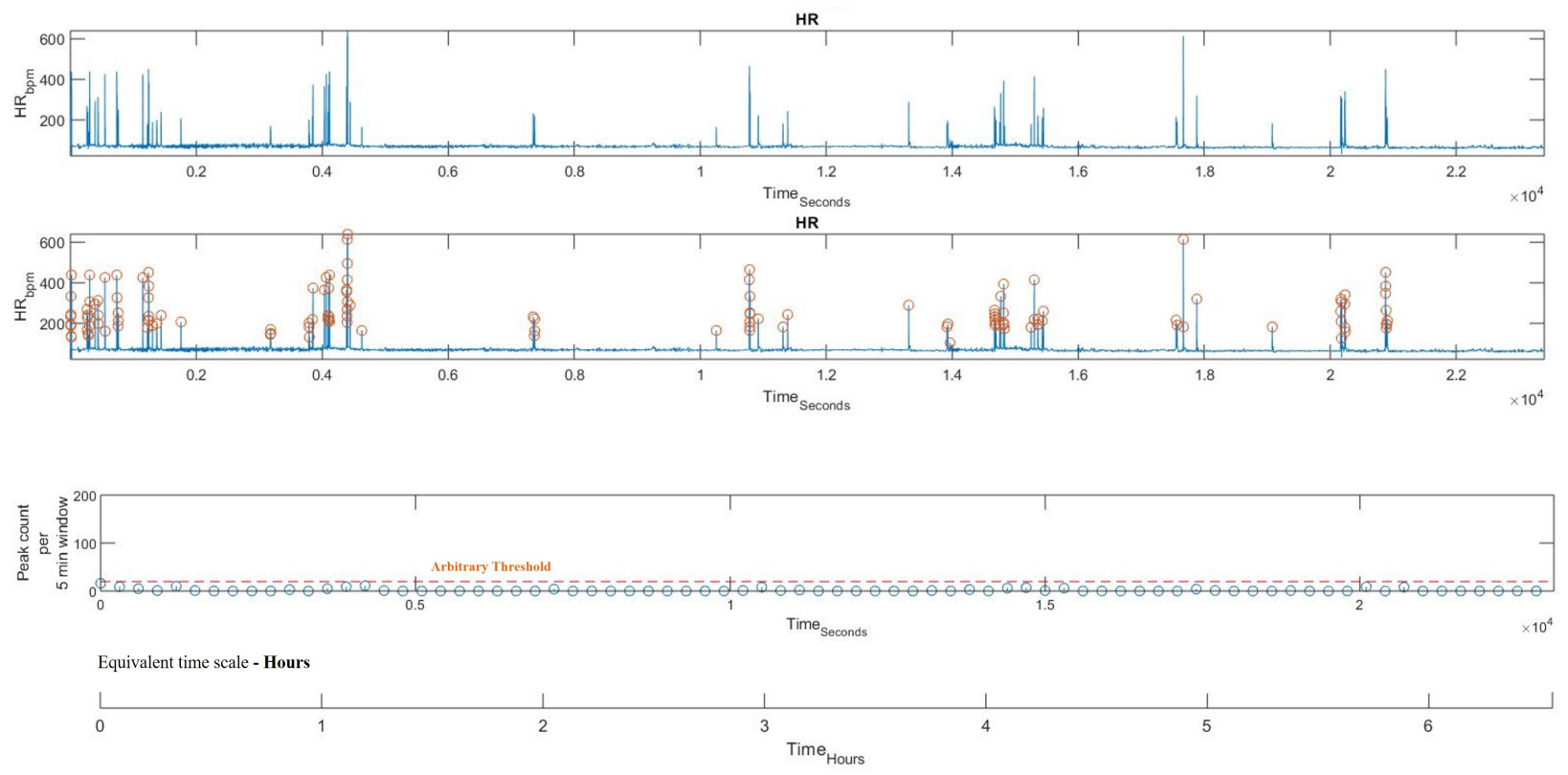

Figure 4.11: Example of a clean HR signal obtained from the entire duration of a PSG session

3rd subplots of either of the figures. In particular, note how, as required, none of the counts per windowed segment for the clean signal shown in Figure 4.11 were beyond the arbitrary threshold while, for the non-clean signal shown in Figure 4.12, certain regions in the signal happened to be beyond the threshold value. Essentially, the point of doing all of the above was to be able to mark down, in the signals being analyzed, the regions that were to be discarded from further analyses.

Lastly, it is also noted here that, given that the signals that we are dealing with belonged to that of long-term signals, i.e., signals typically lasting for 6 to 8 hours of sleep, it is highly likely that, for certain durations in the signal, that 'losses' in the signal could occur. An example of the latter is as shown in Figure 4.12 in which, for example, the non-clean HR signal shown has a small portion of the signal marked with signal loss. Similar to the regions in the signals that were marked with extended periods of sporadic beats, these regions were also deemed unusable, and hence, were also marked down to be discarded from further analyses in the sections that followed.

Once certain sections of the HR signal obtained per subject over the entire PSG session are fixed (i.e., this is applicable to the sections of the signal with occasional spikes), or discarded 

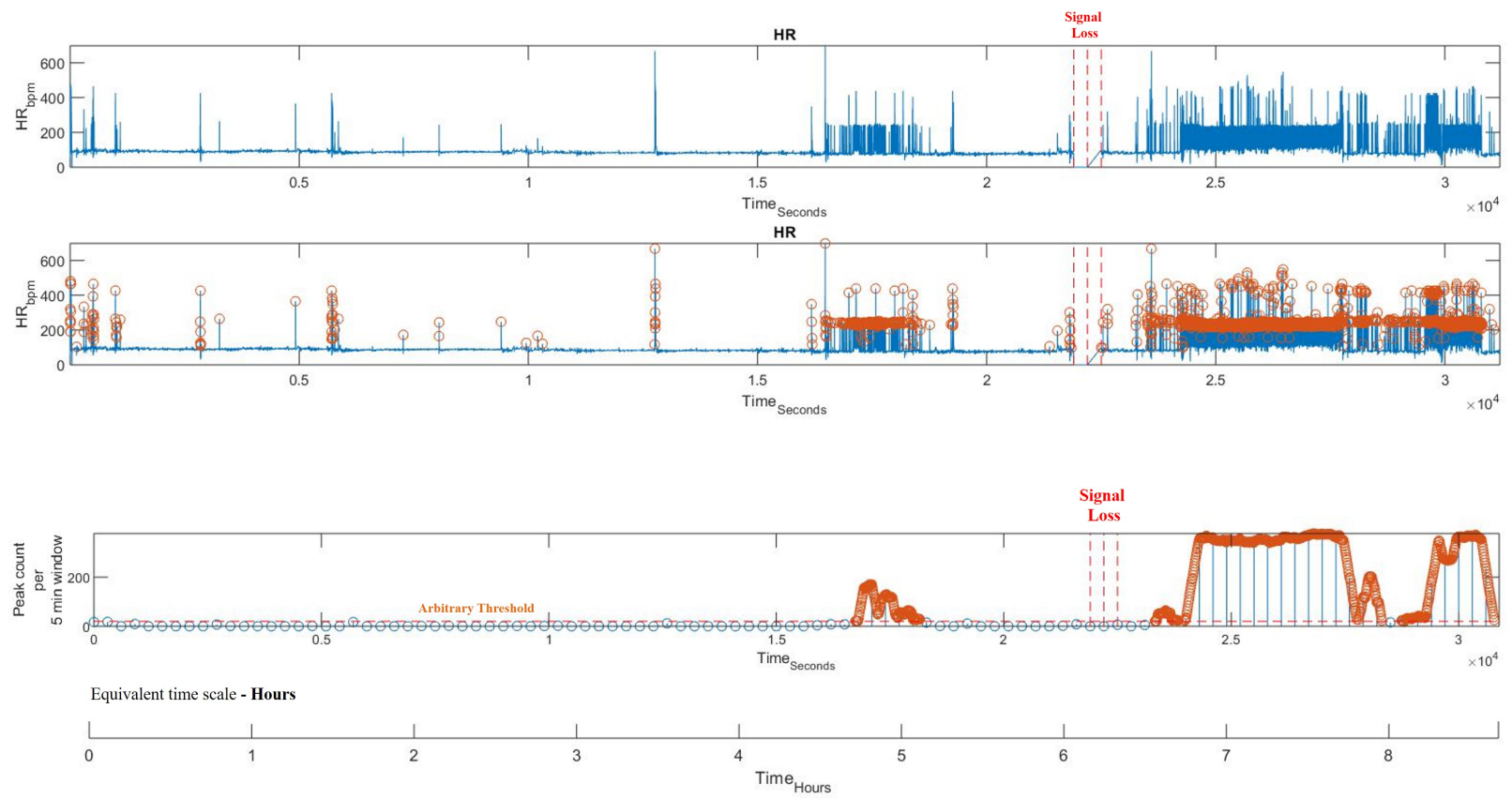

Figure 4.12: Example of a non-clean HR signal obtained from the entire duration of a PSG session

(based on signal loss or continuous periods of HR 'peaks'), the next step was to extract features from 'clean' HR signals and distinguish between the PLM and non-PLM periods of the signal based on those features.

\subsection{Feature Extraction and PLMS Classification using HR Sig- nals}

\subsubsection{Extracting core features from HR signals}

To start with, it is significant to introduce here a study conducted by [10], in which, similar to the goal in the current thesis, the authors exclusively used features based on the HR signal to discern between restful and stressful periods for a select set of stress-evoking scenarios that the participants 
in their study had to undergo, i.e., such as giving a presentation at a conference, performing surgery (applicable to surgeons), etc. [10]. To be exact, in order to classify between the different states within the various stress-inducing conditions that the subjects in their study had to undergo, the authors in [10] used features from the HR signals that were specifically related to the LF and HF components of the signal. Essentially, similar to many studies using HRV to indirectly study the behaviour of the autonomic system with respect to a given disease state (for e,g., [52]), the choice of using the frequency components of HR to distinguish between the 'stress' vs. 'rest' conditions in [10] was based on the presumption that, the latter, would be the most reflective of the autonomic nervous system [22]. Nonetheless, in the current thesis, instead of classifying between the 'rest' and 'stress' states occurring during stress-evoking scenarios, the idea here was to adopt the procedure from [10] to differentiate between 'PLM' vs. 'non-PLM' states that could occur during sleep for a given subject. Again, it is reiterated here that, as mentioned earlier, in the beginning of the chapter, the latter was conceptualized based on the assumptions reviewed in Chapter 2 with respect to the physiology of the 'flight' of 'fright' system and its relationship to PLM [15], to be exact. Moreover, the results from past studies that indicated a significant difference between the HR variables corresponding to PLM versus non-PLM events (e.g., [23] [24]), for example, added to the confidence in the feasibility of distinguishing between 'PLM' vs. 'nonPLM' states in a given subject using HR signals.

With that said, as suggested in [10], from the HR signals, the LF and HF components of the latter were first extracted. To be precise, according to step (i) dictated in [10], the HR signals being analyzed were separated into two parts, i.e., the LF (0.04 to $0.15 \mathrm{~Hz})$, and HF ( 0.15 to $0.4 \mathrm{~Hz})$ components of the signal. For example, shown below in the 2 nd subplot of Figure 4.13 is the LF and HF components of the signal obtained from bandpass filtering the HR signal shown in the 1st subplot. Note that, for illustration purposes, only a specific segment from the signal from the entire PSG session of the chosen subject is shown. Moreover, it is also of interest to mention here that, in order to center the signal around zero, the detrended form of the signal was obtained before applying the band pass filter.

Nonetheless, moving on to step (ii) of [10]'s methodology, the latter required that for each, 

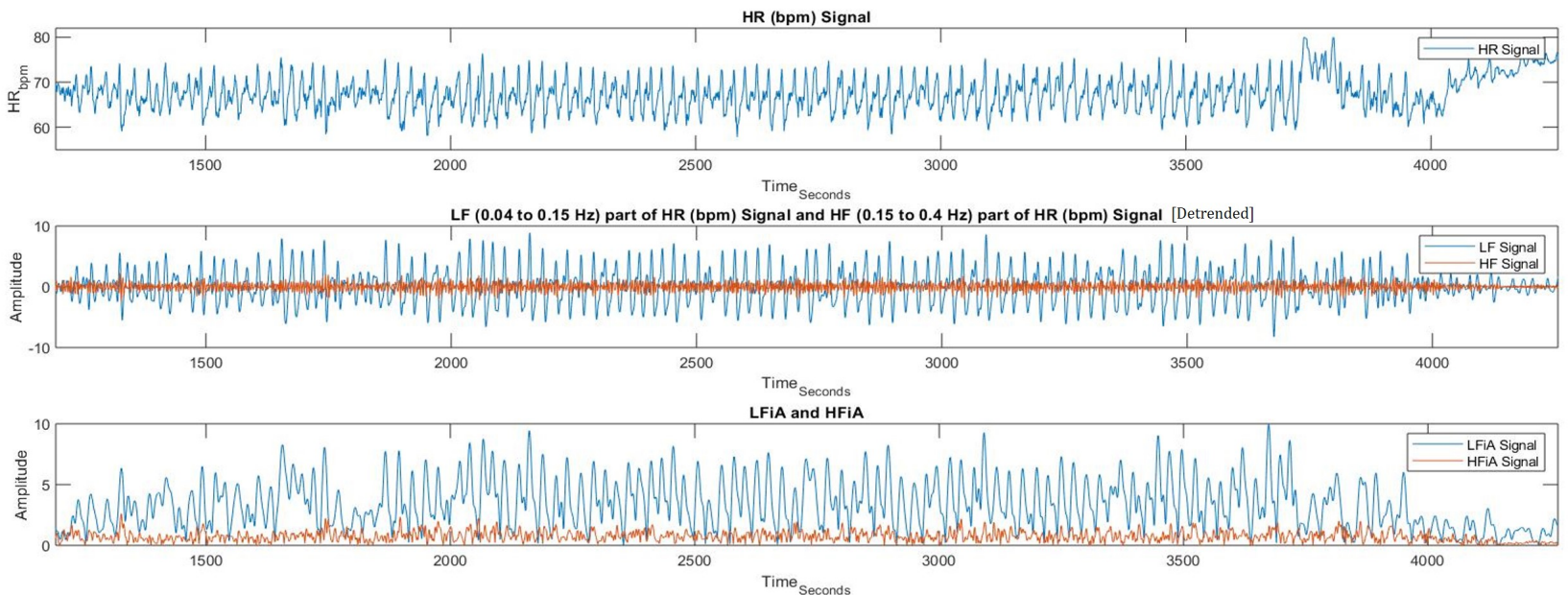

Figure 4.13: Implementation of HT on a sample HR signal

i.e., LF and HF parts of the signal obtained per subject, Hilbert Transform (HT) be applied to the signals. In short, the latter is a technique that produces the analytical counterparts of the LF and HF components of the signal in this case, from which, the instantaneous amplitudes (for each of those signals), can be obtained, at any given instant [10]. Referring to Figure 4.13, from each of the analytical signals, i.e., one for the LF, and another for the HF component of the signal, the instantaneous amplitude, i.e., referred to as LFiA and HFiA, respectively by [10], at every point in time, is as shown in the 3rd subplot of Figure 4.13. In particular, the latter illustrates, for the LF and HF parts of the signal shown in the 2nd subplot, the corresponding instantaneous amplitudes, i.e., the LFiA and HFiA at every point in time.

Finally, for the last step, i.e., step (iii), the authors in [10] applied 5 min windows across the LFiA and HFiA signals obtained in their study, sliding by 10s, and took the mean per window with outliers excluded. As a side note, it is of interest to mention here that, in the current analysis, instead of taking mean, median was used to forego the step of exclusively looking for outliers at this stage in the analysis. Moreover, assuming that the slightly or heavily 'spiked' regions in the HR signals from before were either corrected for (using linear interpolation), or discarded from consideration (using the developed peak indicator), respectively, it is highly unlikely that we would come across unruly deviations in the LFiA and HFiA signals analyzed here. Regardless, the median 
values per windowed segment were used when implementing the current step. Specifically, this is represented in the 2nd subplot of Figure 4.14, in which, for the LFiA and HFiA signals illustrated in the 1st subplot of Figure 4.14, the former demonstrates the simultaneous result of taking the median per 5 min window, i.e., every 10s, across the signals. Note that, unlike Figure 4.13 though, in which only a portion of the resultant signals were shown, here, the resultant signals obtained from the entire PSG session for the chosen subject, is shown.

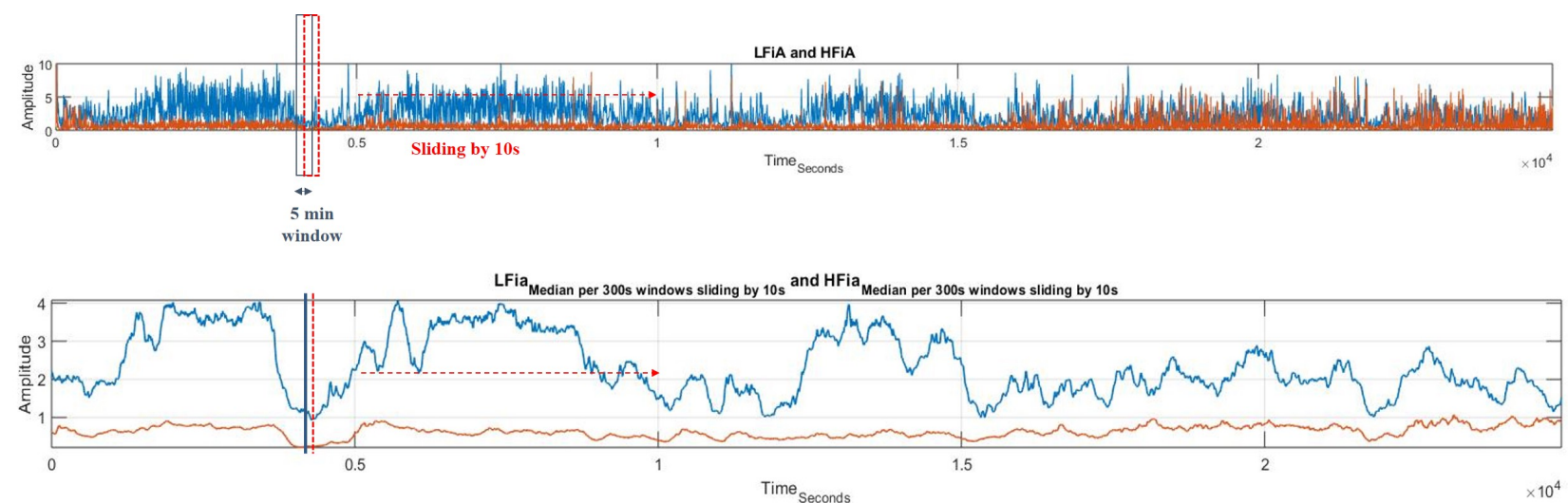

Equivalent time scale - Hours

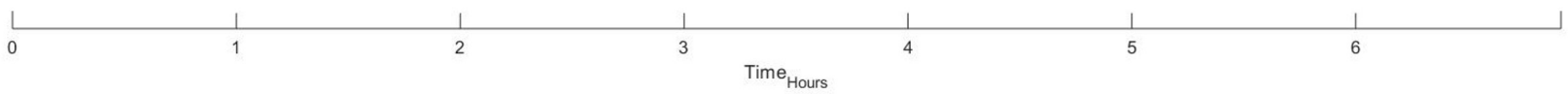

Figure 4.14: The core features, i.e., $L F i a_{\text {median }}$ and $H F i a_{\text {median }}$, extracted from a sample HR signal, obtained from the entire duration of a PSG session

\subsubsection{Individualized 'PLM and Sleep Labelling' of data}

Following the extraction of the HR-based features deemed most relevant for PLM classification, i.e., median LFiA and HFiA respectively, it was of interest here to label each occurrence of these features with a PLM or non-PLM label, respectively. In order to do so, as mentioned in the beginning of the chapter, the PLM segmentation information obtained per subject from Chapter 3 was used. However, it is of interest to note here that instead of having just two labels, i.e., those belonging to 'PLM' and 'non-PLM', or in other words, 'abnormal' and 'normal', three main la- 
bels were used here instead, namely, 'abnormal', 'normal - no movements', and 'normal - some movements'. Specifically, the separation for the labels for normality was done so as to differentiate between the normal regions in the signal in which, no movements of any kind occurred, versus others, in which, non-PLM movements occurred. The idea here, was to keep separate, the HR changes that happened due to non-PLM movements [53], and those that happened exclusively due to PLM.

Aside from the above, it is of interest to mention here that, the same window size and sliding frame that was used earlier for extracting the HR-based features was used here as well to determine, using the segmented PLM signal, the respective 'normal' or 'abnormal' labels for each row of data corresponding to the two feature values. This is as shown in Figure 4.15 which, is organized in a bottom-up fashion. Nevertheless, as shown in the figure, it is also of interest to point out here that, just like the distinction in the labels for normality, the 'abnormal' label was further subdivided into the ranges ' $1 \leq \mathrm{a}<25 \%$ ', ' $25 \leq \mathrm{a}<50 \%$ ', '50 $\leq \mathrm{a}<75 \%$ ', ' $75 \leq \mathrm{a}<100 \%$ ', and 'a $=100 \%$ ' where, a stands for the abnormality level. To elaborate, the latter represented the proportion of the amount of time per 5 minute window that corresponded to PLM. In other words, presuming that the period between the start and end of a PLM series (as described in Chapter 3 to be made up of at least 4 CLM long [7]) was considered to be 'abnormal', the appropriate 'abnormal - percent' range was determined based on the portion of time per 5 min window that corresponded to the 'abnormal' regions marked per subject. Note that, this meant that, even the IMI between the abnormal LM in the PLM series was included in the calculation for the level of abnormality. That being said, it is also of interest to mention here that, the whole point of subdividing the label for abnormality was to be able to distinguish between, for example, a 5 min windowed segment that corresponded to an 'abnormal' PLM region for the full 5 min versus others, in which, say, only a small portion of the 5 min windowed segment corresponded to the marked 'abnormal' PLM region. In particular, the purpose of the latter was to be able to accommodate the differences in HR changes that could happen due to the differences in the amount of time per windowed segment that corresponded to PLM events. The latter was especially deemed significant given that only a single median value per feature was used in order to represent the HR variation per windowed segment. Nonetheless, 
the logic-based implementation of the entire labelling process, i.e., including the assignment of the 2 normal and 5 abnormal labels overall, is as shown in Figure 4.15.

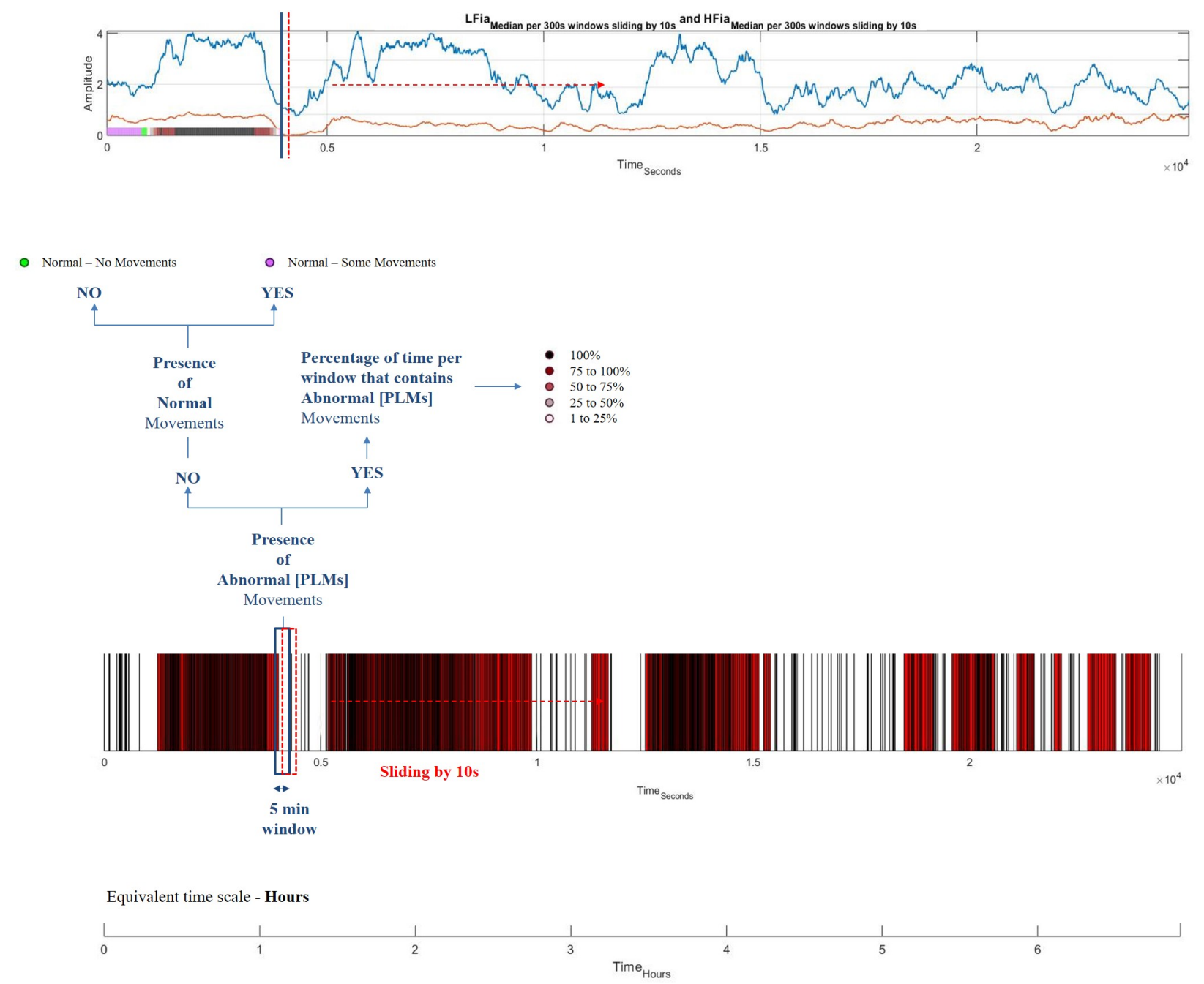

Figure 4.15: The application of normal and abnormal (PLM) labels across the core features extracted from a sample HR signal, obtained from the entire duration of a PSG session

With all that said, aside from labeling the features based on PLM, it was also of interest here not to include data points that did not correspond to sleep. The basis for the latter was so as to mimic the clinician's PLM scoring [7] in which, the PLM corresponding to awake periods was not considered. However, so as not to be too restrictive, instead of only considering values that corresponded to $100 \%$ sleep, it was arbitrarily chosen that the values corresponding to 75 to $100 \%$ 
sleep be included in the analysis going forward. That being said, similar to the PLM labelling discussed above, the percent of sleep per window was based on the amount of time per 5 min window that corresponded to the time spent sleeping by the subject under question. This is as illustrated in Figure 4.16 in which, like in Figure 4.15, the steps involved are organized in a bottom-up fashion.
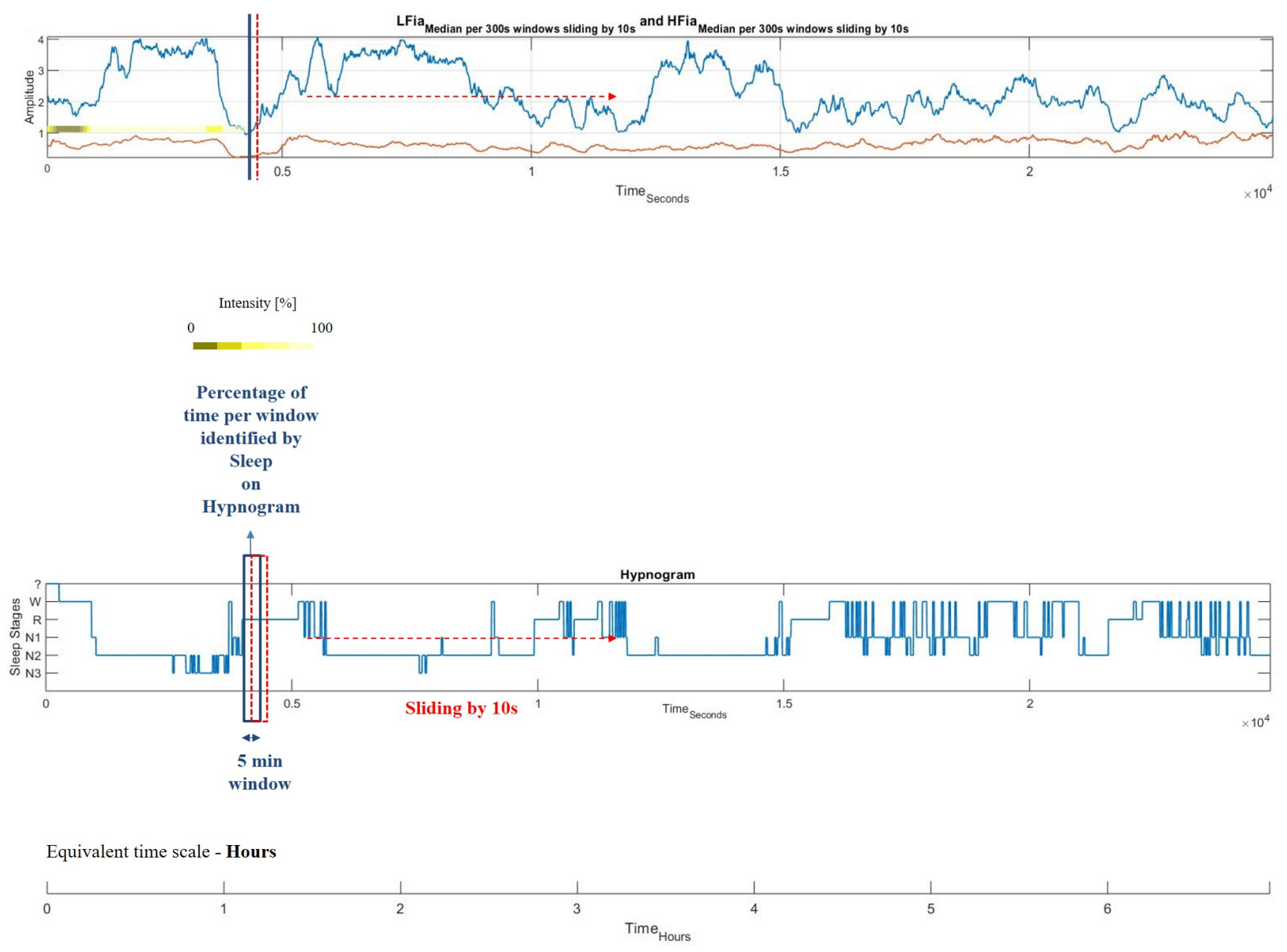

Figure 4.16: The application of sleep weightage across the core features extracted from a sample HR signal, obtained from the entire duration of a PSG session

Apart from all of the above, it is of interest to mention that, hereafter, for each of the Datasets A and B, the results obtained are separated into 2 groups. Specifically, they were subdivided into two groups such that, while one group comprised of individuals that had PLM greater than or equal to 
15 per hour, the other consisted of individuals who had PLM less than 15. The number 15 was chosen based off of The International Classification of Sleep disorders - Third Edition (ICD-3) [1] in which, an individual was designated as being abnormal if they had a PLM greater than or equal to 15 per hour.

Along the same lines, shown in Figures 4.17 and 4.18 are sample subjects belonging to either of the groups mentioned above. Specifically, while Figure 4.17 illustrates signals obtained for an individual with PLM greater than or equal to 15, Figure 4.18 represents the same for an individual with PLM less than 15. Note that, longitudinally, for either of the individuals, the segmented EMG, hypnogram, 'Peak' indicator, median LFiA and HFiA, etc., discussed thus far, are illustrated in the 1st, 3rd, 4th and 2nd subplots respectively.

Last, but not least, also shown near the topmost parts of these figures are a series of 2D scatter plots of the values of the median LFiA and HFiA features plotted against each other for each subject, respectively. In particular, these are shown for the three case scenarios, namely, 1, 2, and 3. In the current analysis, while scenario 1 included all of the data per subject corresponding to the 2 'normal' and 5 'abnormal' sub-designations that were defined before, scenarios 2 and 3 only included the data per subject corresponding to the 'normal-no movement' vs. all of the 5 'abnormal' sub-designations, or 'normal-no movement' vs. the 'abnormal - 75 to 100 (inclusive of the 100\%', respectively. Specifically, it is noted here that, while for case scenarios 1 and 2 , the data pertaining to all the 5 'abnormal' sub-designations were combined to represent one 'abnormal' class per subject, for scenario 3, only the data corresponding to the '75 to 100 (inclusive of the 100\%)' range was used in order to represent the 'abnormal' class for each subject. On the other hand, the data pertaining to the 2 'normal' sub-designations for each subject were kept separate from each other across all case scenarios. As a side note, for the sake of simplifying the terminologies, from here onwards, the letters ' $\mathrm{N}$ ', 'S', and 'A', were used for the terms 'normal-no movements', 'normal-some movements', and 'abnormal'. Moreover, for the 'A' class, a hyphen and the respective percent range was used in order to denote the portion of time per segment that corresponded to a PLM region, as defined earlier. Nevertheless, in order to be absolutely precise with regards to the data encompassed within each of the case scenarios being analyzed per subject, while scenario 1 

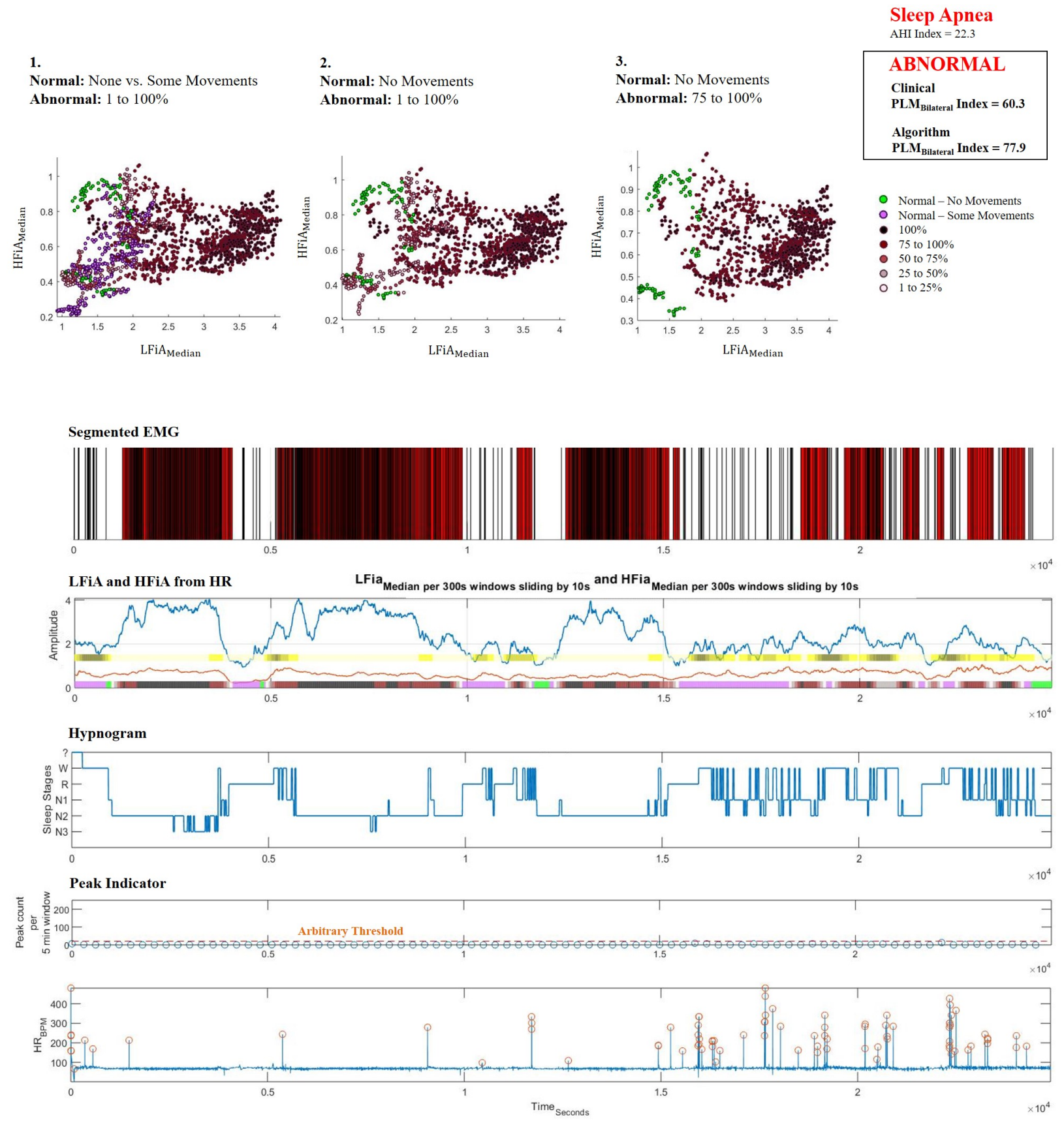

Equivalent time scale - Hours

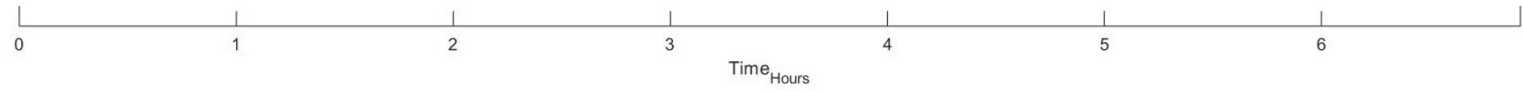

Sleep Intensity [\%]

$0 \quad 100$

Figure 4.17: Sample Abnormal Subject (PLM $\geq 15)$ 
1.

Normal: None vs. Some Movements Abnormal: 1 to $100 \%$
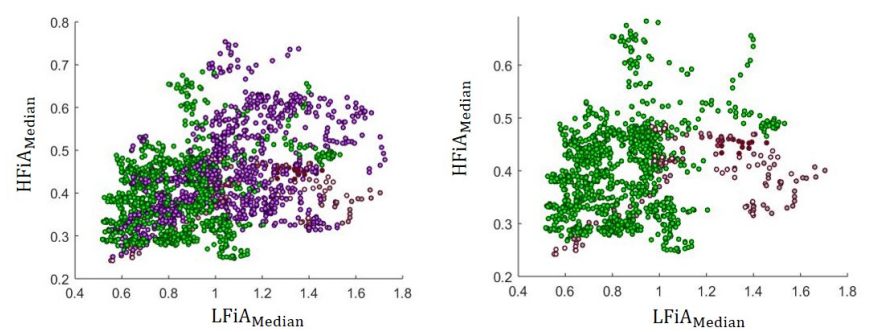

Normal: No Movements

Abnormal: 1 to $100 \%$

Segmented EMG

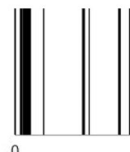

3.

Normal: No Movements

Abnormal: 75 to $100 \%$
Sleep Apnea AHI Index $=3.2$

\section{NORMAL}

Clinical

$\mathbf{P L M}_{\text {Bilateral }}$ Index $=0$

Algorithm

$\mathrm{PLM}_{\text {Bilateral }}$ Index $=2.2$

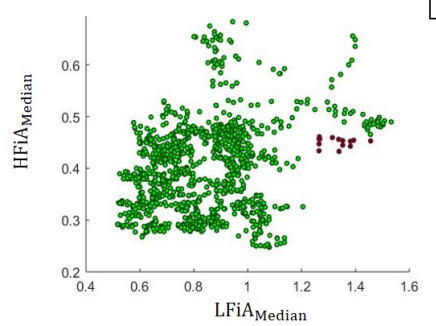

Normal- No Movements

Normal - Some Movements

- $100 \%$

- 75 to $100 \%$

- 50 to $75 \%$

25 to $50 \%$

1 to $25 \%$
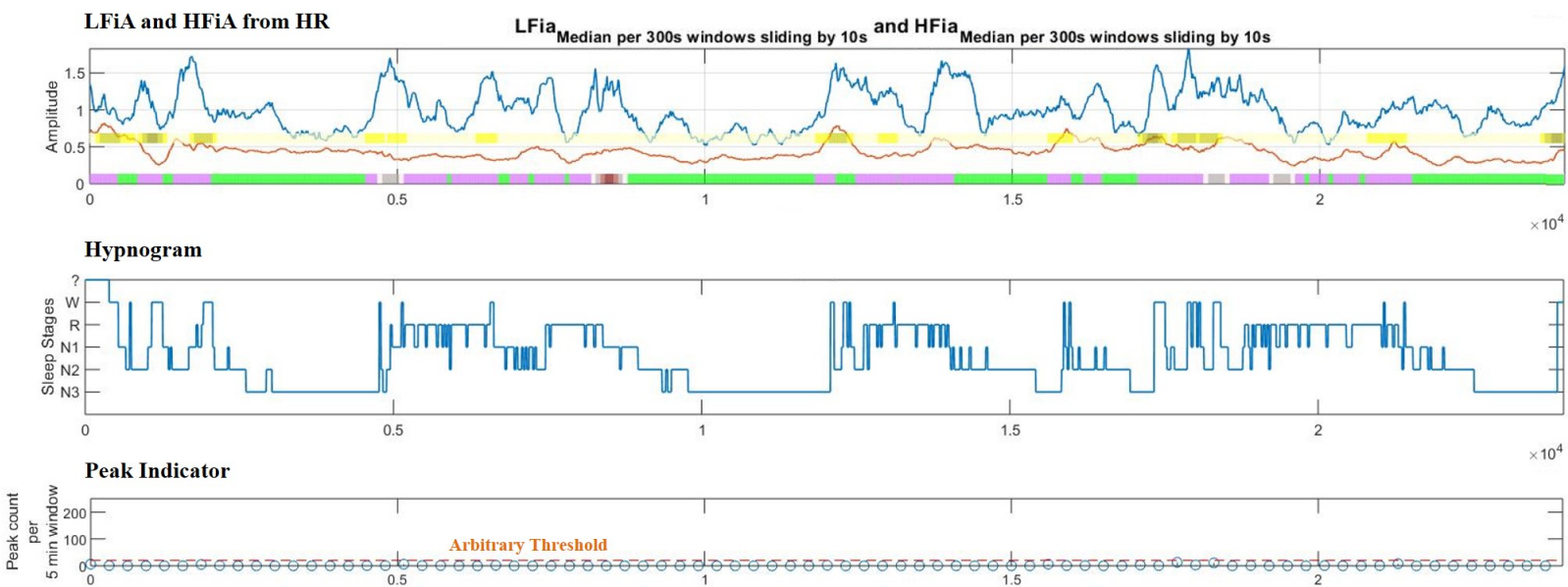
$\times 10^{4}$

Arbitrary Threshold

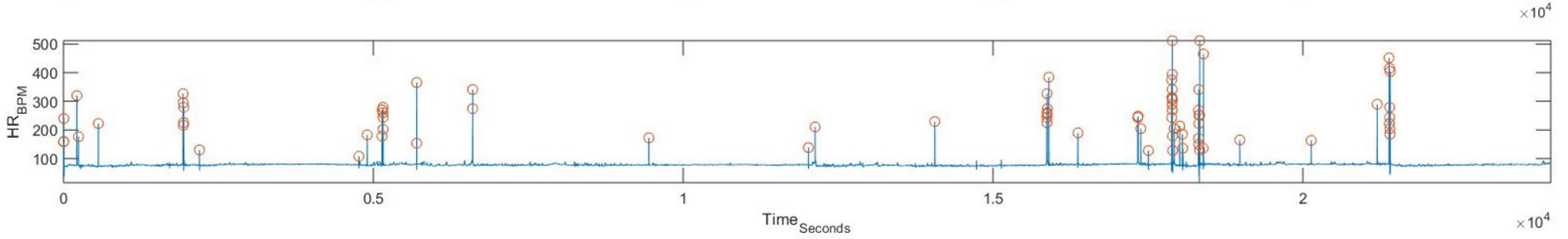

Equivalent time scale - Hours

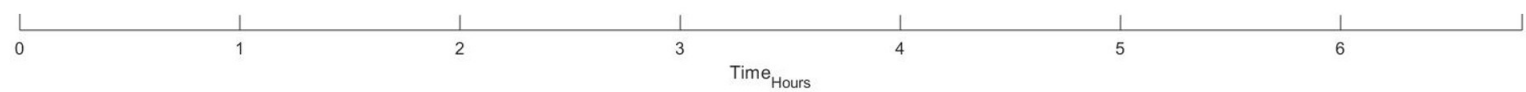

Sleep Intensity [\%]

$0 \quad 100$

Figure 4.18: Sample Normal Subject $(\mathrm{PLM}<15)$ 
comprised of data from the three classes, i.e., that of ' $N$ ', 'S', and 'A - 1 to 100\%', the other 2 case scenarios comprised of data from 2 classes each, i.e., either ' $N$ ' and 'A - 1 to 100\%' or, 'N' and 'A - 75 to 100\%', respectively. The purpose of analyzing 3 scenarios, after all, was to be able to differentiate, with respect to the extremely normal (' $\mathrm{N}$ ') condition, the HR changes that occurred due to the exclusively abnormal ('A - 1 to $100 \%$ ') or, extremely abnormal ('A - 75 to $100 \%$ (inclusive of the 100\%') condition, respectively. In essence, the idea here was to be able to remove any interference or changes in the HR that could happen due to non-PLM movements [23], [53], and, also, be able to affirm whether there is a difference in the intensiveness in the changes in HR depending on whether a given HR data point extracted from a subject corresponded to a full 5 minutes of PLM occurrence (100\%) versus only, say, 2.5 out of the 5 minutes corresponding to PLM occurrence $(50 \%)$.

Aside, note that, the idea for the series of 2D scatter plots per subject was grasped from [10], to be able to visualize, per subject, the presence of, if any, distinct patterns in the arrangement of the data from the two select features based on their 'normal' or 'abnormal' designations. That being said, this brings us to the next section in which, the choice of classifier and performance metrics used, in relation to the analyses described above, are discussed.

\subsubsection{Classifier and Performance metrics}

Despite overcoming the challenges imposed by 'ectopic' beats for example, note that, imbalanced data, i.e., having more data belonging to one class (majority class) versus another (minority class) [54], was yet another issue that was commonly encountered in the current analysis. Particularly, for each of the individual being studied here, the proportion of data belonging to the 'abnormal' vs. 'normal' classes varied from one individual to another. In other words, not all the individuals had equal amounts of data belonging to all the classes at all times. For example, this can be deciphered from the Figures 4.17 and 4.18 shown earlier, i.e., from the scatter plots of the figures, in particular. Specifically, note how, for the subject being analyzed in Figure 4.17, there is a higher proportion of data belonging to the 'abnormal' class across all scenarios, whereas, for the subject being analyzed 
in Figure 4.18, the proportion of data belonging to the 'normal' class was rather high compared to that of the 'abnormal' class across all the scenarios. In essence, if left unaltered, as summarized in [54], it is highly likely that the data belonging to the minority class, due to its small sample size [55], or overlapping nature [56] for example, could end up being predicted as belonging to the majority class. Keeping this in mind, going forward, the classifier used, the modifications made to it, etc., is as elaborated below.

To start with, ensemble learning methods, i.e., specifically bagging, was chosen to be used in the current study. Principally, 'ensemble' methods involve the prediction of a new instance by learning from a group of classifiers instead of one [57]. Essentially, by doing so, not only does the latter increase the overall accuracy relative to if only a single classifier were used, but by specifically using bagging ensemble techniques, it was hoped that the class imbalance problem, mentioned earlier, would be addressed [54]. In essence, bagging involves training the respective model, on each iteration, on a randomly chosen group of samples from a given dataset [57]. By doing so, it is expected that the class imbalance problem is addressed from a data 'pre-processing' perspective [54]. Note that, in the current study, the bagged ensemble made up of decision trees was used. Moreover, it is also noted here that the MATLAB Classification Toolbox was used in order to execute the latter [58]. Aside, it is of interest to mention here that, the k-fold 5 crossvalidation method was used. In essence, the latter involves dividing up the data being studied into 5 equal portions, i.e., training the chosen classifier on 4 portions of the data, and testing the latter on the remaining 1 portion of the data for a total of 5 iterations [59]. This means that, each data point in the set being studied gets a chance to be in the testing portion at least once. Nonetheless, note that, by executing the training and testing process for a total of 5 iterations, the latter results in being able to test the performance of the model being studied for a total of 5 times. Hence, to evaluate the metrics chosen to evaluate the classification model (discussed in the upcoming section), the average of the 5 iterations is used [59]. Moreover, the reader is reminded here that, as mentioned at the beginning of the chapter, the classifier is trained and tested independently on each subject, to be able to discriminate between the periods with 'PLM' vs. 'no PLM', or, to be exact, the 'abnormal', 'normal-some movements', and 'normal-no movements' regions of that subject. 
That being said, shown in Figure 4.19 is the result of applying the 5-fold cross-validated ensemble classifier, implemented using [58], on the subject represented on Figure 4.17 from before, across all the 3 scenarios. Specifically, shown in the figure is the class boundaries obtained from applying the mentioned classifier against the known labels of the data pertaining to the features for each case scenario of that subject. To be precise, as implied by the labels (for the regions) in the figure, if a new data point was further sampled from the subject being analyzed, it would be predicted as belonging to the respective region encompassed by the boundaries defined in the figure.

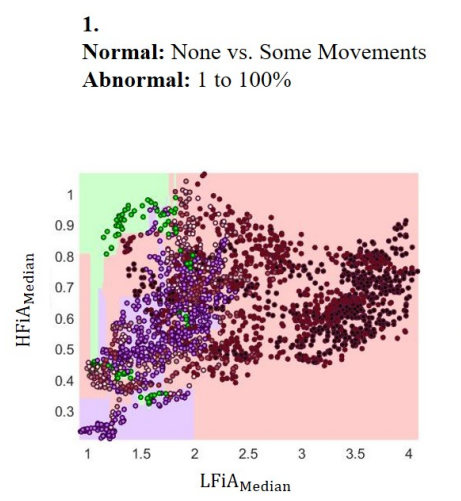

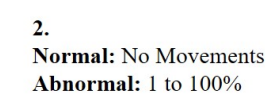

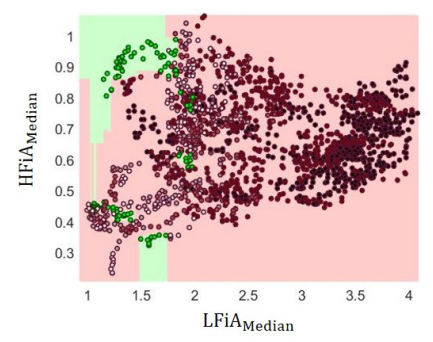

Normal (No Movements) Region
Normal: No Movements

Abnormal: 1 to $100 \%$

Normal (Some Movements) Region
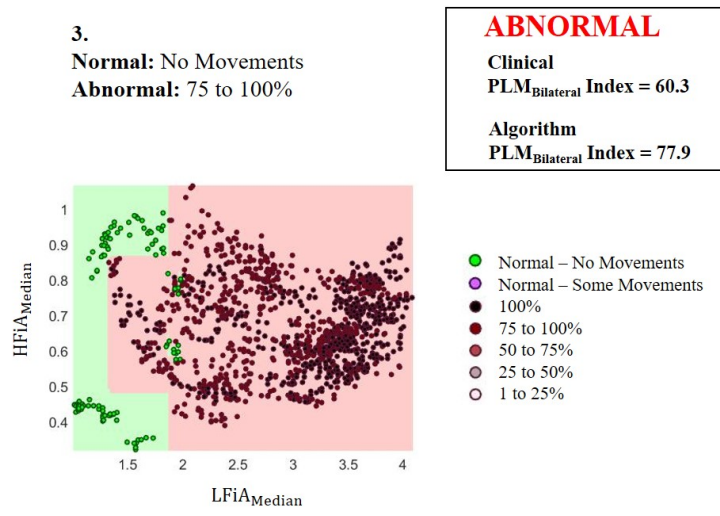

Figure 4.19: Implementation of bagged tree classifier on an Abnormal Subject

In order to evaluate the performance of the classification per subject, the parameters, True Positive Rate, $T P_{\text {rate }}$, True Negative Rate, $T N_{\text {rate }}$, False Positive Rate, $F P_{\text {rate }}$, and False Negative Rate. $F N_{\text {rate }}$, were calculated. Specifically, for binary classification for example, each can be calculated using the following [54]:

$$
\begin{aligned}
& T P_{\text {rate }}=\frac{T P}{T P+F N} \\
& T N_{\text {rate }}=\frac{T N}{F P+T N}
\end{aligned}
$$




$$
\begin{aligned}
& F P_{\text {rate }}=\frac{F P}{F P+T N} \\
& F N_{\text {rate }}=\frac{F N}{T P+F N}
\end{aligned}
$$

where, TP referred to True Positive, TN referred to True Negative, FP referred to False Positive, and FN referred to False Negative [54]. To elaborate, while TP and TN referred to the data that belonged to the positive and negative class respectively, that were correctly classified as belonging to their respective classes, FP and FN referred to the data that belonged to the negative class and positive class respectively, that were incorrectly classified as belonging to the opposite classes. Since, in the current analysis, we were interested in identifying the group of data per subject that corresponded to PLM regions, from here onwards, any reference to the positive class represented the group of data per subject that corresponded to the PLM regions, and negative class represented the group of data per subject that corresponded to the regions without PLM. This meant that, from here onwards, any of the 5 abnormal and, 2 normal sub-designations, defined earlier, were interchangeably referred to as belonging to the positive and, negative class, respectively.

Aside from the above, it is of interest to introduce here a single measured score, i.e., the Matthews Correlation Coefficient (MCC) [60], that can be calculated using the values of TP, TN, FP, and FN, mentioned above [61]. Essentially, other than providing a single measure, the MCC score has been shown to be a suitable performance metric that can be used for imbalanced data [61], as is the case here. Note that, although this measure is typically used to evaluate the performance of binary classification, it has also been modified [62] and been used in the multi-class case [62], [63]. In fact, it is acknowledged here that the MCC scores presented in the current thesis were evaluated using the software developed by [62], available online [64].

That being said, it is also of interest to emphasize here the choice of the above parameters, over other commonly used performance metrics such as Classification Accuracy (CA), for example. Again, given that we are dealing with imbalanced data here, it was crucial that the performance 
metrics such as CA was not used here [54]. Specifically, unlike the above mentioned metrics, given that CA only calculated the total number of correctly classified instances, it is highly likely that the proportion of data belonging to the minority class, that is incorrectly classified, would go unnoticed [54].

With that said, the results of the analyses conducted across all three scenarios, for the subjects from Datasets $\mathrm{A}$ and $\mathrm{B}$, is as shown in the subsequent section. Before proceeding to the results though, it is significant to mention here that, the subjects from both Datasets A and B did not include individuals with major rhythm-related heart defects. For future work, provided that the regions in the signal in which the rhythm-related problems occurred were identified, it is of interest to reciprocate the analyses conducted here on those subsets of individuals, and observe the similarities and differences in their results with that of those obtained here.

\subsubsection{Classification results on Normal (PLM Index $<15$ ) and Abnormal groups (PLM Index $\geq 15$ ) across the 3 scenarios}

Note that, except for the two subjects that were identified as outliers from Dataset B (in Chapter 3) from before, and a few subjects from Datasets A and B, all the other subjects from both the datasets were included in the upcoming analyses (for case scenarios 1 and 2, at least). Specifically, except for the outlier subjects, the reason for not being able to include the few subjects from each of the datasets was because they had no data points from some of the classes, i.e., the 'A', 'N' or 'S' classes. For example, it cannot always be expected that, a subject with a PLM Index score of 0 , for instance, would end up having data from the 'A' region, and so forth. Nevertheless, presuming that binary or multi-level supervised classification methods (that was used here) requires data from all of the classes, it is anticipated that, for future work, other means, such as those of single-class classification [65], for example, could be delved into for analyzing such kinds of subjects. That being said, for other cases, the issue could be addressed by obtaining more data from the subjects of interest across multiple sessions for example, through easily attainable means such as wearables for instance [9], but since this is beyond the scope of the current study, this is left, at this point in 
time, as a suggestion that could perhaps be looked into for future work.

Moreover, it is of interest to mention here that, for some of the subjects used in the study, i.e., particularly those belonging to the normal groups (PLM $<15$ ), case scenario 3 was not applicable. For example, say a subject contained values from the extremely normal ('N') and exclusively abnormal ('A-1 to 100\%') classes for example, it may be the case that none of the HR data from this subject corresponded to a PLM region for more than $75 \%$ of their segmented lengths, i.e., which corresponds to data from the extremely abnormal class ('A-75 to 100\%'). In that case then, while case scenario 2 (that classifies between the extremely normal and exclusively abnormal classes) can be analyzed for this subject, case scenario 3 (which requires the HR data from the subject being analyzed to correspond to PLM regions for more than $75 \%$ of their segmented lengths) would not be applicable since, there is no data from the extremely abnormal ('A-75 to 100\%') class to be able to train the classifier. Nevertheless, since case scenario 3 is just an extension of the current analyses to be able to explore, from applicable subjects, the distinct feature spaces that the HR data from the extreme normal and extreme abnormal regions (per subject) occupy, the latter is not considered to be an issue here. All that being said, it is of interest to indicate here that, the $\mathrm{N}$ value, i.e., of the number of subjects being analyzed (who had the respective classes pertaining to each of the case scenarios being analyzed) is depicted in the figures summarizing the results from this section, i.e., in Figures 4.20 and 4.21.

Along the same lines, it is of interest to mention here that the results from the $T P_{\text {rate }}, T N_{\text {rate }}$, $F P_{\text {rate }}$, and $F N_{\text {rate }}$, of the $\mathrm{N}$ number of subjects from each case scenario is summarized in, what's referred to as, confusion matrices [54]. Specifically, these are shown in the first and third rows of Figures 4.20 and 4.21. To be exact, within each confusion matrix depicted per case scenario, the values obtained from taking the median of the scores for $\mathrm{N}$ number of subjects per case scenario is shown. Moreover, shown below each confusion matrix is also a boxplot containing the spread of the scores of $\mathrm{N}$ subjects.

That being said, note how, for either of the datasets, the results are categorized into the normal (PLM < 15) and abnormal (PLM $\geq 15)$ groups [1], as mentioned earlier. Nevertheless, before looking into the differences or similarities in the scores between the normal and abnormal groups, 
Normal: None vs. Some Movements

Abnormal: 1 to $100 \%$

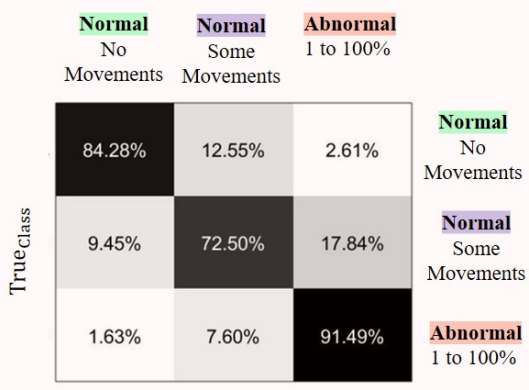

Predicted $_{\text {Class }}$
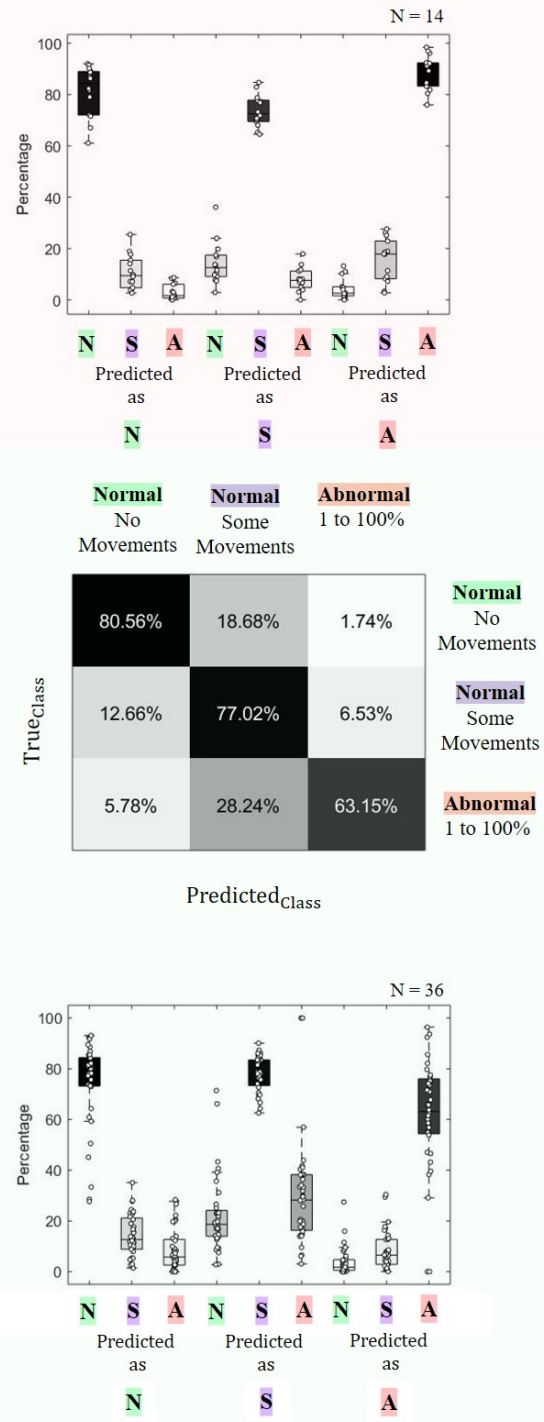

Normal: No Movements

Abnormal: 1 to $100 \%$

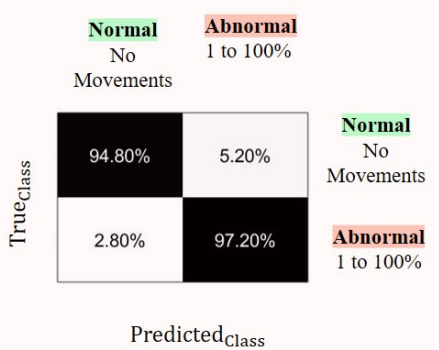

ABNORMAL Group

PLM Index $>=15$
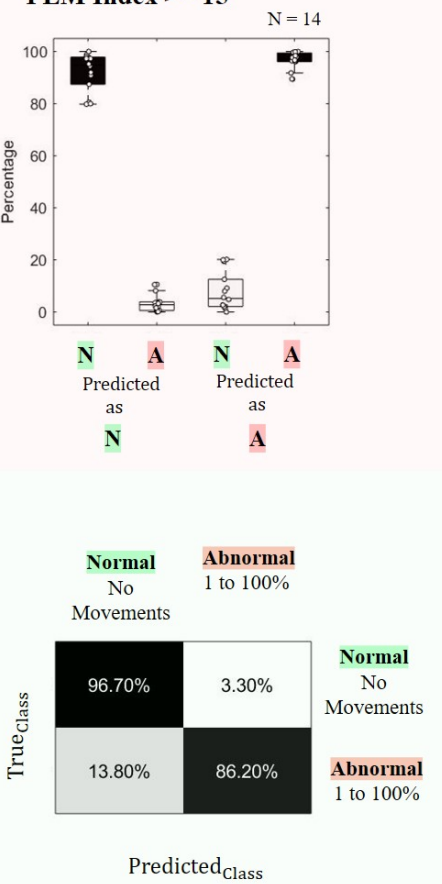

NORMAL Group

PLM Index $<15$

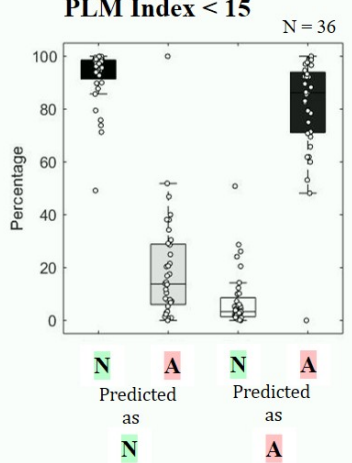

NOTE: Median values are shown within the confusion matrices.
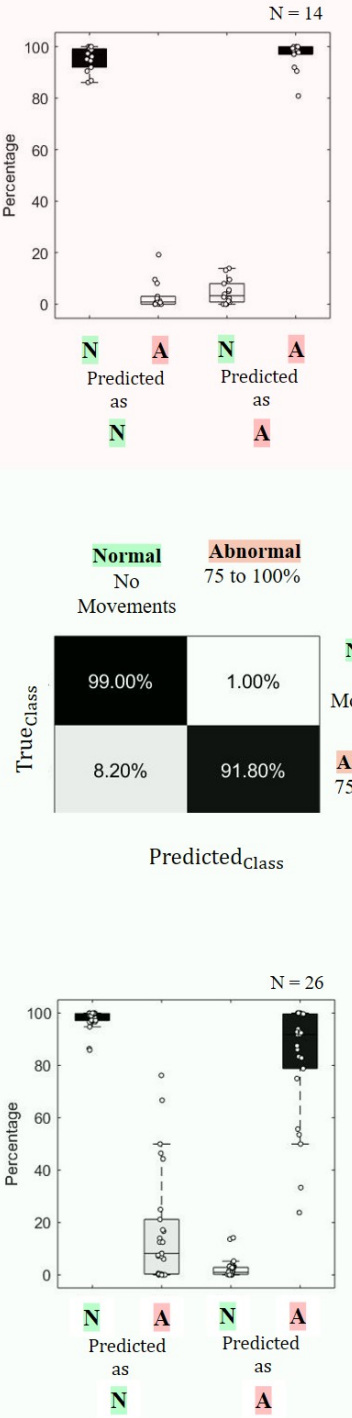

3.

Normal: No Movements

Abnormal: 75 to $100 \%$
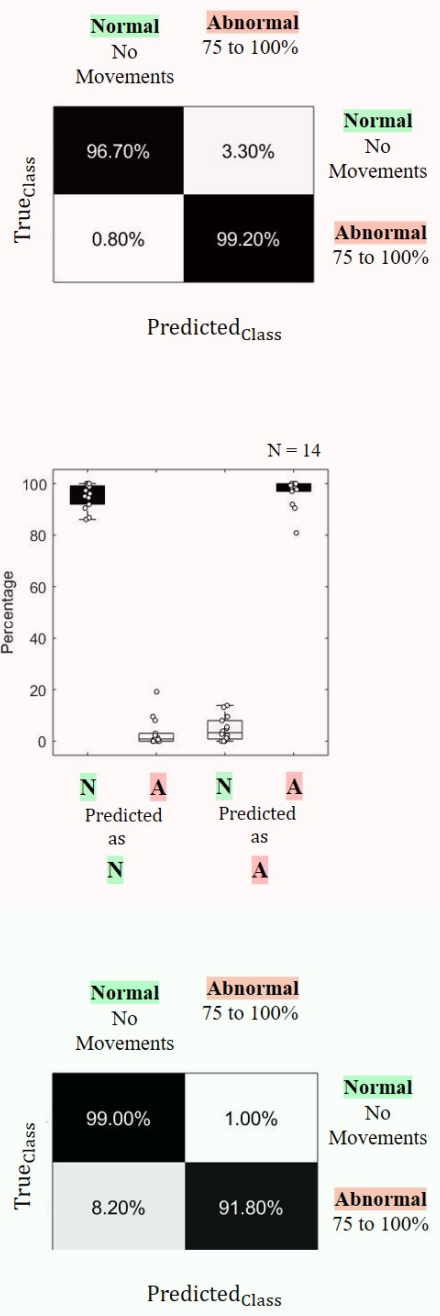

Predicted $_{\text {Class }}$

(1)

Figure 4.20: Dataset A - Classification Results on Normal and Abnormal groups of Subjects 
1.

Normal: None vs. Some Movements

Abnormal: 1 to $100 \%$
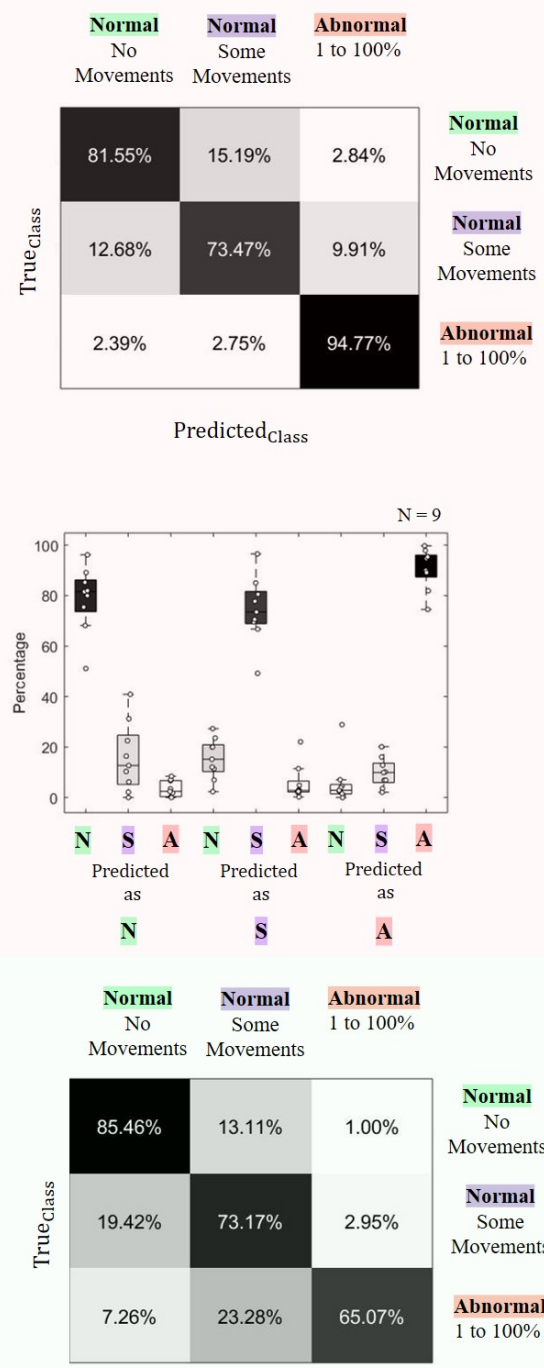

$\begin{gathered}\text { Normal } \\ \text { No } \\ \text { Movements }\end{gathered}$
Normal
Some
Movements

Abnormal
1 to $100 \%$

Predicted $_{\text {Clas }}$

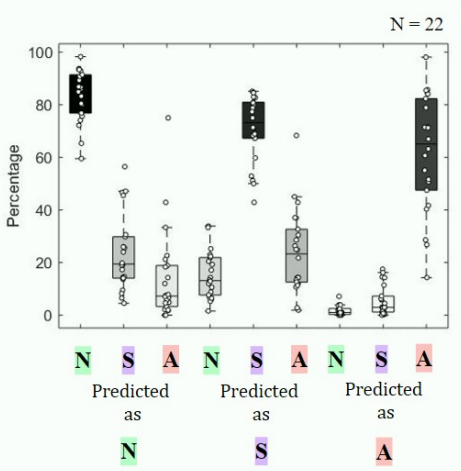

Normal: No Movements

Abnormal: 1 to $100 \%$

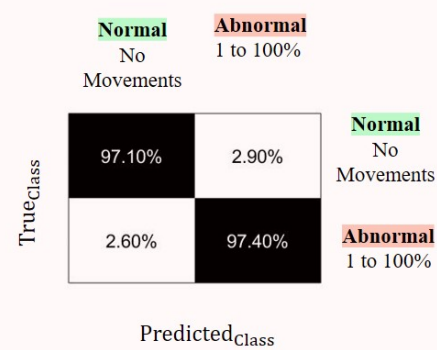

ABNORMAL Group

PLM Index $>=15$
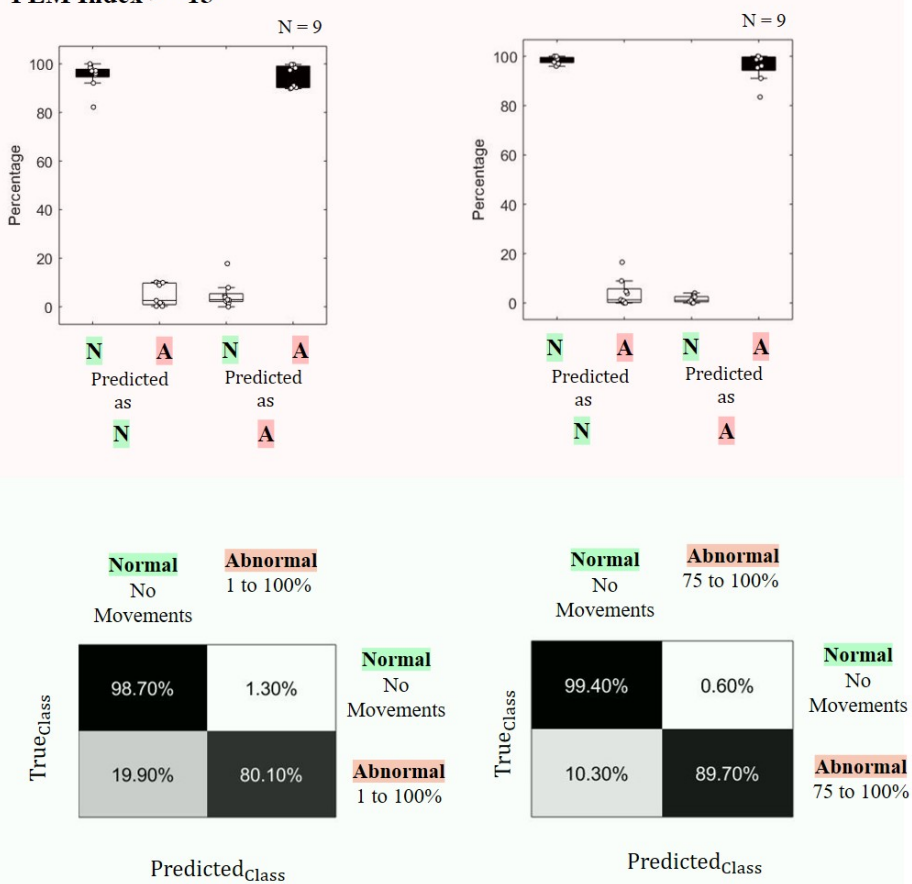

NORMAL Group

PLM Index $<15$
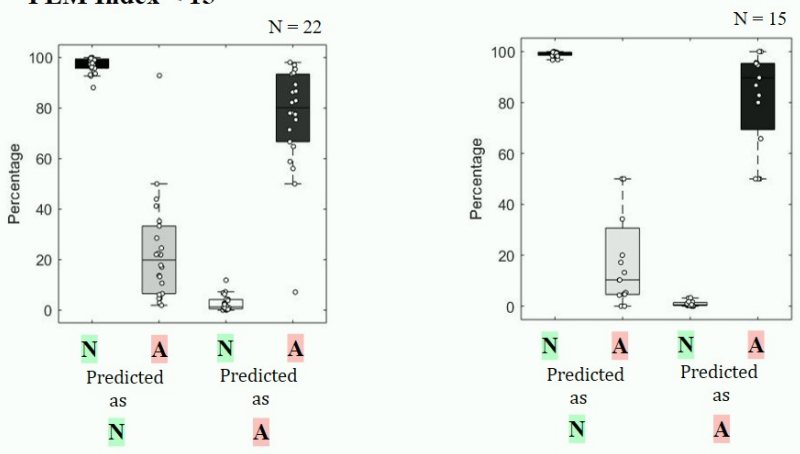

NOTE: Median values are shown within the confusion matrices.

Figure 4.21: Dataset B - Classification Results on Normal and Abnormal groups of Subjects 
the general trend in the results across the different case scenarios is first discussed. Specifically, for that of both the Datasets A and B depicted in Figures 4.20 and 4.21 respectively, in general, the median scores for the $T P_{\text {rate }}, T N_{\text {rate }}$ seemed to show an increasing trend, or in other words, the median scores for the $F P_{\text {rate }}$, and $F N_{\text {rate }}$ seemed to show a decreasing trend, across the scenarios 1 to 3 being analyzed for each group. Note though, that these trends, as discussed later, seemed to be less so for the individuals from the normal group in comparison to the individuals belonging to the abnormal group. Not only can this be made out from the median scores, but also, this can be seen from the spread of the scores (in the boxplots) for the two, i.e., normal and abnormal groups. Likewise, as depicted in Tables 4.1 and 4.2 for Datasets A and B respectively, while the single measured median MCC scores seemed to show an increasing trend going from case scenario 1 to 3 , the increase seemed to be relatively less so for the individuals from the normal groups compared to that of the individuals from the abnormal groups of either Datasets.

Table 4.1: MCC Scores of Dataset A for the 2 Groups of Subjects across the 3 Case Scenarios

\begin{tabular}{l|c|c|c}
\hline & ABNORMAL & & \\
\hline \hline & Median & 25th Percentile & 75th Percentile \\
\hline \hline 1. $(\mathrm{N}=14)$ & 0.74 & 0.69 & 0.77 \\
\hline 2. $(\mathrm{N}=14)$ & 0.91 & 0.85 & 0.94 \\
\hline 3. $(\mathrm{N}=14)$ & 0.95 & 0.90 & 0.99 \\
\hline & NORMAL & & \\
\hline \hline & Median & 25th Percentile & 75th Percentile \\
\hline \hline 1. $(\mathrm{N}=36)$ & 0.62 & 0.55 & 0.66 \\
\hline 2. $(\mathrm{N}=36)$ & 0.78 & 0.66 & 0.88 \\
\hline 3. $(\mathrm{N}=26)$ & 0.90 & 0.79 & 0.97 \\
\hline
\end{tabular}

Note that, the MCC scores can take up values within the range of -1 to 1 with 1 indicating perfect 
Table 4.2: $\mathrm{MCC}$ Scores of Dataset B

for the 2 Groups of Subjects

across the 3 Case Scenarios

\begin{tabular}{l|c|c|c}
\hline & ABNORMAL & & \\
\hline \hline & Median & 25th Percentile & 75th Percentile \\
\hline \hline 1. $(\mathrm{N}=9)$ & 0.71 & 0.69 & 0.82 \\
\hline 2. $(\mathrm{N}=9)$ & 0.88 & 0.84 & 0.92 \\
\hline 3. $(\mathrm{N}=9)$ & 0.96 & 0.91 & 0.99 \\
\hline & NORMAL & & \\
\hline \hline & Median & 25th Percentile & 75th Percentile \\
\hline \hline 1. $(\mathrm{N}=22)$ & 0.63 & 0.55 & 0.69 \\
\hline 2. $(\mathrm{N}=22)$ & 0.81 & 0.72 & 0.89 \\
\hline 3. $(\mathrm{N}=15)$ & 0.90 & 0.72 & 0.94 \\
\hline
\end{tabular}

prediction and -1 indicating otherwise [63].

\section{Analysis of classification results 'across the scenarios 1, 2 and 3'}

Nevertheless, going back to comparing the median scores across the different scenarios instead of between the groups (which would be discussed later), it seemed to be the case that, going from case scenario 1 to 2, i.e., where one included the data from ' $\mathrm{S}$ ' and the other didn't, that the distinction in the HR values per subject between the extreme 'normal' ('N') and exclusively 'abnormal' ('A - 1 to 100\%') classes seemed to become better. Firstly, this implies, as indicated in past studies [23], [53], that, aside from 'PLM' (LM from the 'A' class), there also exists HR changes during 'non-PLM' ('LM from the ' $\mathrm{S}$ ' class) that interferes with the classification of HR changes between ' $\mathrm{N}$ ' and 'A' classes per subject. Secondly, as reflected in the higher misclassification median scores of ' $\mathrm{S}$ as $\mathrm{N}$ ' or ' $\mathrm{S}$ as $\mathrm{A}$ ', it can be observed that, the HR data from the ' $\mathrm{S}$ ' can equally occur closer to the continuum of the ' $\mathrm{N}$ ' spread of HR data per subject, or 'A' spread of HR data per subject, 
respectively. Specifically, given that, only the median per segment was taken when extracting the features from the HR signals per subject earlier, and using the knowledge that non-PLM can also induce HR changes [23], [53], it is presumed that, the misclassification of ' $\mathrm{S}$ ' as ' $\mathrm{N}$ ' or ' $\mathrm{A}$ ' might depend on the number of movements per segment that existed in the respective segment in which the HR features were extracted from. For example, if there was more non-PLM movements for a short segment being analyzed from a subject, this is more likely to fit within the range of median HR values obtained for a PLM segment taken from the same subject, and vice versa, i.e., if there was less non-PLM movements for a short segment being analyzed from a subject, this is more likely to fit within the range of median HR values obtained from an absolutely normal segment taken from the same subject. Likewise, the same analogy can be said used for understanding the misclassification of ' $\mathrm{N}$ as $\mathrm{S}$ ' or, 'A as $\mathrm{S}$ ' instead. That is, given the interchangeability in the HR values from ' $\mathrm{S}$ ' with ' $\mathrm{N}$ ' or ' $\mathrm{A}$ ' (as discussed), the classifier could equally mis-learn the HR values of ' $\mathrm{N}$ ' or 'A' to belong to that of ' $\mathrm{S}$ '.

That being said, it was of interest to scrutinize here the improvement in median scores observed going from case scenario 2 to 3 , i.e., wherein, the reader is reminded that, for each subject, while case scenario 2 tried to distinguish between the HR data from extremely 'normal' ('N') vs. exclusively 'abnormal' ('A - 1 to 100\%') classes, case scenario 3 tried to distinguish between the HR data from extremely 'normal' ('N') and extremely 'abnormal' ('A - 75 to 100\%') classes. Again, it is also reminded here that the percent ranges for ' $A$ ' were chosen in this analysis to represent the proportion of time per windowed segment being analyzed that corresponded to a PLM region. Nonetheless, as indicated in the respective confusion matrices or MCC scores, i.e., for the scenarios 2 and 3 of either groups (normal and abnormal) of subjects from both the Datasets A and $\mathrm{B}$, it appears to be the case that, the improvement in scores seemed to be less so here than that of when going from case scenarios 1 to 2 . Overall, as implied by the latter, although the HR changes seemed to be more distinct for the data from the extremely abnormal vs. extremely normal than for all of the abnormal vs. extremely normal data, the improvement in classification was not as much as to the extent of when the non-PLM (from 'S' class) was removed from consideration. Moreover, unlike when going from scenarios 1 to 2 , given that certain subjects were discarded from analysis 
when going from 2 to 3 due to the absence of data from the extremely abnormal class, i.e., this was only the case when analyzing the normal groups from either datasets, it is doubtful here as to whether the improvement in scores was indeed due to distinctiveness in the extremely abnormal vs. extremely normal data, or whether it was due to the (by chance) removal of certain individuals contributing to the low scores in the classification process. Nevertheless, if the latter was not the case, i.e., as for the abnormal groups of either datasets that did not discard subjects going from case scenario 2 to 3 , then the improvement in scores, even if slight, when going from scenario 2 to 3, could be interpreted as being due to the changes in the proportion of time per windowed segment that corresponded to PLM. Specifically, given that, only the median HR values were obtained for any given windowed segment analyzed per subject from before when extracting features, the higher vs. lower proportion of time per segment that corresponded to PLM might have induced a more vs. less distinct HR value accordingly, when comparing the latter to that of the HR data obtained from the $\mathrm{N}$ regions of the subject being analyzed.

\section{Analysis of classification results between the normal and abnormal groups}

Referring back to Figures 4.20 and 4.21, note how, as mentioned earlier, the increasing trend in the $T P_{\text {rate }}, T N_{\text {rate }}$ (and MCC scores from Tables 4.1 and 4.2) across the scenarios, or decreasing trend in the $F P_{\text {rate }}, F N_{\text {rate }}$ respectively (for the $\mathrm{N}$ number of subjects from each case scenario), seemed to be less the case when comparing the median scores of the normal group with that of the abnormal group. Likewise, as can be deciphered from the spread of classification scores (within the boxplots) for certain classes, i.e., 'A as N' or 'A as A' or 'A as S', the latter seemed to suggest that the data belonging the 'A' regions for the individuals from the normal group were often misclassified. While this could perhaps be due to a difference in the distinctiveness in the HR changes between the 'A' classes of either groups of individuals (i.e., the HR changes being more distinct than that of the $\mathrm{N}$ classes for the abnormal versus normal groups of individuals), other interpretations of why there could have been higher misclassifications of the HR data from the subjects from the normal compared to the abnormal group is provided below. Note that, in particular, the 
interpretations below are specific to individuals with higher SA events, i.e., higher AHI indices, from either groups.

- Starting with individuals from the abnormal group for example, firstly, based on past studies [66] that indicate a higher co-occurrence of PLMS in individuals with SA, it can be presumed here that, for this group of individuals, there would be less SA regions occurring independent of PLM regions. Secondly, due to the fact that SA also induces similar changes in HR [67], it can be justified here that, there would be less HR changes in the normal regions occurring per subject due to SA (that is presumably less likely to occur outside of the PLM, i.e., 'A' regions). Hence the latter would result in less misrepresentation of the 'normal' regions from a given abnormal subject, and this would furthermore result in a better classification of ' $N$ ' vs. 'A' regions for the subject in question. To illustrate this, the example of the abnormal PLM subject (who had a high PLM and high SA score) shown in Figure 4.17, is used. Specifically, notice how, given that the 'N' regions from this subject were more likely to be truly normal (with no PLM or SA that induce higher HR changes), there is less chances that the classifier would mis-learn from the ' $\mathrm{N}$ ' classes from this subject. Moreover, given that the 'A' regions probably masked the SA regions in this subject, and that either of them would have had a similar effect on the $\mathrm{HR}$, it is also less likely here that the ' $\mathrm{A}$ ' regions from this subject would be misclassified. Aside from that, interestingly, as published by a recent (2019) paper, it has been found that, for individuals with PLM and SA (as was the case for the subject being analyzed here), that the HR, i.e., reflecting the autonomic system, would have a 'synergistic' effect [68]. In other words, rather than for individuals with PLM or SA alone, the HRV parameters in their study (the LF-based feature, for example) for individuals with both PLM and SA were found to be higher [68]. Hence, it isn't unexpected that the HR changes depicted in the longitudinal HR subplot (shown in Figure 4.17) for the subject here was distinct in that, the HR changes corresponding to the segmented PLM (possibly masking the SA regions) were clearly high (in the case of LFiA) or low (in the case of HFiA). 
- That being said, the opposite effect is expected for the individuals with higher SA indices from the normal group. In this case, given that there would be less PLM regions to start with for this group of individuals, it is more likely that there would be regions in the signal that would coincide with SA events occurring independent of PLM. This would, as a result, result in more 'normal' regions to have HR changes mimicking that of the abnormal regions. Again, this is provided that, similar HR changes (to that of PLM) are induced in SA regions as well [67], as mentioned earlier. Essentially, what the latter signifies is that there is more misrepresentation of ' $\mathrm{N}$ ' regions for this group of individuals in particular, and that the latter might result in higher mis-classifications overall. Specifically, this is as illustrated in Figure 4.22 which shows a normal subject with a high SA score. Specifically, note how, from the scatter plots from the 2 nd and 3rd case scenarios, it can be gathered here that some of the ' $N$ ' regions from the signal had $\mathrm{LFiA}$ and HFiA values that were similar to that of those from the 'A' regions of this subject. As a side note, note that this subject is labelled as a 'normal' individual only based on the clinical score of 0 assigned by the clinicians (who disregarded PLM associated with respiratory, i.e., SA events, based on [34]). Technically, as assigned by the algorithm (which did not disregard the PLM associated with SA events) the PLM score worked out to be 21.8), which is an 'abnormal' PLM score. Regardless, given that the SA score was extremely high, i.e., 90.4, versus (even if) the PLM score was taken into consideration, i.e., 21.8., it is highly likely that, for this individual, there would still be regions in the signal that coincided with SA events occurring independent of PLM events. Hence, it was still valid to use this subject to illustrate the concept here. As another side note, notice how there are lots of regions in the segmented PLM signal of this subject that coincide with non-PLM 'S' events. It is highlighted here that, if it wasn't for observing the signal through the perspectives of case scenarios 2 or 3 , where the HR data corresponding to non-PLM 'S' regions are discarded, the observation of the ' $\mathrm{N}$ ' regions being mixed up with the ' $A$ ' regions would not have been picked up visually (as is the case of scenario 1 depicted in the figure).

Essentially, from all of the above, it can be concluded here that, other sleep disorders (other than 

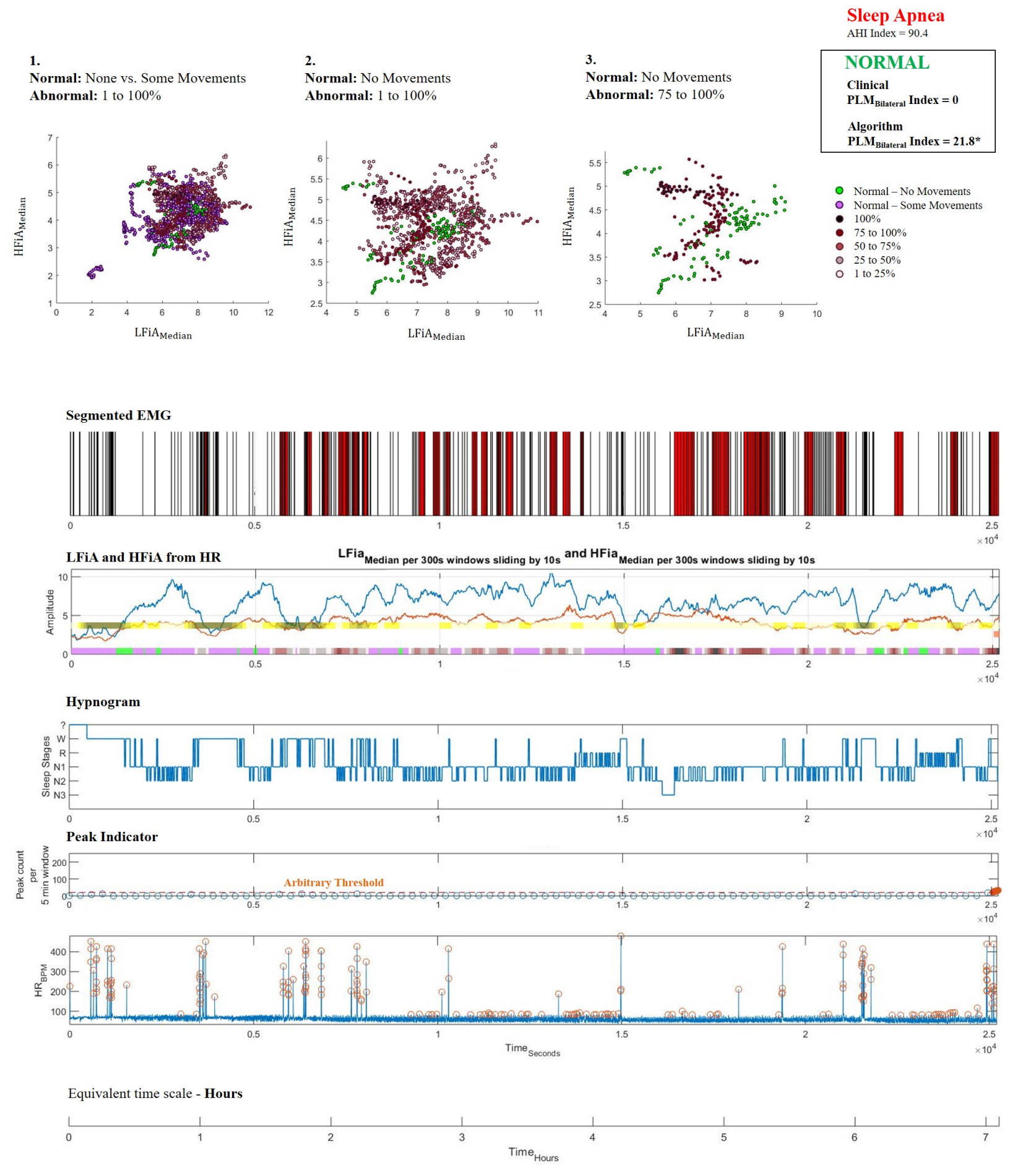

*Algorithm PLM Bilateral $_{1}$ Sndex is higher than Clinical PLM Intensity [\%]

Figure 4.22: Sample 'Normal' Subject with Sleep Apnea (and Respiratory-related PLM) 
PLM), such as that of SA for example, that possibly also induces similar HR changes [67], needs to be taken into account when monitoring for PLMS using HR. In fact, it is significant to point out here that, even in normal individuals, HR parameters differ across the different stages of sleep [69]. Hence, it is hoped that, for future work, the HR changes associated with the regions specific to other sleep disorders, that is further differentiated by the different stages of sleep, is compared with the HR changes associated with the identified PLM regions.

With that said, the practical implications asccociated with the findings from this chapter as a whole, is detailed towards the end of Chapter 5.

\subsection{Summary}

Overall, in the first half of current chapter, the steps involved in obtaining the HR information from sleep-derived ECG signals, the challenges involved in processing such signals, and the measures taken to overcome the latter, were discussed. In the second half of the chapter, relevant features were extracted from processed HR signals using the methodology adopted from [10], and the latter was used to differentiate between PLM and non-PLM periods occurring unique to a given subject (across 3 scenarios). Upon executing the methods outlined on the provided datasets, it was found that the movements within the non-PLM regions (i.e., that did not qualify to be PLM) interfered with the distinction between PLM and non-PLM regions per subject. Moreover, it was also found that the percentage of time per segmented region that corresponded to PLM resulted in more distinct HR changes per individual. Finally, comparing the percent of correctly classified segments for the individuals belonging to the abnormal versus normal groups, it was found that the scores were generally higher for the individuals from the abnormal group than that of the normal group. The possible factors for the latter, such as the co-occurrence of SA with PLMS for example, and their resulting HR changes, were scrutinized towards the end of the chapter. With that said, as mentioned earlier, the implications of the results from this chapter is elaborated in the upcoming chapter, Chapter 5. 


\section{Chapter 5}

\section{Conclusion}

\subsection{Summary, Limitations, and Future Work}

As indicated in the beginning of the thesis, i.e., in the objectives section specifically, to aid with the long-term monitoring of PLMS from all aspects possible, several research tasks were undertaken in the current thesis.

To start with, in order to overcome the time-consuming and tedious nature of manually scoring for PLM, automated PLM detection using the guidelines dictated by [7] was carried forth in chapter 3. In essence, the steps used to implement the guidelines were provided with illustrations. Following the latter, the developed algorithm was applied to the two datasets (with $\mathrm{n}=$ 56 and 38, respectively) provided to us, courtesy of Sunnybrook Hospital. Application of the algorithm resulted in the localization of PLM segments for each subject. From the detected PLM segments, the PLM Index was further calculated for each subject, as required by [7], i.e., by discarding the PLM that corresponded to the awake regions (obtained from the Hypnogram data) in the signals [7]. Moreover, in order to evaluate the performance of the developed algorithm, the scores provided by the clinicians were compared to that of the algorithm. Specifically, in order to do so, Spearman correlation, Linear regression, and Bland-Altman plots were used. However, note that, these techniques were only implemented on the subjects from the datasets who had AHI Index less than 5 ( $\mathrm{n}=28$ and 27, respectively). Specifically, this was so as to be able to provide a fairer comparison of the algorithm's scores with that of the clinicians' for which, unlike the al- 
gorithm, the PLM corresponding to the respiratory-related events were excluded, in addition to those excluded (as mentioned earlier) based on the awake regions in the signal. In other words, since the occurrence of respiratory-related events, reflected by the AHI scores, were expected to be negligible for the subjects with AHI less than 5, the performance of the algorithm was only evaluated using these subset of individuals. Regardless, the results were promising in that, for those subset of individuals for whom the Spearman correlation, Linear regression, and Bland-Altman techniques were implemented on, it was found that the scores were (fairly) strongly correlated with that of the clinical scores with correlation coefficients equal to 0.78 and 0.8 (for Datasets A and $\mathrm{B}$ ), the gradient and $\mathrm{y}$-intercepts of the linear regressions were close to being one-to-one, i.e., $0.82 x+1.82$ and $0.88 x+1.57$ (for Datasets $A$ and B), and the absolute differences between the two sets (algorithm and clinical) of scores for a majority of the individuals were less than 10 (for either datasets), respectively. However, since the algorithm currently did not exclude PLM corresponding to respiratory-related events, one of the limitations in the current study was that the performance of the algorithm could not be evaluated on the subjects with AHI greater than or equal to 5 from the provided datasets (though speculations were still provided). That being said, for future work, it is expected that such subjects would be included in the performance evaluations by discarding the PLM events corresponding to respiratory-related events based on the specifications for respiratoryrelated events by [34] (for example). On the other hand, it is speculated that one other limitation of the current algorithm was that for a few individuals $(n=2)$, that are excluded from the summary of results provided above, the number of PLM per subject were either highly under-estimated, or, over-estimated, possibly due to dynamic changes in the baseline of the signals. Hence, for future work, it is expected that a systemic methodology is adopted to deal with such issues.

That being said, with regards to the analysis conducted on PLM incidence and sleep stages, it was found that PLM incidence was found to be significantly higher $(\mathrm{p}<0.05)$ during sleep than the awake stages of sleep. Moreover, within sleep itself, PLM incidence was found to be significantly higher $(\mathrm{p}<0.05)$ during the non-REM stages of sleep, i.e., specifically during non-REM 1 and 2, compared to the other stages of sleep. Although the first finding was somewhat different from past studies, i.e., wherein PLM was more likely to be found during awake than sleep, the second 
finding, with regards to finding more PLM during the non-REM stages of sleep compared to the other stages of sleep, was consistent with past findings [28], [30], [43]. Nonetheless, as speculated earlier in section 3.3.2 of chapter 3, given that the difference in the results for the former could be attributed to be due to the difference in the subset of subjects used in the analysis, it is of interest to reiterate here that, from a monitoring perspective, this kind of analysis helps in localizing the regions in a long-term signal wherein PLM are most likely to occur, i.e., based on the various stages of sleep.

Along the same lines, by delving further in depth into the characteristics of the LM and IMI durations of the PLM found across the different stages of sleep, it was found that the PLM were likely to be shorter in duration with a widespread IMI during the REM stage of sleep, and vice versa, i.e., longer in duration with a shorter IMI during the non-REM stages of sleep, i.e., nonREM stages 1 and 2, in particular. In short, analogous to past studies (for e.g., [43]), it was postulated from the findings here that, while the mechanisms underlying the REM stages of sleep possibly inhibited the occurrence of PLM, the mechanisms underlying the non-REM stages, i. e., specifically non-REM 1 and 2, most likely facilitated the occurrence of PLM [43]. However, the results for the LM occurring within the awake stage of sleep were inconclusive. Nevertheless, as concluded earlier, it is reiterated here that larger more representative datasets are needed to affirm the speculations made here. Specifically, it is expected that the results from the latter would aid with providing us with some much needed insights on the PLM phenomenon, as a whole.

That being said, with regards to the relationship between HR and PLM, from a physiological standpoint, the use of HR signals as an alternative signal to EMG signals (obtained from PSG) to monitor PLM was discussed in chapter 2. Specifically, from the latter [15], and past studies (for e.g., [16]), it was deduced that PLM were more often than not associated with an enhanced sympathetic system activity which, incidentally, is reflected by parameters such as those obtained from the LF and HF components of HRV. In essence, as mentioned at the beginning of the thesis, the analysis in chapter 2 was carried out so as to validate the use of HR signals to monitor the progression (or management) of PLM in a given subject who, was specifically, previously diagnosed with having PLM. The reader is reminded at this point that, the purpose of all of the latter was to 
be able to avoid the issues associated with conventional PSG that, for the reasons mentioned in the beginning of the thesis, made it unsuitable for long-term monitoring of PLM.

Regardless, as presented in chapter 4, in order to implement the monitoring of PLM using alternative signals, i.e., HR signals in this case, features from the latter were extracted using the techniques specified in [10]. In particular, the two core features believed to be the most reflective of the autonomic system [22], i.e., those pertaining to the LF and HF components of the HR signals, were chosen, and further extracted, from each of the subjects belonging to the Datasets A and B. To summarize, the sequence of steps used to extract these features involved bandpass filtering the HR signals into LF and HF signals, applying HT to those signals, acquiring the instantaneous amplitudes at each point in time from the respective analytical signals (obtained through applying HT on the LF and HF signals), and finally, taking the median per 5 minute window sliding (by 10s) across the (two) analytical signals [10]. Following the latter, the identified PLM segments from chapter 3, were used, in order to train the data pertaining to the two features, i.e., the LFia $a_{\text {median }}$, and HFia median, obtained per subject. In particular, it is of interest to mention here that the 5-fold cross-validated ensemble classifier, with bagging, and the performance metrics associated with $T P_{\text {rate }}, T N_{\text {rate }}, F P_{\text {rate }}, F N_{\text {rate }}$, and MCC, were used, to deal with the presence of imbalanced data, that occurred, in (some of) the subjects being analyzed in the current study. Nevertheless, with respect to the classes pertaining to the normality and abnormality of the PLM regions, the 'N', 'S', 'A-1 to 25\%', 'A-25 to 50\%', 'A-50 to 75\%', 'A-75 to 100\%', and 'A-100\% (inclusive)' classes were used. The reader is reminded here that, for any given subject, while the first two classes represented HR segments that corresponded to 'no movements' and 'non-PLM' movements in the subject, respectively, the latter five represented the HR segments that, for ' 1 to 25 ', ' 25 to 50', '50 to 75', '75 to 100', and '100' percent of time per HR segment, corresponded to the 'PLM' segments in the subject, respectively. Moreover, somewhat similar to the PLM scoring concept, in which the PLM segments corresponding to the awake regions in the hypnogram are discarded [7], here as well, the HR segments per subject, that corresponded to ' 1 to 75 ' percent of time per HR segment to the awake regions of the hypnogram data, were discarded. Last, but not least, regions in the HR signal per subject that corresponded to signal loss, as well as possible noise (indicated 
by the peak indicator used in the current thesis), were not used in the analyses that followed.

Nevertheless, by creating three case scenarios, i.e., wherein, the data from 1), 'N' vs ' $S$ ' vs all the 'A' segments, 2), 'N' vs all the 'A' segments, and 3), 'N' vs the 'A-75 to $100 \%$ (inclusive)' segments, per subject, were studied independently, it was inferred overall (from the selected performance metrics) that, 1), the HR pertaining to the non-PLM, i.e., ' $S$ ' regions, interfered with the classification between the ' $N$ ' and 'A' classes per subject, 2), that otherwise, the classification between the ' $N$ ' and 'A' classes were distinct per subject and 3), that the latter was even more distinct when classifying between the ' $\mathrm{N}$ ' and extremely abnormal ('A-75 to 100\%') classes, respectively. That being said, although the results in the analysis seemed promising in that, given the HR values, a personalized mapping of the PLM vs non-PLM regions per subject could be obtained from learned instances, the HR changes, somewhat present at varying levels corresponding to the nonPLM (or, 'S' regions) per subject (which is consistent with [23], for e.g.), is a factor that cannot be neglected when monitoring for PLMS. Moreover, given that the HR changes associated with the 5 min segments that fully corresponded to the PLM regions per subject differed from those that only partially corresponded to the PLM regions per subject, it was further concluded that, if the HR changes corresponding to PLMS were to be monitored using the techniques by [10] adopted here, then the HR changes, based on the percent of time per HR segment that corresponded to the PLM segments in the subject, should be discriminated (for the data belonging to the low or highly concentrated regions of PLM per subject, at least). Also, aside from the latter, it was also pointed out in chapter 4 that, sleep disorders, such as sleep apnea, that possibly also induced similar HR changes [67], additionally needs to be considered when going forth with realizing the methods [10] discussed here. This was evident when comparing the results from the analyses made from the normal subset of subjects (PLM index < 15) to that of the abnormal subset of subjects (PLM index $\geq 15$ ) from either datasets. Specifically, given that the results for the normal group were somewhat slightly lower compared to that of the abnormal group, it was interpreted towards the end of chapter 4 that this could have been due to a higher likelihood of mis-learned instances of the 'N' class per subject, i.e., being masked by the sleep apnea regions in the subject. Specifically, this was construed to be the case for the subjects who had higher AHI indices compared to 
PLM indices, the latter being more likely the case for the individuals from the normal group than that of the abnormal group. That being said, apart from all that, as pointed out in Chapter 4, the fact that there exists a difference in the HR parameters between the different stages of sleep, even in normal individuals [69], though not tested here, leads us to conclude that, future studies, with region-specific labels of the occurrence of the different sleep disorders that induce HR changes (in addition to PLMS), that is further differentiated by the HR parameters obtained through the different stages of sleep, needs to be simultaneously conducted, before affirming whether the proposed method (adopted from [10]), is suitable to monitor PLMS from HR parameters on an individual basis across subsequent sessions.

In essence, given that factors such as the presence of non-PLM, region-specific incidence of co-morbid sleep disorders such as sleep apnea [67], or that even normal sleep [69], could in general, induce HR changes, rather than predicting the presence of PLM from HR, a more immediate application of the proposed procedure (adopted from [10]) would be to monitor, from the incidence of PLM, the HR changes reflecting the autonomic health of the individual. Specifically, given that a one-to-one mapping of PLM-affected regions versus HR was accomplished in the current study, the idea here would be to monitor the HR changes associated with PLM over time. In essence, as mentioned at the beginning of the thesis, it is anticipated that the latter would serve as viable markers to track, for the individuals severely affected with PLM for example, the changes in their HR parameters, in response to the medications used to treat PLM. Specifically, provided that the link between PLM and CVD exists (for e.g. [4]), the latter is in line with studies that test for whether treating PLM for example, helps with reducing the risk of acquiring CVD (for e.g., [12]). Nevertheless, it is hoped that, with the help of longitudinal studies, i.e., studies in which an individual is followed across subsequent sessions, that the above could be verified.

\subsection{Conference Proceeding from the thesis}

S. Kolappan et al, "A low-cost approach for wide-spread screening of periodic leg movements related to sleep disorders," in 2017, . DOI: 10.1109/IHTC.2017.8058167. 


\section{Bibliography}

[1] American Academy of Sleep Medicine . International Classification of Sleep Disorders . 3rd ed. Darien, IL : American Academy of Sleep Medicine ; 2014

[2] M. Alessandria, F. Provini, "Periodic limb movements during sleep: a new sleep-related cardiovascular risk factor?," Frontiers in Neurology, vol. 4, pp 1-6, 2013

[3] A. S. Walters, D. B. Rye, "Review of the Relationship of Restless Legs Syndrome and Periodic Limb Movements in Sleep to Hypertension, Heart Disease, and Stroke," SLEEP, vol. 32, No. 5, 2009.

[4] P. Congiu et al, "Periodic Leg Movements During Sleep and Cardiovascular and/or Cerebrovascular Morbidity," Current Sleep Medicine Reports, vol. 4, (1), pp. 58-64, 2018.

[5] The top 10 causes of death, 2014, http://www.who.int/mediacentre/ factsheets/fs310/en/[Accessed online - 7th December 2016].

[6] Polysomnography - Sleep Apnea, http://bozwell.co.uk/poly.html [Accessed online - 7th December 2016].

[7] R. Ferri et al., "World Association of Sleep Medicine (WASM) 2016 standards for recording and scoring leg movements in polysomnograms developed by a joint task force from the International and the European Restless Legs Syndrome Study Groups (IRLSSG and EURLSSG)”, Sleep Medicine, vol. 26, pp. 86-95, 2016. 
[8] Ontario, CA: Sleep Study (Polysomnography) Cost Comparison, https://www. newchoicehealth.com/places/california/ontario/sleep-study/ sleep-study-polysomnography [Accessedonline-7thDecember2016].

[9] S. S. Lobodzinski, "ECG patch monitors for assessment of cardiac rhythm abnormalities," Progress in Cardiovascular Diseases, vol. 56, (2), pp. 224, 2013.

[10] W. von Rosenberg, T. Chanwimalueang, T. Adjei, U. Jaffer, V. Goverdovsky and D. Mandic, "Resolving Ambiguities in the LF/HF Ratio: LF-HF Scatter Plots for the Categorization of Mental and Physical Stress from HRV”, Frontiers in Physiology, vol. 8, 2017. Available: 10.3389/fphys.2017.00360.

[11] S. Nannapaneni and K. Ramar, "Periodic limb movements during sleep and their effect on the cardiovascular system: is there a final answer?" Sleep Medicine, vol. 15, (4), pp. 379-384, 2014.

[12] M. Manconi et al, "Effects of acute dopamine-agonist treatment in restless legs syndrome on heart rate variability during sleep," Sleep Medicine, vol. 12, (1), pp. 47-55, 2011

[13] VanPutte., C. VanPutte, A. Russo and J. Regan, Seeley's Anatomy \& Physiology. New York: McGraw-Hill Education, 2016.

[14] Blausen.com staff (2014). "Medical gallery of Blausen Medical 2014". WikiJournal of Medicine 1 (2). DOI:10.15347/wjm/2014.010. ISSN 2002-4436.

[15] S. Clemens, D. Rye and S. Hochman, "Restless legs syndrome: Revisiting the dopamine hypothesis from the spinal cord perspective", Neurology, vol. 67, no. 1, pp. 125-130, 2006. Available: 10.1212/01.wnl.0000223316.53428.c9.

[16] T. Sasai, M. Matsuura and Y. Inoue, "Change in heart rate variability precedes the occurrence of periodic leg movements during sleep: an observational study," BMC Neurology, vol. 13, (1), pp. 139-139, 2013. 
[17] P. Hamilton-Stubbs and A. S. Walters, "Periodic limb movements," in Anonymous pp. 444449.

[18] S. Clemens, "Conversion of the Modulatory Actions of Dopamine on Spinal Reflexes from Depression to Facilitation in D3 Receptor Knock-Out Mice”, Journal of Neuroscience, vol. 24, no. 50, pp. 11337-11345, 2004. Available: 10.1523/jneurosci.3698-04.2004.

[19] M. H. Pennestri et al, "Nocturnal blood pressure changes in patients with restless legs syndrome," Neurology, vol. 68, (15), pp. 1213-1218, 2007.

[20] W. Rong, K. M. Spyer and G. Burnstock, "Activation and sensitisation of low and high threshold afferent fibres mediated by P2X receptors in the mouse urinary bladder," The Journal of Physiology, vol. 541, (2), pp. 591-600, 2002.

[21] E. Sforza, C. Juony and V. Ibanez, 'Time-dependent variation in cerebral and autonomic activity during periodic leg movements in sleep: implications for arousal mechanisms," Clinical Neurophysiology, vol. 113, (6), pp. 883-891, 2002.

[22] T. Electrophysiology, "Heart Rate Variability", Circulation, vol. 93, no. 5, pp. 1043-1065, 1996. Available: 10.1161/01.cir.93.5.1043.

[23] A. Guggisberg, C. Hess and J. Mathis, "The Significance of the Sympathetic Nervous System in the Pathophysiology of Periodic Leg Movements in Sleep", Sleep, vol. 30, no. 6, pp. 755766, 2007. Available: 10.1093/sleep/30.6.755.

[24] E. Sforza, V. Pichot, J. Barthelemy, J. Haba-Rubio and F. Roche, "Cardiovascular variability during periodic leg movements: a spectral analysis approach", Clinical Neurophysiology, vol. 116, no. 5, pp. 1096-1104, 2005. Available: 10.1016/j.clinph.2004.12.018.

[25] J. Palma et al, "Basal cardiac autonomic tone is normal in patients with periodic leg movements during sleep," Journal of Neural Transmission, vol. 121, (4), pp. 385-390, 2014. 
[26] G. Weinreich, S. Terjung, Y. Wang, S. Werther, A. Zaffaroni and H. Teschler, "Validation of a non-contact screening device for the combination of sleep-disordered breathing and periodic limb movements in sleep", Sleep and Breathing, vol. 22, no. 1, pp. 131-138, 2017.

[27] M. Cesari, J. Christensen, P. Jennum and H. Sorensen, "Probabilistic Data-Driven Method for Limb Movement Detection during Sleep", 2018 40th Annual International Conference of the IEEE Engineering in Medicine and Biology Society (EMBC), 2018.

[28] A. Stefani, A. Heidbreder, H. Hackner and B. Högl, "Validation of a leg movements count and periodic leg movements analysis in a custom polysomnography system", BMC Neurology, vol. 17, no. $1,2017$.

[29] A. Huang, P. Skeba, M. Yang, F. Sgambati, C. Earley and R. Allen, "MATPLM1, A MATLAB script for scoring of periodic limb movements: preliminary validation with visual scoring", Sleep Medicine, vol. 16, no. 12, pp. 1541-1549, 2015.

[30] H. Moore et al., "Design and Validation of a Periodic Leg Movement Detector", PLoS ONE, vol. 9, no. 12, p. e114565, 2014.

[31] Y. Athavale et al., "Actigraphic detection of periodic limb movements: development and validation of a potential device-independent algorithm. A proof of concept study", Sleep, 2019.

[32] K. Kayed, S. Roberts and W. L. Davies, "Computer detection and analysis of periodic movements in sleep," Sleep, vol. 13, (3), pp. 253-261, 1990.

[33] MATLAB 2018a. Natick, Massachusetts: The MathWorks Inc., 2018.

[34] C. Iber, S. Ancoli-Israel, A. Chesson, and S. F. Quan, "The AASM Manual for the Scoring of Sleep and Associated Events: Rules, Terminology and Technical Specifications", 1st edition, Westchester, IL: American Academy of Sleep Medicine, pp. 17-49, 2007.

[35] J. ZAR, "Spearman Rank Correlation," Encyclopedia of Biostatistics, vol. 7, pp. 5095-5101, 2005. 
[36] P. Schober, C. Boer and L. A. Schwarte, "Correlation Coefficients: Appropriate Use and Interpretation," Anesthesia \& Analgesia, vol. 126, (5), pp. 1763-1768, 2018.

[37] M. Abdullah, ”On a Robust Correlation Coefficient”, The Statistician, vol. 39, no. 4, p. 455, 1990. Available: $10.2307 / 2349088$.

[38] D. Giavarina, "Understanding Bland Altman analysis", 2019.

[39] "Polynomial curve fitting - MATLAB polyfit", Mathworks.com, 2019. [Online]. Available: https://www.mathworks.com/help/matlab/ref/polyfit.html. [Accessed:12-Aug-2019].

[40] J. Martin Bland and D. Altman, "STATISTICAL METHODS FOR ASSESSING AGREEMENT BETWEEN TWO METHODS OF CLINICAL MEASUREMENT”, The Lancet, vol. 327, no. 8476, pp. 307-310, 1986.

[41] K. Ho, J. R. Naugher, "Outlier lies: An illustrative example of identifying outliers and applying robust models", Multiple Linear Regression Viewpoints, vol. 26, no. 2, pp. 2-6, 2000.

[42] R. K. Malhotra and A. Y. Avidan, "Sleep stages and scoring technique," in, Second ed. Anonymous 2014, pp. 77-99.

[43] T. Pollmächer and H. Schulz, "Periodic leg movements (PLM): their relationship to sleep stages," Sleep, vol. 16, (6), pp. 572-577, 1993.

[44] D. Knights, E. Costello and R. Knight, "Supervised classification of human microbiota", FEMS Microbiology Reviews, vol. 35, no. 2, pp. 343-359, 2011. Available: 10.1111/j.15746976.2010.00251.x.

[45] J. Pan and W. Tompkins, "A Real-Time QRS Detection Algorithm", IEEE Transactions on Biomedical Engineering, vol. -32, no. 3, pp. 230-236, 1985. Available: 10.1109/tbme.1985.325532. 
[46] Sedghamiz. H, "Matlab Implementation of Pan Tompkins ECG QRS detector.", March 2014. https://www.researchgate.net/publication/313673153_ Matlab_Implementation_of_Pan_Tompkins_ECG_QRS_detect

[47] "ECG Primer: Calculations", Courses.kcumb.edu, 2019. [Online]. Available: https://courses.kcumb.edu/physio/ecg\%20primer/normecgcalcs. htm. [Accessed:12-Aug-2019].

[48] D. Nabil and F. Bereksi Reguig, "Ectopic beats detection and correction methods: A review", Biomedical Signal Processing and Control, vol. 18, pp. 228-244, 2015. Available: 10.1016/j.bspc.2015.01.008.

[49] F. Shaffer, R. McCraty and C. Zerr, "A healthy heart is not a metronome: an integrative review of the heart's anatomy and heart rate variability", Frontiers in Psychology, vol. 5, 2014. Available: $10.3389 /$ fpsyg.2014.01040.

[50] T. J. Sullivan, S. R. Deiss and G. Cauwenberghs, "A low-noise, non-contact EEG/ECG sensor," in 2007, . DOI: 10.1109/BIOCAS.2007.4463332.

[51] "Find local maxima - MATLAB findpeaks", Mathworks.com, 2019. [Online]. Available: https://www.mathworks.com/help/signal/ref/findpeaks.html. [Accessed:12-Aug-2019].

[52] K. C. Bilchick et al, "Prognostic value of heart rate variability in chronic congestive heart failure (Veterans Affairs' Survival Trial of Antiarrhythmic Therapy in Congestive Heart Failure)," The American Journal of Cardiology, vol. 90, (1), pp. 24-28, 2002.

[53] M. Kim, K. Cha, T. Kim, J. Jun and K. Jung, "Cardiac Activation Associated with NonPeriodic Leg Movements in Comparison to Periodic Leg Movements during Sleep in Patients with Restless Legs Syndrome and Healthy Subjects", Journal of Sleep Medicine, vol. 15, no. 2, pp. 68-73, 2018. Available: 10.13078/jsm.18015. 
[54] M. Galar, A. Fernandez, E. Barrenechea, H. Bustince and F. Herrera, ”A Review on Ensembles for the Class Imbalance Problem: Bagging-, Boosting-, and Hybrid-Based Approaches”, IEEE Transactions on Systems, Man, and Cybernetics, Part C (Applications and Reviews), vol. 42, no. 4, pp. 463-484, 2012. Available: 10.1109/tsmcc.2011.2161285.

[55] G. M. Weiss and F. Provost, "Learning When Training Data are Costly: The Effect of Class Distribution on Tree Induction,' Journal of Artificial Intelligence Research, vol. 19, pp. 315354, 2003.

[56] V. García, R. A. Mollineda and J. S. Sánchez, "On the k-NN performance in a challenging scenario of imbalance and overlapping," Pattern Analysis and Applications, vol. 11, (3), pp. 269-280, 2008.

[57] T. G. Dietterich, Ensemble methods in machine learning, In International workshop on multiple classifier systems, Berlin, Heidelberg, pp. 1-15, 2000.

[58] MATLAB Classification Learner App 2019a, The MathWorks, Inc., Natick, Massachusetts, United States.

[59] T. Wong, "Performance evaluation of classification algorithms by k-fold and leave-one-out cross validation," Pattern Recognition, vol. 48, (9), pp. 2839-2846, 2015.

[60] B. W. Matthews, "Comparison of the predicted and observed secondary structure of T4 phage lysozyme,” BBA - Protein Structure, vol. 405, (2), pp. 442-451, 1975.

[61] S. Boughorbel, F. Jarray and M. El-Anbari, "Optimal classifier for imbalanced data using Matthews Correlation Coefficient metric," PloS One, vol. 12, (6), pp. e0177678-e0177678, 2017.

[62] J. Gorodkin, "Comparing two K-category assignments by a K-category correlation coefficient," Computational Biology and Chemistry, vol. 28, (5), pp. 367-374, 2004.

[63] G. Jurman, S. Riccadonna and C. Furlanello, "A comparison of MCC and CEN error measures in multi-class prediction," PloS One, vol. 7, (8), pp. e41882-e41882, 2012. 
[64] "The RK correlation coefficient", J. Gorodkin, 2006. [Online]. Available: http://rk. $\mathrm{kvl} \cdot \mathrm{dk} / .[$ Accessed:13-Sep-2019].

[65] S. S. Khan and M. G. Madden, "A survey of recent trends in one class classification," in Anonymous Berlin, Heidelberg: Springer Berlin Heidelberg, 2010, pp. 188-197.

[66] A. Al-Alawi et al, "Prevalence, risk factors and impact on daytime sleepiness and hypertension of periodic leg movements with arousals in patients with obstructive sleep apnea," Journal of Clinical Sleep Medicine : JCSM : Official Publication of the American Academy of Sleep Medicine, vol. 2, (3), pp. 281, 2006.

[67] E. Kufoy et al, "Changes in the heart rate variability in patients with obstructive sleep apnea and its response to acute CPAP treatment," PloS One, vol. 7, (3), pp. e33769-e33769, 2012.

[68] X. Li et al., "Interaction effect of obstructive sleep apnea and periodic limb movements during sleep on heart rate variability”, Journal of Sleep Research, p. e12861, 2019. Available: $10.1111 /$ jsr.12861.

[69] R. Ferri et al, "Cyclic alternating pattern and spectral analysis of heart rate variability during normal sleep," Journal of Sleep Research, vol. 9, (1), pp. 13-18, 2000. 